\title{
GENDER ANALYSIS OF THE IMPACT OF RECENT HUMANITARIAN CRISES ON WOMEN, MEN, GIRLS, AND BOYS IN PUNTLAND STATE IN SOMALIA
}

April 2021

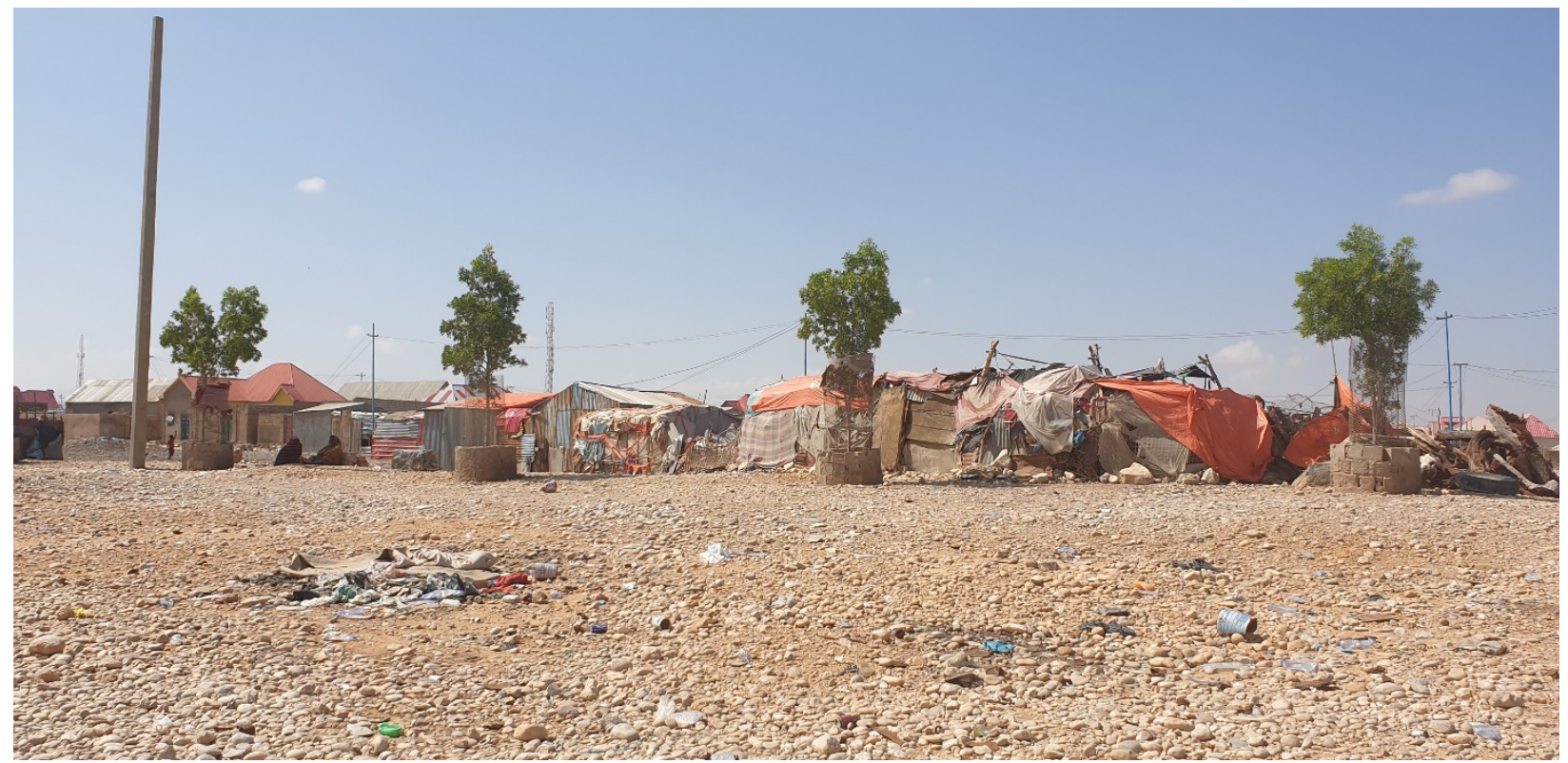

Al Khayraat IDP settlement in Garowe. Credit: Muna Hussein.

KASEY OCHILTREE, HUMANITARIAN GENDER ADVISOR (OXFAM AMERICA)

IULIA ANDREEA TOMA, GENDER ADVISOR - PROGRAMME SUPPORT TEAM (GLOBAL HUMANITARIAN TEAM) 


\section{CONTENTS}

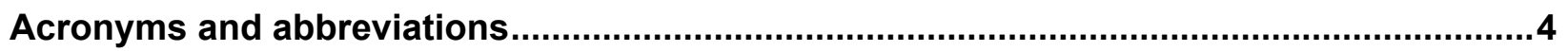

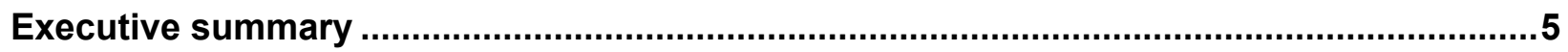

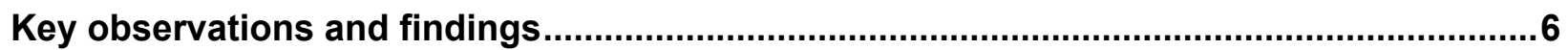

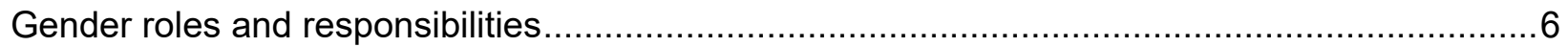

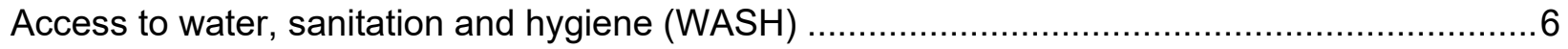

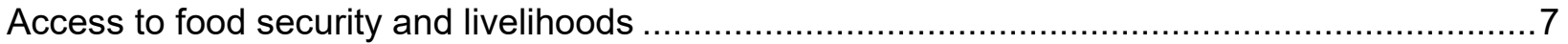

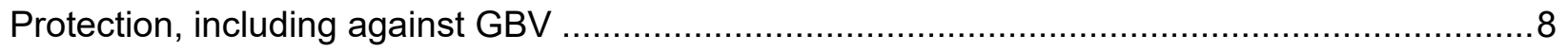

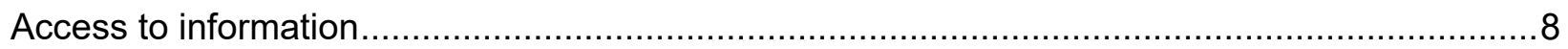

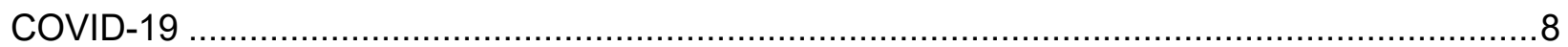

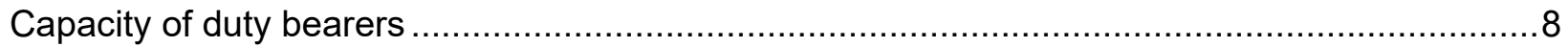

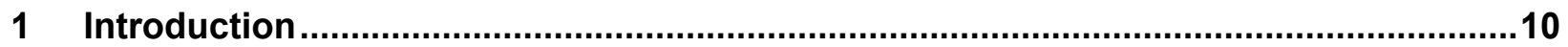

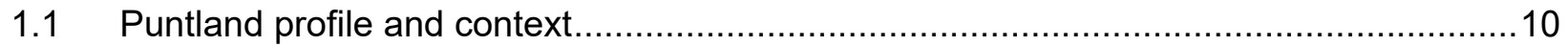

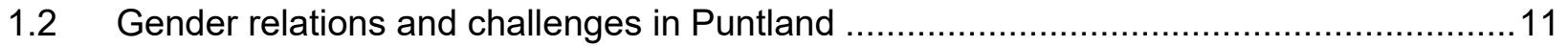

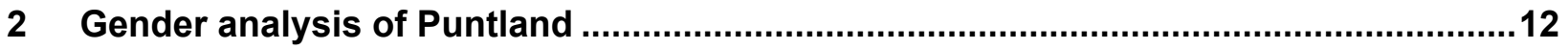

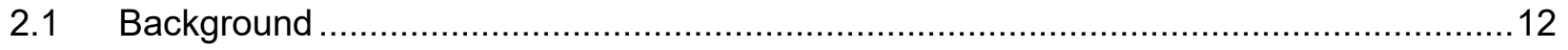

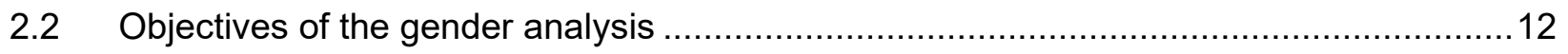

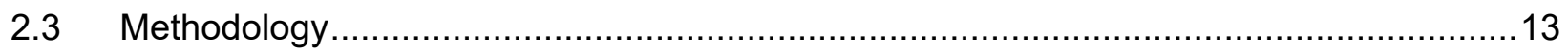

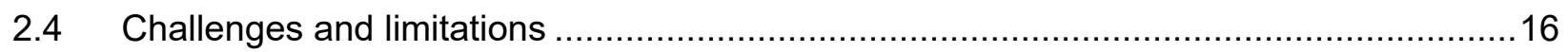

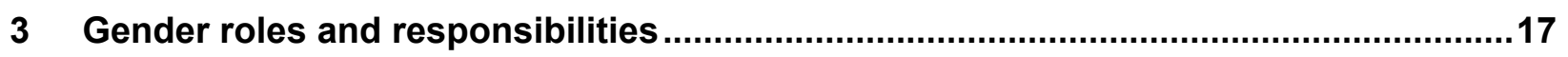

3.1 Women

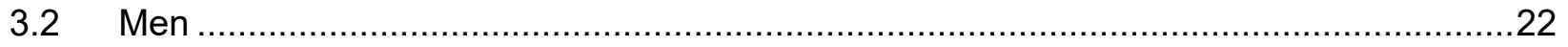

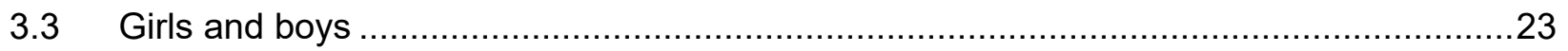

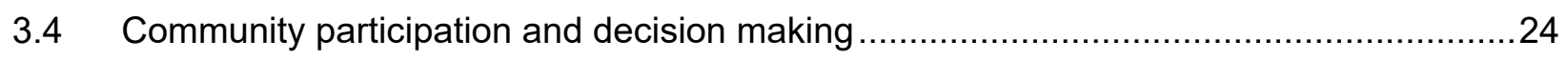

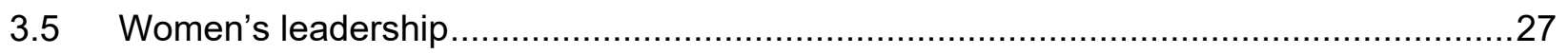

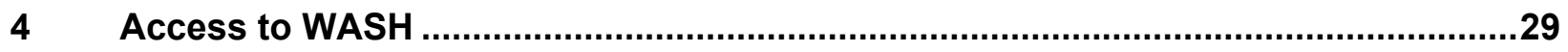

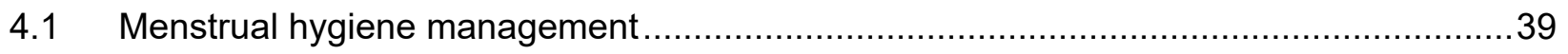

$5 \quad$ Access to food security and livelihoods ..............................................................

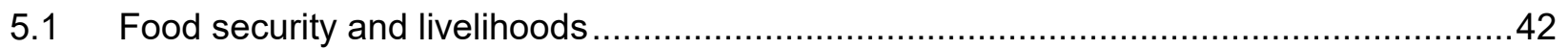




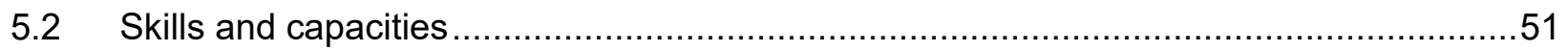

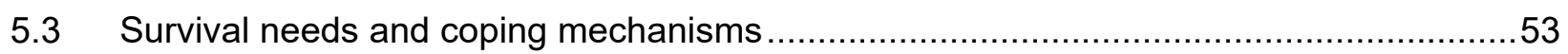

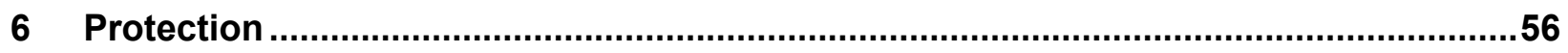

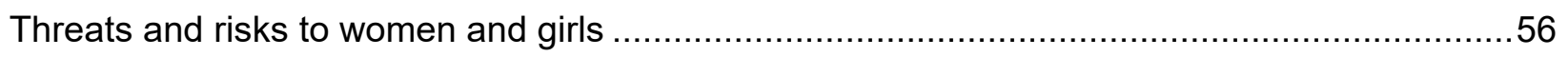

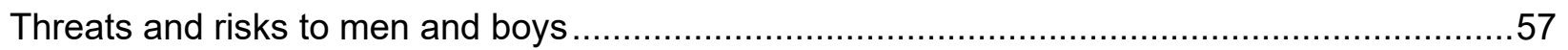

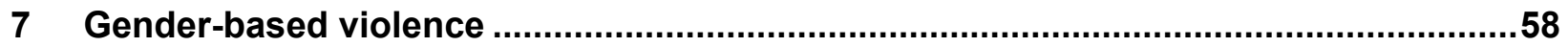

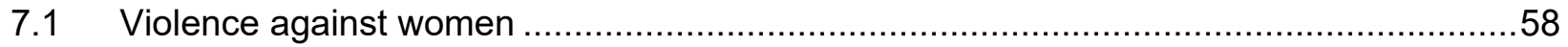

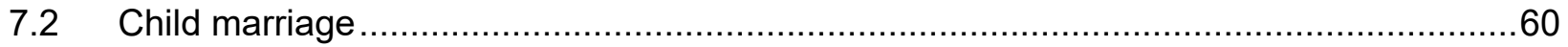

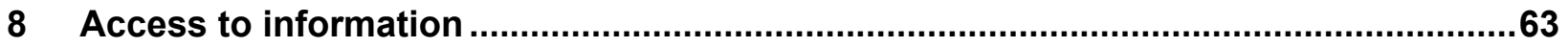

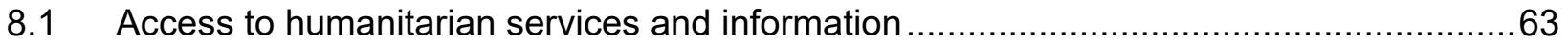

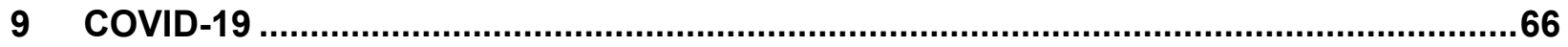

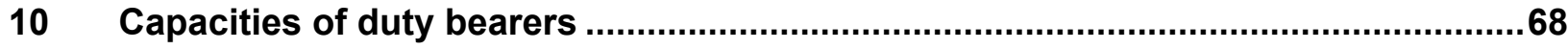

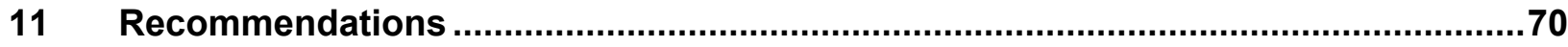

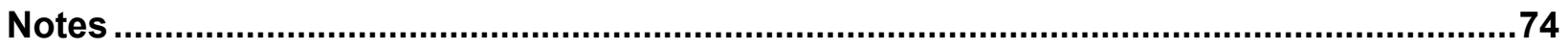

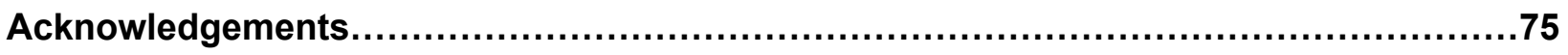




\section{ACRONYMS AND ABBREVIATIONS}

$\begin{array}{ll}\text { EFSVL } & \text { Emergency food security and vulnerable livelihoods } \\ \text { FGM/C } & \text { Female genital mutation/cutting } \\ \text { FGDs } & \text { Focus group discussions } \\ \text { GBV } & \text { Gender-based violence } \\ \text { GFFO } & \text { German Federal Foreign Office } \\ \text { IDPs } & \text { Internally displaced people } \\ \text { INGO } & \text { International non-government organization } \\ \text { IPV } & \text { Intimate partner violence } \\ \text { KAALO } & \text { KAALO Aid and Development } \\ \text { KIIS } & \text { Key informant interviews } \\ \text { MCH } & \text { Mother and child health clinics } \\ \text { NGO } & \text { Non-government organization } \\ \text { WASH } & \text { Water, sanitation and hygiene }\end{array}$




\section{EXECUTIVE SUMMARY}

Situated in a complex region of the world, Puntland State in Somalia is coping with existing threats and instabilities, such as droughts, floods, locusts, the movement of internally displaced people (IDPs), and armed actors. The COVID-19 pandemic has added yet another strain on its tremendously fragile infrastructure. The impact of the coronavirus has been far reaching, affecting livelihoods, with employment such as construction halted due to pandemic restrictions, fostering fluctuations around household assets, and hampering unpaid and underpaid care work and responsibilities. With climatic shocks and infestations, Puntland State in Somalia residents are experiencing decimated livelihoods, livestock and land, limited movement, increased violence and limited access to opportunities and support services. The multitude of crises and rates of inflation have left the majority of families food insecure and without income, halted education and health services, and exacerbated existing vulnerabilities and the incidence of violence. Needs far exceed the current available resources and capacity.

However, response efforts are under way, with many organizations and institutions adding to existing programming. Organizations such as the International Rescue Committee (IRC) have established mechanisms such as village savings and loans associations and unconditional cash transfers to help prepare for and alleviate financial shocks, while the United Nations Population Fund (UNFPA) provides vocational training and training for women's empowerment, gender-based violence (GBV) services, and women and girls' safe spaces. The ASAL ${ }^{1}$ organization works on advocacy and lobbying efforts, socioeconomic support and building awareness campaigns. KAALO Aid and Development, a local organization involved in humanitarian and development action in the area, has been working with female-headed households, through cash and in-kind support, on continued awareness of GBV prevention and response and recognizing the importance of engaging boys and men, and religious and cultural leaders as a transformative approach in reaching inclusive and gender-sensitive humanitarian programming. From the government side, the Puntland State in Somalia Humanitarian and Disaster Management Agency (HADMA) provides support to the disaster and crisis humanitarian response.

To better help local government bodies, agencies, NGOs and INGOs grasp the differentiated impact on women, men, boys and girls, and host and IDP communities during this time of intense loss and instability, this gender analysis was conducted and funded by the German Federal Foreign Office (GFFO) in partnership with Oxfam in Somalia and KAALO. The analysis provides an overview of the intricacies of the communities, with recommendations for addressing immediate concerns and future programming. It was conducted across 11 villages (in three districts - Bosaaso, Gardo and Garowe), through 34 focus group discussions (FGDs) with women, men, boys, and girls; 18 key informant interviews (KIls) from the community (of which seven were with women); and nine KIls with organizations and municipal government officials (of which two were with women). 


\section{KEY OBSERVATIONS AND FINDINGS}

\section{GENDER ROLES AND RESPONSIBILITIES}

Across all groups at the household level, women were acknowledged as the people responsible for household responsibilities, such as cleaning, childcare and cooking. Nonetheless, men remain the head of the household, though in IDP communities shifts in gender roles were noted, with women also working to provide income for the family and men helping with some household activities. In the host communities no major changes in gender roles were noted.

In terms of community participation and decision making, in IDP communities men and women reported seeing a small shift in women's participation, but with little decision-making power. Roles holding respect and power among the community such as community elders, religious leaders, and others with elite status remain the domain of men, so while women are consulted, men retain the decision-making power. The situation is similar in the host communities, though women reportedly have a little more decisionmaking power. Female leaders are rare and most work as peace promoters, on awareness raising, and as part of women's savings groups. Encouragingly, across the locations, informal and formal women's committees were recognized. Nonetheless, there are several barriers to achieving, for example, women's economic empowerment - such as traditional male beliefs that every productive job is for men and that similarly to women's leadership roles, they are too busy with housework to be able to have productive roles in the community.

\section{ACCESS TO WATER, SANITATION AND HYGIENE (WASH)}

Safe and accessible mechanisms to meet water and hygiene needs are at a bare minimum in the communities. The FGDs, KIls and survey results confirmed that potable water, secure latrines, proper lighting, accessible boreholes, and sanitary items are desired commodities for all. As there is not enough water for the communities, borehole and water reservoirs are needed. Another common issue mentioned across all communities was the lack of water cleanliness. Furthermore, the survey results, FGDs and KIIs agreed that women are the ones who often hold the responsibilities and decision-making roles related to WASH activities. In terms of safety, the communities, especially women and girls, mentioned certain activities that can put them in greater jeopardy, such as fetching water, using the toilet and bathing, especially for the IDP communities, where toilets are some distance from many of the camp inhabitants and there are no lights.

A gap in the accessibility of proper menstrual hygiene management for the women and girls in these Puntland State in Somalia communities was confirmed across the FGDs. If supplies for menstruation are available, they are costly. Private spaces for proper cleaning practices do not exist and the locations of lavatories and water sources are inconvenient. Women and girls do not have basic hygiene supplies such as soap, and resort to the use of ash and clay to clean and wash. Most of the women have access to 
pads only from humanitarian distributions, and often even these are in limited supply, with women having to resort to using cloths most of the time.

\section{ACCESS TO FOOD SECURITY AND LIVELIHOODS}

In terms of gendered livelihoods, in the IDP communities (Bulo Migis, Bariga Bosaaso, Al Khayraat, and Jowle), all the family members work in casual labour activities within the camp. Men work in fishing (in the coastal areas), pottery, building, carpentry, and other types of hard labour. Women work in cleaning services, either for households or laundry services. Some collect garbage for money while others collect empty water bottles to exchange for money or food. Women also sell products in the market such as vegetables and jewellery. Girls do similar work as their mothers, while boys work mainly in fishing (in the coastal areas) and shoe polishing. For the host communities of Kalabayr, Carta, Geri Hel, Budunbuto, Cuun, Kubo and Saacada, livestock, farming and the export of frankincense were noted as their primary sources of income. Women in Cuun collect caw, a natural material that they make mats from and then sell.

In terms of food security, coping with food shortages has become all too common and families are adjusting their consumption by reducing their daily intake, eating just once or twice a day. FGD participants mentioned that they were mainly suffering from monetary inflation. With decreases in the street value of the Somali shilling for the past year, the average person makes about $\$ 2.50-\$ 3$ per day ${ }^{2}$. The decrease in work opportunities associated with inflation has added another layer of economic strain. Furthermore, the reduction in value has resulted in the need to carry large amounts of paper money. Those doing so in public, especially at night when workers head home, regardless of their sex, are at an increased risk of theft and violence.

COVID-19 has had an impact on livelihood activities and income in both IDP and host communities. Women said that they do not receive as much work as they used to, cleaning clothes for households or collecting garbage from houses. The IDP communities highlighted the higher price of firewood, which can be tied to the rise in inflation and loss of job opportunities. For the host communities, COVID-19 lockdown measures have halted all their export activities. Women's businesses had to stop due to COVID-19 restrictions and the effect of inflation diminishing the purchasing power of nearby communities.

Many in the communities have an informal skill set that they have used to survive these hardships. As many are illiterate there is a greater focus on manual labour and informal services. Negative coping mechanisms were also reported such as eating less food, or eating fewer times a day, or selling livestock.

It is important here to note women's care burden versus decision making around emergency food security and vulnerable livelihoods (EFSVL). As observed above, women are the ones responsible for cooking, cleaning, childcare, WASH-related tasks, as well as, for some women, either supporting on or being directly responsible for bringing in income. However, all the decision making around household income and resources rests with men. So, although gender roles have shifted, especially in IDP communities, this puts women in a position where they have increased burdens as they play double gender roles, with increased demands on their time. 


\section{PROTECTION, INCLUDING AGAINST GBV}

Safety and security concerns are never-ending in precarious environments, with women and girls facing elevated risks of various forms of violence. Routine tasks such as collecting firewood or going to the market leave women and girls open to harassment, assault, or theft, particularly in IDP settings. There is a heavy dependence on the men of the family to protect the women and girls. For men and boys, the risks include kidnapping, murder, and threats from armed groups, in both host and IDP communities. Tensions around armed groups and tribal conflicts were reported as existing inside the IDP settlements.

In terms of GBV risks, domestic violence, child marriage and rape were reported across the communities, with domestic violence appearing to be the main issue in host settings, and sexual assault in IDP settings. GBV was also reported to be on the rise, specifically intimate partner violence (IPV), in IDP settings increasing due to the change in role for women as income providers, and in host settings due to financial strains. Child marriage was also reported across all locations, specifically affecting girls and at a younger age than before the crises.

\section{ACCESS TO INFORMATION}

Effective and reliable communications and information sharing in times of crisis is critical and lifesaving, especially for the most vulnerable populations. For the IDP communities, radio and hotlines seem to be the main means of sharing information, with women also receiving information from mother and child health clinics $(\mathrm{MCH})$. For the host communities, women mentioned radio, phone calls and hotlines, but they do not have the same access as men. Both communities also mentioned the need for tailored messages for each vulnerable group. Similarly, many men, women, boys, and girls were unaware of ways to engage with humanitarian organizations and access support. Key informants across locations seem to have knowledge about the humanitarian services available but there was no indication that this knowledge gets passed on to community members, especially to specific vulnerable groups.

\section{COVID-19}

The public health and security threat from COVID-19 is far reaching, and its gendered impacts are also readily apparent. There are no health services related to COVID-19 provided in the IDP and host communities, outside of awareness raising regarding the use of masks, gloves, proper handwashing techniques and social distancing. Notably, these prevention measures are less well known by women, with $45 \%$ of women saying that they did not know what they were, compared with $12 \%$ of men.

\section{CAPACITY OF DUTY BEARERS}

Out of the nine KIls with representatives of INGOs and government organizations, three INGOs had both the capacity and the resources to ensure gender mainstreaming in their projects, as well as specific responses for GBV survivors. Two of the duty bearers are best placed to provide support on the protection and GBV risks identified in this analysis, and coordination on these should be enhanced to ensure rapid responses. 
The capacity of the INGOs and government bodies regarding WASH varies, but it is clear from the community findings that more needs to be done to ensure that the needs of women, men, boys, and girls are safely and adequately met. Not all organizations systematically consult and engage with the communities. Capacities around EFSVL are also varied, with one INGO that is acutely aware of the needs and risks that different groups are encountering. All duty bearers mentioned food security as a key issue to which they respond with either training or capacity building, but that financial grants and more sustainable approaches are needed, especially given the compounded effects on food security and livelihoods of the recent crises (COVID-19, locust infestation, and drought). On protection and GBV, all duty bearers highlighted the distinct needs and vulnerabilities of the IDP communities, as well as of women and girls in particular. 


\section{INTRODUCTION}

\subsection{PUNTLAND PROFILE AND CONTEXT}

Situated in the north-east of Somalia, bordering the Indian Ocean and the Gulf of Aden, Puntland State in Somalia is a self-declared autonomous entity that remains under the federal authority of Somalia. Derived

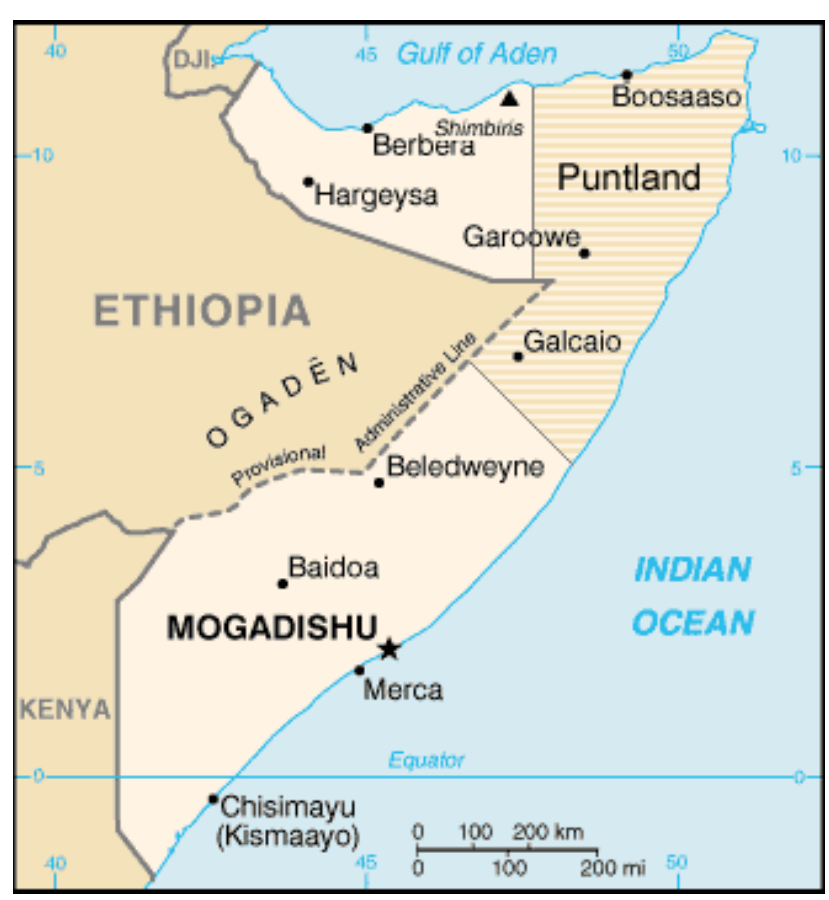

Map 1: Location of Puntland ${ }^{5}$ from the 'Land of Punt' of ancient civilizations, including early Egypt, Puntland's history is that of copious resources and as a centre of trade for the region. ${ }^{3}$

Puntland State in Somalia spans about one-third of Somalia's landmass, and has an estimated population of 4.3 million, with the densest populations living in urban areas such as Bosaaso, Galkacyo and the capital, Garowe. Livestock is the informal economic heart in the region and there is a large population of agropastoralists. However due to recent drought, it is estimated that over $60 \%$ of livestock in Puntland State in Somalia has been lost to severe dry conditions, and the surviving livestock has reduced production capacity and poor health. ${ }^{4}$

Puntland is prone to disasters, often exacerbated by droughts, floods, and conflict. For decades, the region has been the centre of contentious political upheaval, inconsistent and drastic climate variability, and protracted humanitarian crises. Less common meteorological events, such as Cyclone Gati in November 2020 , the strongest storm to hit the country in decades, ${ }^{6}$ bring further complications.

Operating as a member state of Somalia, Puntland has created its own internal degree of governance that has brought new endeavours and actors to the area. Puntland's government runs as a presidential system, with incumbent President Said Abdullahi Deni and Vice President Ahmed Elmi Karash elected to office in early $2019 .^{7}$

Regardless of political resolve to transition into a durable state of development, the population of Somalia, and Puntland State in Somalia particularly, faces many challenges that require humanitarian assistance. According to recent OCHA figures, 1.3 million people in Somalia are in a state of emergency and crisis, 2.8 million are in stress, 4.1 million are food insecure, and 2.6 million are internally displaced persons. ${ }^{8}$ The need for humanitarian aid is unremitting, causing financial strain across the sector as it tries to keep up with demand. 


\subsection{GENDER RELATIONS AND CHALLENGES IN PUNTLAND}

Though there is limited information on Puntland State specifically, information from Somalia paints a picture of harmful traditional and cultural practices such as female genital mutilation/cutting (FGM/C), lack of access to education, healthcare, and economic capital, and increasing rates of gender-based violence (GBV) that reinforce gender stereotypes and perpetuate a perilous place for women. ${ }^{9}$ The country is taking strides to mainstream gender across sectors, but it is still largely a male-dominated society and women remain heavily underrepresented in transformative dialogues and spaces. Furthermore, with a large population of internally displaced people (IDPs) and migrant populations, exposure to violence on all levels is high, even more so for women and girls. ${ }^{10}$

The 2020 Puntland Health and Demographic Survey (PLHDS), ${ }^{11}$ developed with technical support from UNFPA, provides a high-level snapshot of Puntland State in Somalia across several thematic areas. It found that:

- $31 \%$ of household heads are women;

- $33 \%$ of women and girls aged 6 and above have never been to school;

- $88 \%$ of women aged $15-49$ have no access to newspapers, radio, or television at least once a week;

- $74 \%$ of women aged $15-49$ have never attended school;

- $11 \%$ of women aged $15-19$ have either given birth or are pregnant with their first child;

- $72 \%$ of women aged $15-49$ have had at least one issue accessing healthcare;

- $32 \%$ of children under 6 months old are exclusively breastfed;

- $65 \%$ of women age $15-49$ have heard of HIV and AIDS;

- $15 \%$ of women aged $15-49$ have experienced physical violence since the age of 12 ;

- $67 \%$ of women aged $15-49$ believe that most violent acts against women take place in the home;

- $15 \%$ of ever-married women aged $15-49$ who had experienced physical or sexual violence had sought help;

- $75 \%$ of women aged $15-49$ believe that $F G M / C$ is a religious requirement;

- $79 \%$ of women aged $15-49$ want the practice of FGM/C to continue;

- $5 \%$ of household members suffer from at least one chronic disease; this proportion increases with age.

The Puntland Minister of Planning, Economic Development and International Cooperation and the Minister of Health jointly introduced the PLHDS Report 2020 with data from over 28,000 households across five regions. ${ }^{12}$ It highlighted progress and context on women's status, including that:

- for the first time, Puntland has a maternal mortality ratio (MMR) indicator (622 maternal deaths per 100,000 live births);

- health facility childbirth deliveries have increased from 13\% in 2011 to $19 \%$;

- early marriage has reduced for women married by age 18, from $38 \%$ in 2011 to $23 \%$;

- Puntland women are empowered to make financial choices - nine out of 10 women decide on how their cash earnings are spent, either individually or jointly with their husbands, and around six out of ten women of childbearing age use their phones for financial transactions. 
These are noteworthy gains in relation to women's progress in Puntland State in Somalia, and provide valuable context to this gender analysis.

\section{GENDER ANALYSIS OF PUNTLAND STATE}

\subsection{BACKGROUND}

Oxfam is one of the leading international humanitarian organizations focusing on the alleviation of global poverty and injustice through fighting inequality. Driven by diversity and commitment to the universality of human rights, Oxfam's work is feminist in its approach, guiding all its analysis, action, and interaction. Intervening in the thematic areas of water, sanitation, and hygiene (WASH), emergency food security and vulnerable livelihoods (EFSVL), and protection, with a commitment to mainstream gender throughout the programme cycle in the Nugaal and Bari regions of Puntland, Oxfam's work supports communities in coping with the compounded crises that have been affecting them in recent years.

KAALO Aid and Development is one of the most successful community based, non-profit, humanitarian and development NGOs in Somalia, ${ }^{13}$ and its headquarters are located in Garowe, Puntland. The organization was established in October 1991 just after the collapse of the Somali central government. It has a successful record in implementing more than 100 programmes and projects in both the emergency and development sectors in nine regions of Puntland. KAALO's main focus areas are in livelihoods and the productive sectors, health, education, the promotion of human rights and democracy, and WASH environmental protection. This is in line with its long-term vision and efforts to support vulnerable communities. Currently KAALO is implementing a GFFO-funded project with Oxfam. Further, KAALO implements projects that encourage women to earn and save money, such as village and saving loans associations, and also provides training on entrepreneurship and business skills. KAALO implements projects that encourage women's decision making at community and national levels through the establishment of women's forums. KAALO also advocates for the implementation of laws that guarantee access to justice and the elimination of sexual violence.

KAALO and Oxfam carried out this gender analysis under an integrated GFFO-funded project to identify the gendered needs and priorities of crises-affected communities in target communities, and to provide practical recommendations to inform programme design and implementation.

\subsection{OBJECTIVES OF THE GENDER ANALYSIS}

This analysis aims to understand the gender-related impact of multiple crises of drought, conflict, locusts, cyclones, and the COVID-19 pandemic on women, men, girls, and boys in target communities. The data collected will be used to address the gender needs highlighted in this report, and enhance current and planned KAALO programming and advocacy. 
The specific objectives of the analysis are to:

- understand the gender differences (needs, interests, capacities, roles, relations, risk, vulnerabilities) of women, men, girls, and boys and how they are affected by multiple crises of drought, conflict, locusts, cyclones, and the COVID-19 pandemic;

- understand the context and identify opportunities for increasing voice and participation of different gender groups;

- understand power dynamics, decision-making processes, and access to resources, and propose practical recommendations for the way forward;

- provide recommendations that focus KAALO and Oxfam's responses in the thematic areas of WASH, EFSVL, and protection;

- identify the capacities of duty bearers (government, international organizations, and UN agencies, international and national NGOs, and grassroots civil society organizations) in responding to the needs of affected women, girls, boys, and men by providing humanitarian services.

\subsection{METHODOLOGY}

The assessment was conducted in November/December 2020 using a desk review of secondary data as well as field data collection from primary sources, using both qualitative (focus group discussions (FGDs) and key informant interviews (KIIs)) and quantitative methods (a survey). Primary data were collected by a team composed of Oxfam and KAALO staff and 12 enumerators (six men and six women), including gender and protection staff. The enumerators were provided with training on gender, GBV, and ethical research methods during a one-day event prior to data collection.

The survey was conducted in 11 villages covering a sample size of 185 households, determined through taking a $95 \%$ confidence level and a $5 \%$ margin of error. Participatory qualitative techniques included 18 KIls with village heads and committees (of which seven were women), nine KIls with duty bearers, and 34 FGDs with women, men, boys, and girls, in line with the COVID-19 guidelines recommended by Oxfam and the government of Puntland State. Table 1 gives a detailed breakdown of locations, data collected, and numbers for the qualitative research.

Table 1: Breakdown of FGDs and KIls

\begin{tabular}{|c|c|c|c|c|c|c|c|}
\hline \multicolumn{8}{|l|}{ FGDs } \\
\hline Regions & Districts & Villages & Male & Female & Boys & Girls & Total FDGs \\
\hline \multirow[t]{7}{*}{ Bari } & \multirow[t]{4}{*}{ Bosaaso } & Bariga Bosaaso & 8 & 15 & 7 & 7 & 4 \\
\hline & & Bulo Migis & 12 & 30 & 11 & 8 & 4 \\
\hline & & Kalabayr & 15 & 13 & 8 & 8 & 4 \\
\hline & & Arta & 9 & 21 & 9 & & 3 \\
\hline & \multirow[t]{3}{*}{ Gardo } & Kubo & 13 & 15 & & & 2 \\
\hline & & Saacada & 15 & 10 & & & 2 \\
\hline & & Geri Hel & 12 & 10 & & & 2 \\
\hline Nugaal & Garowe & Cuun & 16 & 18 & & & 2 \\
\hline
\end{tabular}




\begin{tabular}{|c|c|c|c|c|c|c|c|}
\hline & & Budunbuto & 11 & 16 & & 5 & 3 \\
\hline & & Khayraat and Waberi & 12 & 18 & 13 & 25 & 4 \\
\hline & & Jowle & 9 & 18 & 10 & 8 & 4 \\
\hline Total FD & & & & & & & 34 \\
\hline KIIs & & & & & & & \\
\hline Regions & Districts & $\begin{array}{l}\text { Male } \\
\text { NGO/government } \\
\text { staff }\end{array}$ & $\begin{array}{l}\text { Female } \\
\text { NGO/UN } \\
\text { staff }\end{array}$ & Villages & Male & Female & Total \\
\hline Bari & Bosaaso & ASAL & & $\begin{array}{l}\text { Bariga } \\
\text { Bosaaso }\end{array}$ & 1 & 1 & 2 \\
\hline & & & & Bulo Migis & 2 & & 2 \\
\hline & & Municipality & & Kalabayr & 1 & 1 & 2 \\
\hline & & & & Arta & 1 & 1 & 2 \\
\hline & Gardo & & & Kubo & 1 & 1 & 2 \\
\hline & & & & Saacada & 1 & & 1 \\
\hline & & & & Geri Hel & 1 & & 1 \\
\hline Nugaal & Garowe & & Care & Cuun & 2 & 1 & 3 \\
\hline & & IRC & & Jowle & 1 & & 1 \\
\hline & & & UNFPA & Al Khayraat & & 2 & 2 \\
\hline & & ADESO $^{14}$ & & & & & \\
\hline & & KAALO & & & & & \\
\hline & & Ministry of Women & & & & & \\
\hline & & HADMA & & & & & \\
\hline Total KIIs & & 7 & 2 & & 11 & 7 & 27 \\
\hline
\end{tabular}

Seventy-four percent of survey respondents were women (Figure 1), 35\% were from the host community, $32 \%$ from the IDP community and 33\% were returnees (see Figure 2 for the gender breakdown); $42 \%$ of all respondents were from a rural area (gendered breakdown in Figure 3).

\section{Figure 1: Survey respondents' sex}

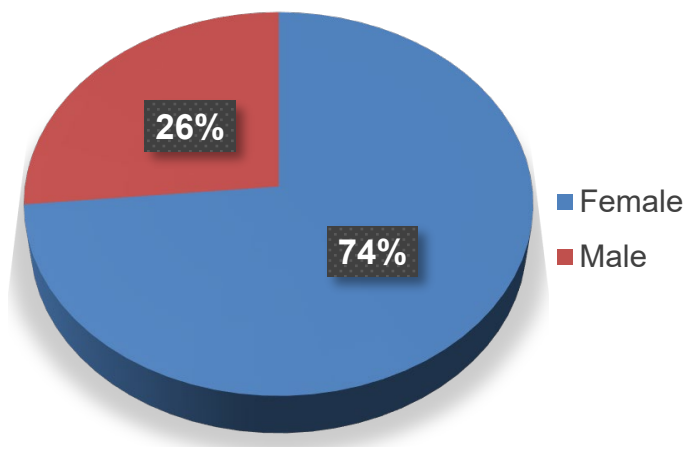


Figure 2: Survey respondents' household status

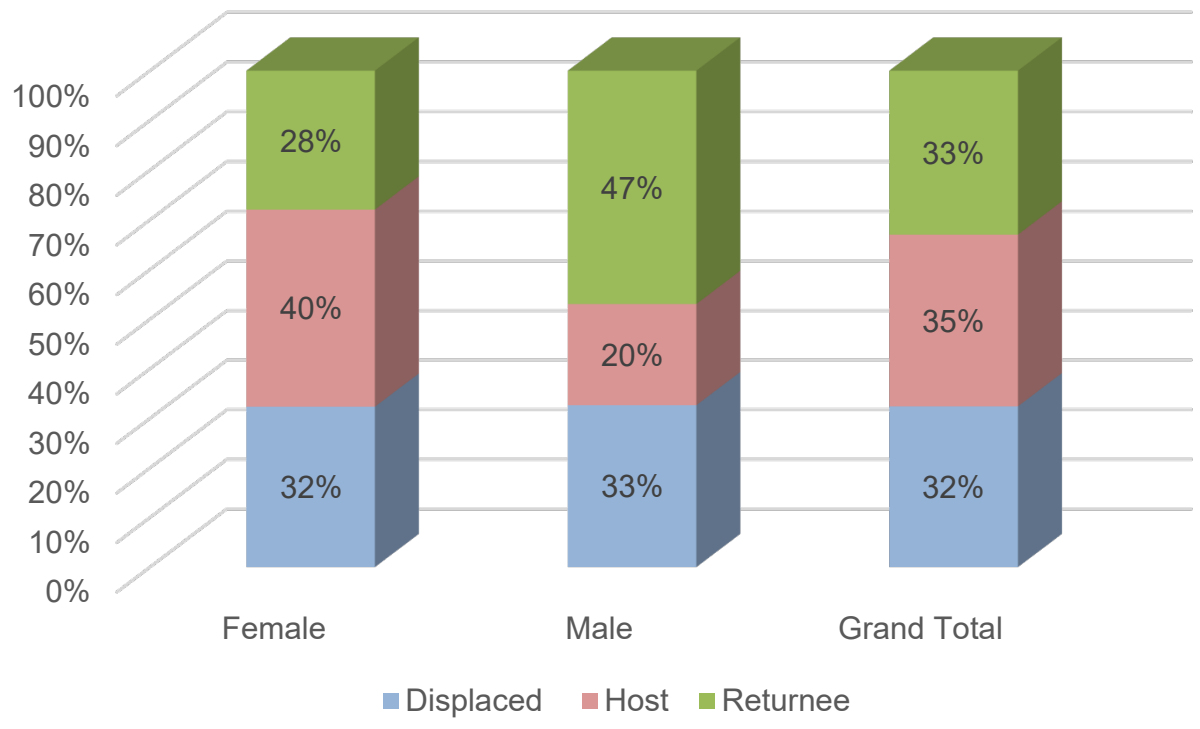

Figure 3: Household locations

Where is your household situated?

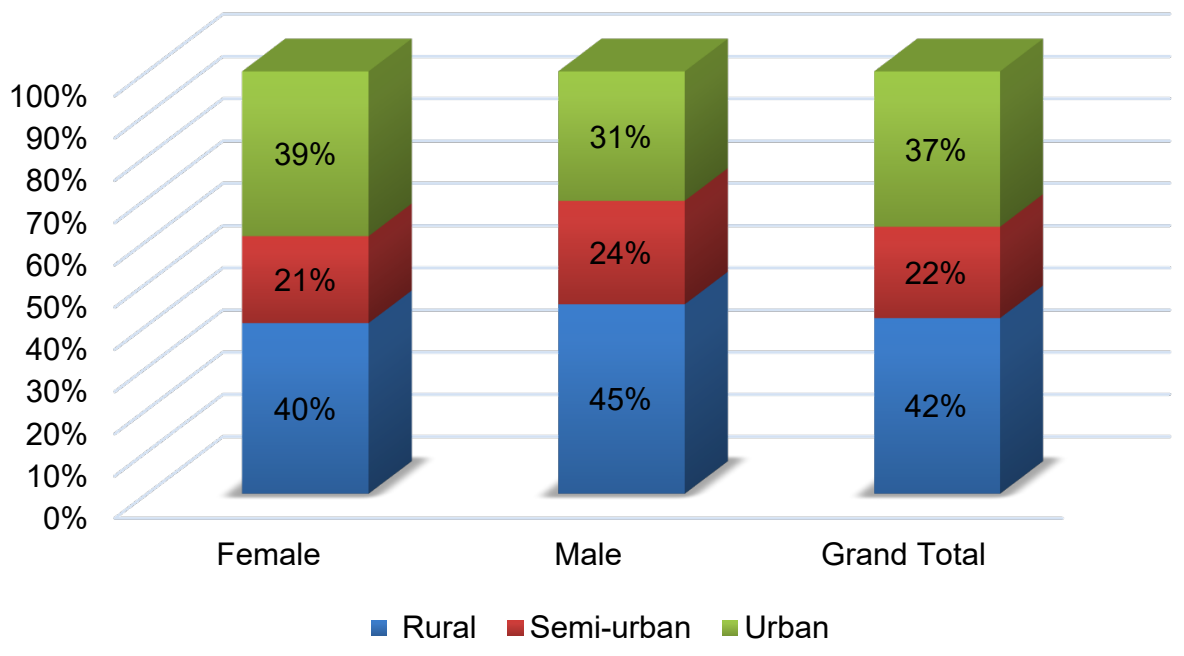

Other important survey demographics were that $84 \%$ of all male and $79 \%$ of all female respondents were married (Figure 4), and of those, $78 \%$ versus $87 \%$, respectively, were in monogamous marriages (Figure 5). 
Figure 4: Respondents' marital status

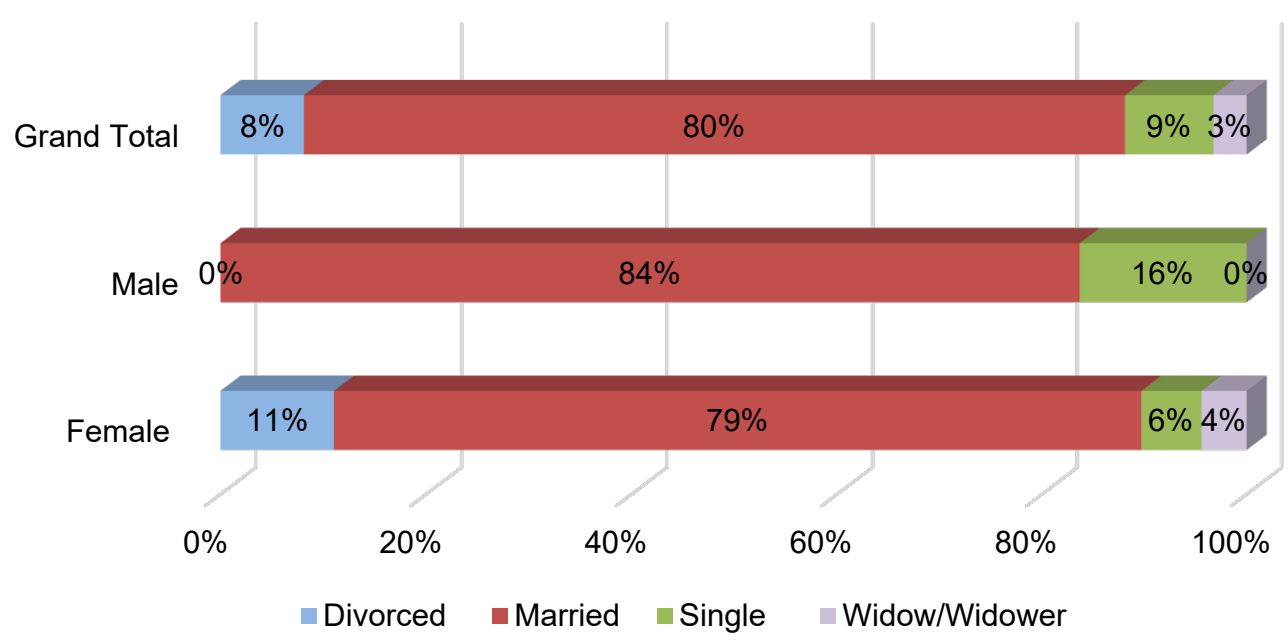

Figure 5: Marriage type

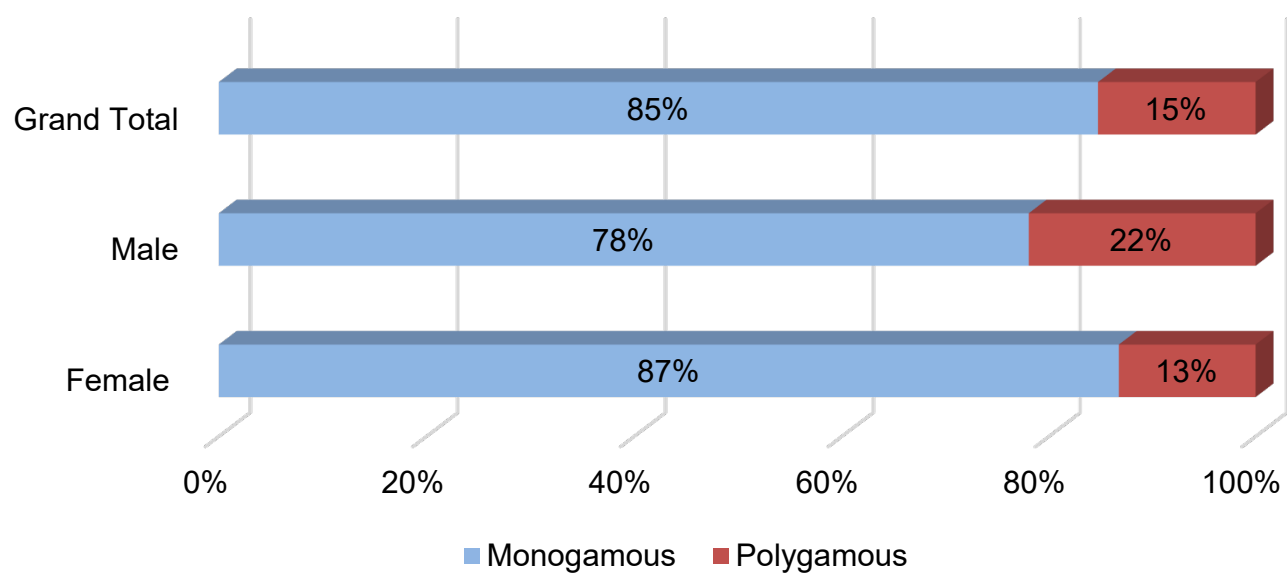

\subsection{CHALLENGES AND LIMITATIONS}

Although sufficient data was gathered across the three methodologies, the data collection team encountered small challenges due to COVID-19 related regulations, and several other issues. First, the duration of data collection, specifically KIls with the INGO and government representatives, was short due to the challenging situations at the time in the region (protests, COVID restrictions, and so on). Second, there were challenges in finding youth in one location due to their household responsibilities, and generally in finding community members for the FGDs due to farming and livestock responsibilities. Third, the enumerators mentioned that the time planned was not sufficient as there was a lot of information to capture and sometimes participants had competing demands, for example with the girls in the FGDs stating that they did not have more time to finalize the FGD due to household responsibilities. The enumerators would have also preferred longer training, although this was managed by the team through constant supervision, support, and feedback on each day of data collection. Nonetheless, these are important points to note for future gender analysis in the area. 
In terms of desk challenges and potential limitations to the study, a clear limitation was the lack of up-todate secondary data and general information on Puntland State specifically. First-hand information from local actors was used to address this challenge.

\section{GENDER ROLES AND RESPONSIBILITIES}

Gender roles are how society views and what it expects of people's behaviours and attitudes based on their assigned sex at birth. This can translate into the way a person acts, dresses, speaks, or generally interacts or conducts themselves in society. Traditional gender roles, focusing on gender-specific responsibilities, can accelerate or hinder a person's quality of life and access to services such as healthcare, education, employment opportunities, land ownership, and so on. In the FGDs, KIIs, and surveys conducted across three districts with both IDP and host communities in Puntland, participants were asked to share their thoughts on roles, responsibilities, and expectations of women, men, girls, and boys, using the framing of previous and current crisis circumstances. A compilation of the gender disaggregated findings is below.

\subsection{WOMEN}

It is evident through the FGD responses that women are considered a foundational pillar to many within Puntland, seen as the adhesive that keeps households functioning day to day. In numerous FGDs, women were referred to as 'the backbones' of their homes and communities. Across all discussion groups, women were acknowledged as the people responsible for household responsibilities, such as cleaning, childcare, and cooking. Furthermore, women are expected to manage internal roles in the family and provide guidance for children's behaviours and attitudes, as noted in the girls' FGD in Bariga Bosaaso. Girls in the Jowle FGD also highlighted women's role in solving conflicts between family members and guiding children's activities.

\section{Figure 6: Type of household head}

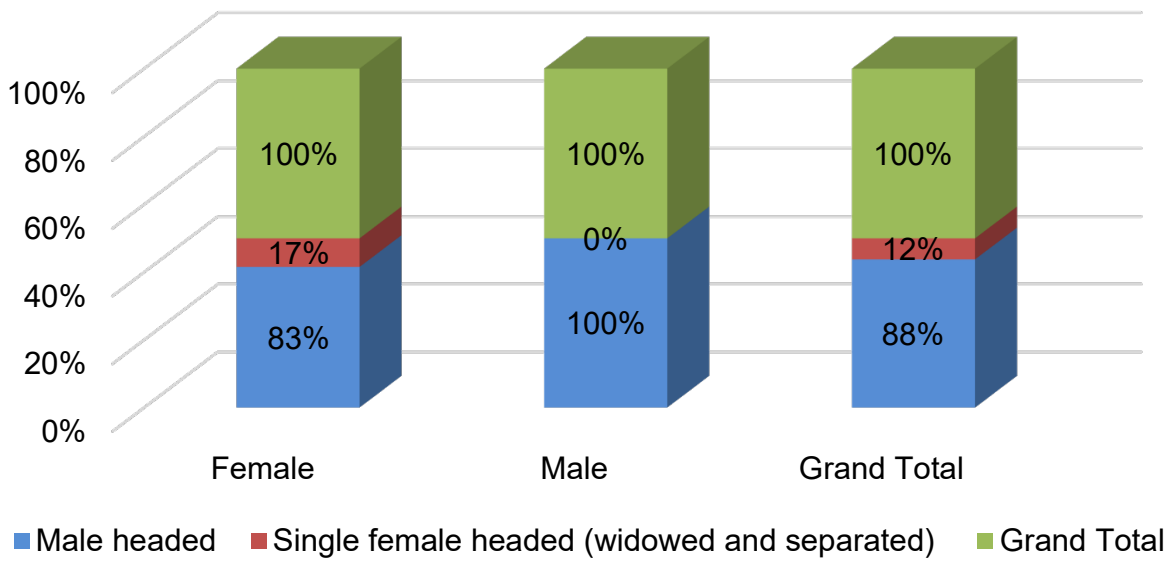


Nonetheless, the head of the household remains the man, as confirmed by the majority of FGDs, the survey data, and the KIls with community members. Figure 6 shows that $88 \%$ of respondents are part of a male-headed household, notably all male respondents, and with $17 \%$ of female respondents being from female-headed households. Before the current crises, women were often spending most time inside the house, noted by the IDP men's FDG in Bulo Migis. However, more are now involved with work outside the home, such as cleaning homes, washing clothes, and nannying for other families as a means to earn income. This group also noted that women's skills reside solely within the duty of care, and that they make money from that particular skill.

All four sites for the boys' FGDs (Bulo Migis, Bariga Bosaaso, Kalabayr, and Carta) saw women's role as that of household task management, which includes cooking, cleaning, and supervising children. The notable difference between the IDP communities and host communities is that in IDP settings, women now work and bring in most of the household income. Men in IDP settings also participate in household activities and help women while they are away from the household or busy with some of the activities. In the host communities, only in Geri Hel was it mentioned by men that they now collect water and firewood with women. In other aspects of life, there is no major change in gender roles in the host communities in the current crisis.

The survey results show that women are the ones responsible for all household chores, with most respondents stating that women are responsible for collecting firewood (Figure 7), overwhelmingly responsible for cooking (Figure 8), cleaning the house (Figure 9), and for taking care of children (Figure 10).

Figure 7: Firewood collection responsibility

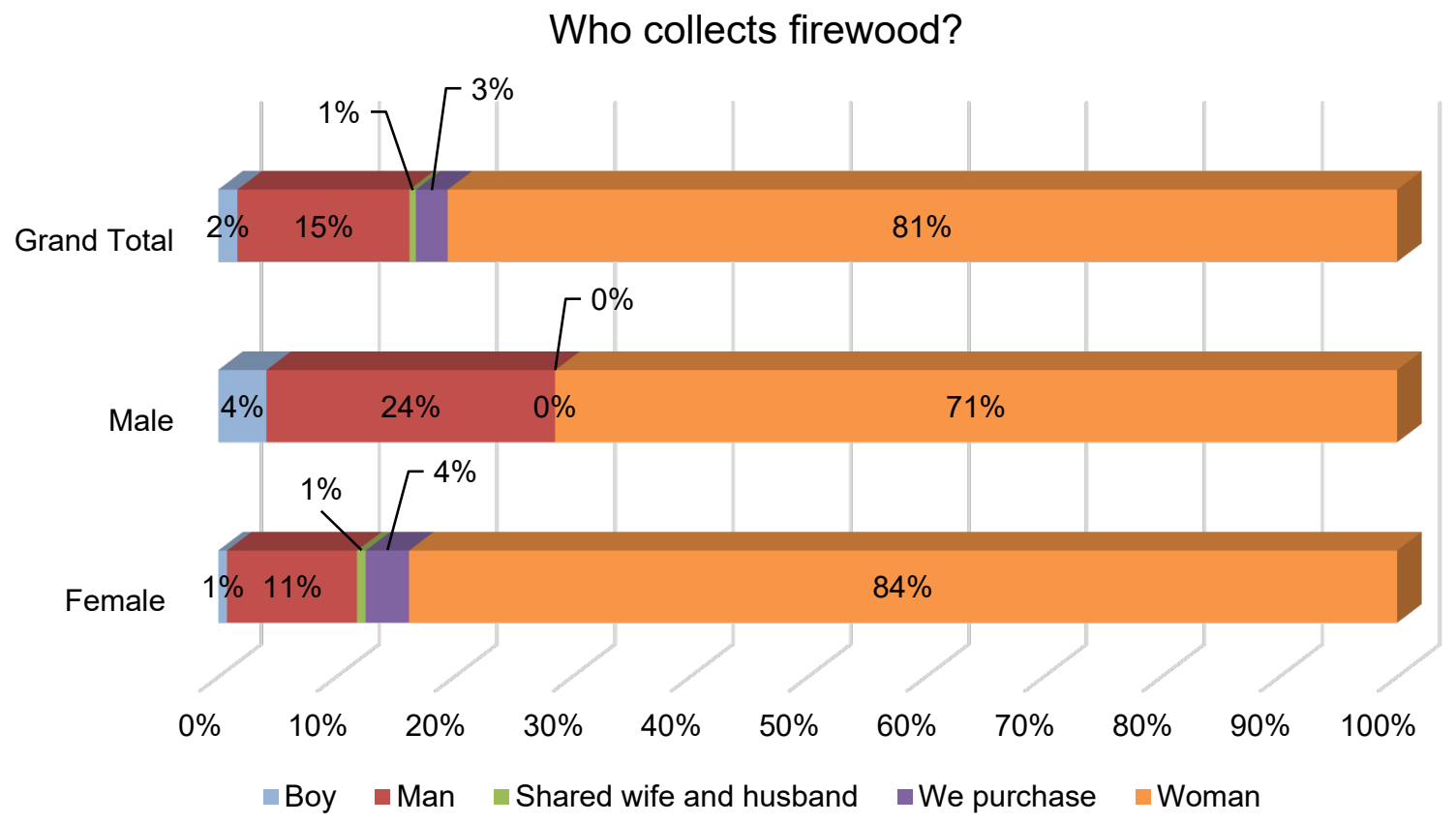


Figure 8: Cooking responsibility

Who is responsible for doing the cooking?

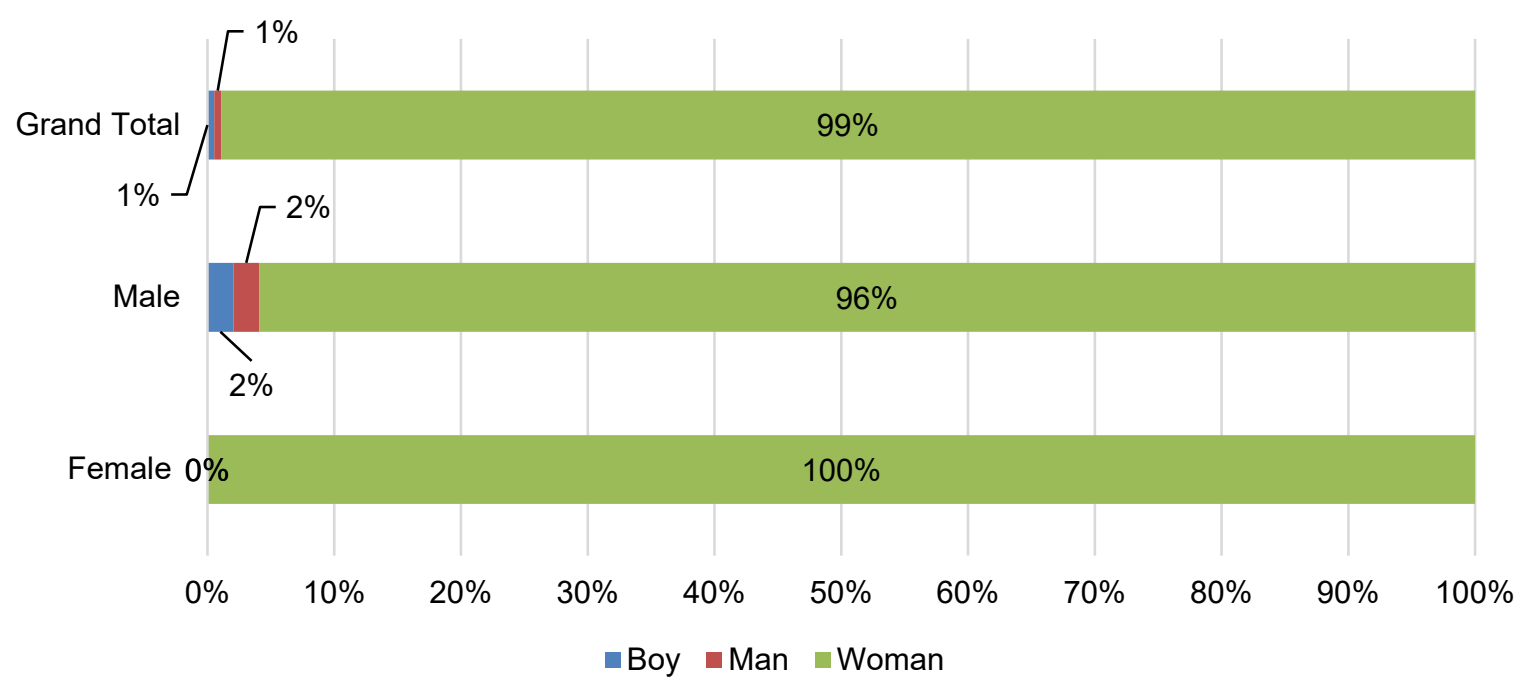

Figure 9: House cleaning responsibility

Who cleans the house?

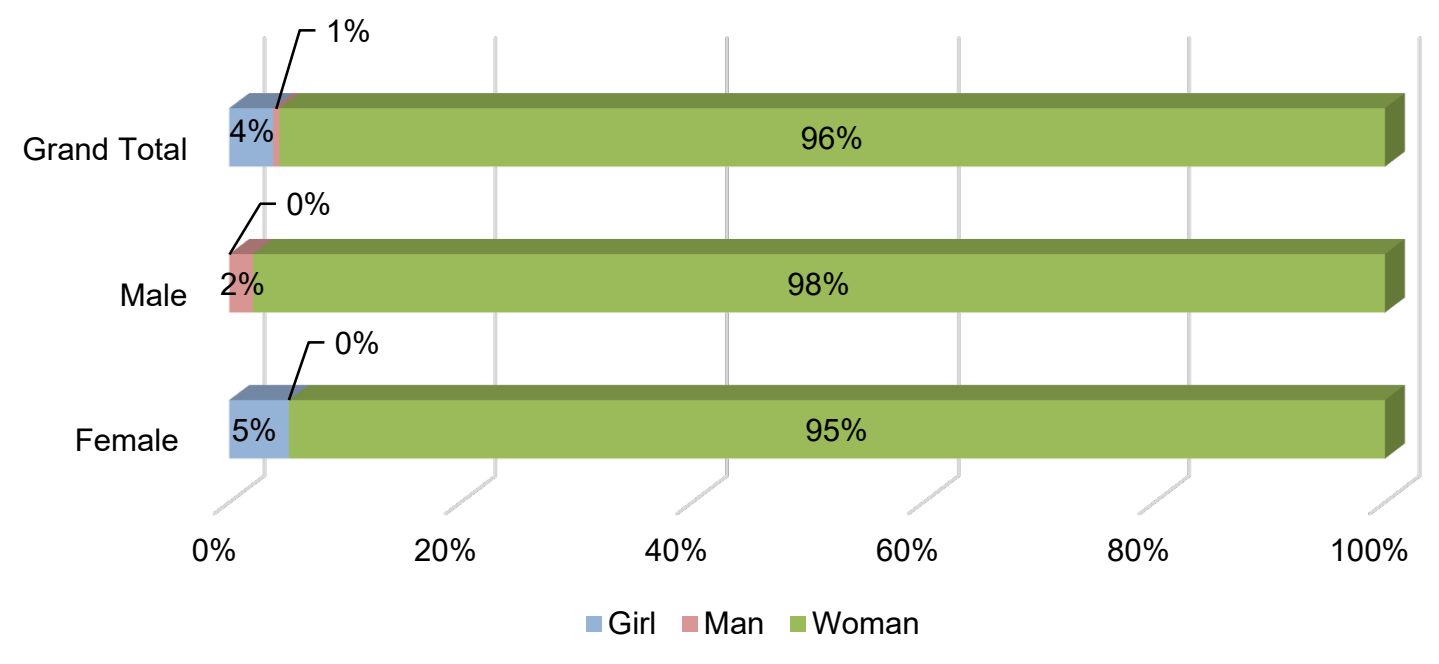


Figure 10: Childcare responsibility

Who looks after the children?

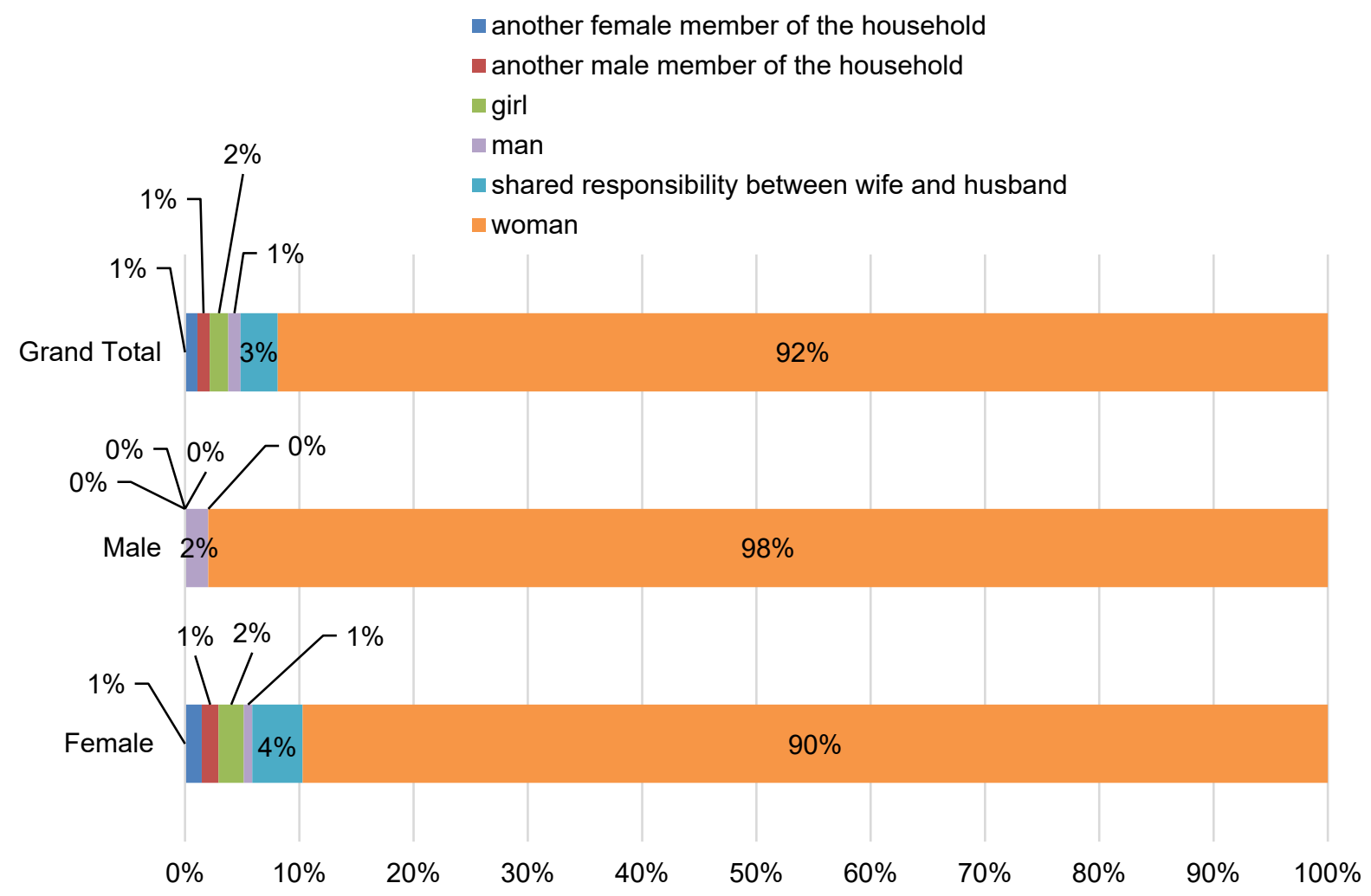

The FGDs and KIls confirmed that it is the men who have decision-making power. However, the survey highlighted some differences between women and men on these questions. For example, on the decision of their children's marriage, the men overwhelmingly responded that they decide ( $86 \%)$, with $45 \%$ of women saying it is the men's decision, $29 \%$ a joint decision, and $18 \%$ the women's decision (Figure 11). Similarly, on deciding on their children's school attendance, again men overwhelmingly answered it was them $(71 \%)$, but only $39 \%$ of women said it is the men and $26 \%$ said it is a joint decision (Figure 12 ). 
Figure 11: Decision making on children's marriage

Who makes the decisions on children's marriages?

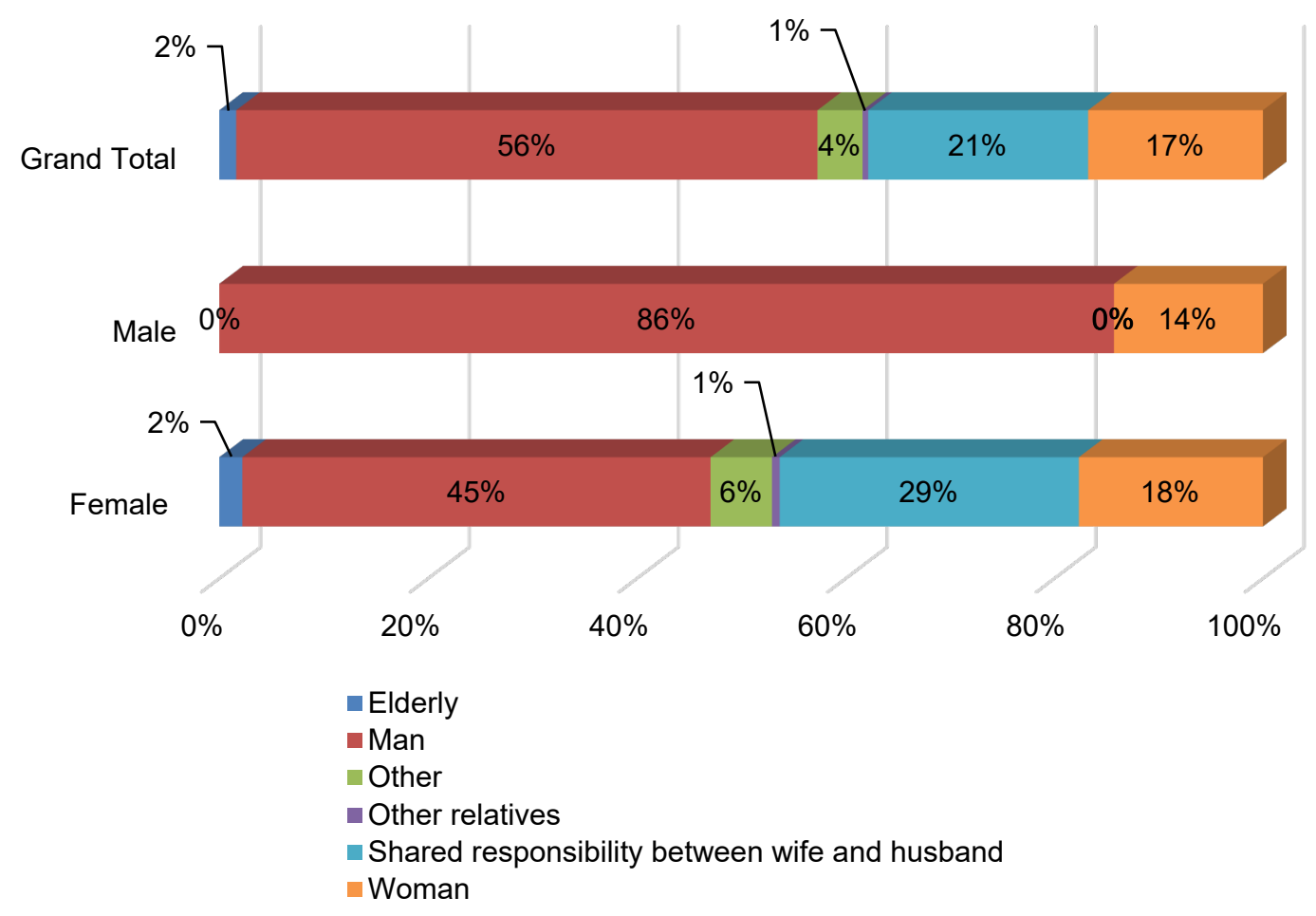

Figure 12: Decision making on school attendance

Who makes decisions on whether children attend school?

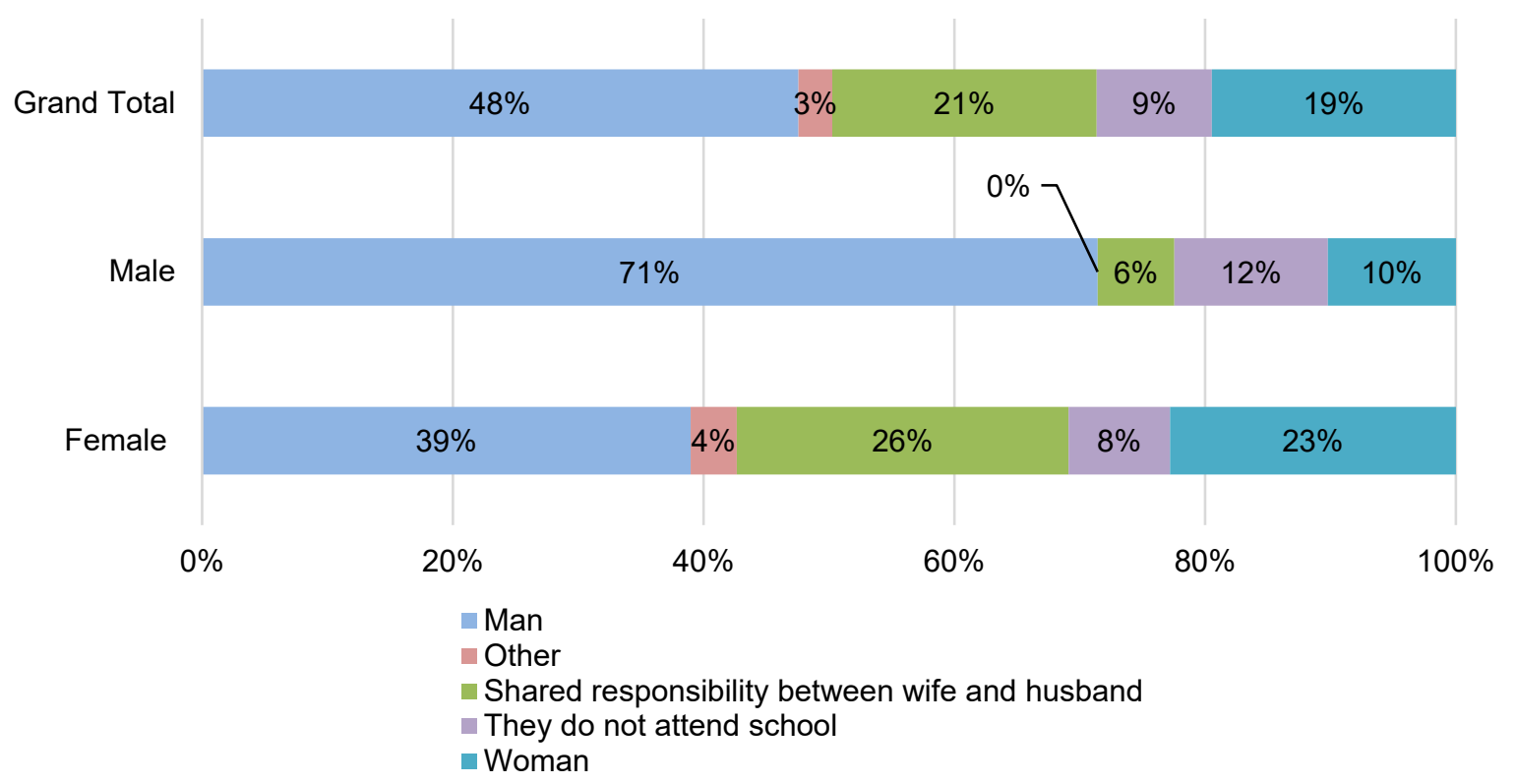

The reason for these discrepancies in the answers of women and men may be twofold: one related to the fact that the women were female heads of households or that what the women consider a joint decision, 
the man considers theirs alone. Some FGDs also pointed to women's household management skills. For example, in the boy's group in Kalabayr/Carta, women were seen as the primary holders of household concerns, financial management, leading on social activities, hospitality, and liaising with those who are interested in learning more about the community through formal women's committees. According to girls in Jowle, controlling the family's resources and spending its income also rests with the woman. Men and women make mutual decisions on who is going to school, children's marriages, and seeking healthcare. Women manage household resources and coordinate family members; fathers lead the family and ensure the availability of food, water, and sanitation. Likewise, both the Bulo Migis and Bariga Bosaaso boys' FGDs noted that mothers make the household decisions, allocate assets and resources for the family, and control the spending. Similarly, the women in Bulo Migis recognized that on some occasions, for example the case of a sick child, a mother may supersede the husband's choice to utilize traditional medicine and take the child to a healthcare facility if she is able to cover the cost of the clinic. However, in Kalabayr, the boys said men are responsible for seeking healthcare for a sick child.

According to the men's FGDs in Carta, Saacada, Geri Hel, and Cuun host communities, women collaborate jointly on household expenses; however, men were ultimately responsible for oversight. In Kalabayr, the host men acknowledged women's active role in family spending, and in Kubo, the host men noted that women participate in financial decision making around issues regarding school, marriage, and healthcare in consultation with their husbands. Host community men in Budunbuto stated that some women run small businesses and manage their livelihood finances, and that household decisions are usually taken using a mixed approach.

In the FGDs, women mentioned that their contribution resides mostly at home, where they have more leverage and management around decision making regarding daily expenses, food, childrearing, and healthcare. Though most contributors acknowledged this opportunity, ultimately, as observed by girls in Bariga Bosaaso, men hold the power and decision making at most household levels.

This was further confirmed by KIls, who overall reported that women do household responsibilities, while men are the head of the family, with girls supporting their mothers and boys supporting their father's work. One male key informant, a clan leader, said that men are 'responsible for the overall family well-being, livestock herding and doing hand work', and another male key informant added that 'women can take lower-level decisions but some women are leaders of their families such as single mothers and women whose men died, they lead their families'. One, however, said that women's role has changed and that given the hard situation as a result of the crisis, women have had to start work, and another stated that there are families where decisions are taken jointly. Key informants further said that community decisions are largely in the hands of men, with only one female key informant, herself a community leader, saying that women also participate in community decision making. Across the locations and communities, unless the woman is a female head of household, it seems that women's roles are relegated to household chores, with men being the decision makers at both household and community levels. These findings were further confirmed in the FGDs with men.

\subsection{MEN}

Men's roles were seen as those of protector, provider, problem-solver, and the ultimate arbiter of all things related to the household and family. The girl's discussion group in Bariga Bosaaso communicated that men are seen as motivators of their children's spirituality within the home and work to meet household basic needs, and are seen as problem-solvers both inside and outside the home. The girl's 
group in Jowle saw men as the supervisors of all household maintenance and the people who prevent risks.

Men were further seen as the religious compass for households and communities. For example, in Al Khayraat the girls said men taught the Quran, in addition to good household manners, and would advise the children to go to school. In Bariga Bosaaso, male IDPs outlined their role as religious teachers of the Quran.

In Bulo Migis, the boys' FGD noted that though men make the ultimate decisions, they do not intervene in issues around the home. Men also guide political issues and boys help both parents, whereas girls may only help their mothers.

From the men's discussion groups, it was easier to see the transition of how they saw their roles and contributions before and after the multiple crises. For example, the IDP men at Bulo Migis said that after the crises, economic and care work became more of a burden on both parents, and that men now help with household work when women are away for work or business. Similarly, the men in Bariga Bosaaso explained that before the crises, they were farmers, fishermen, livestock herders, drivers, and now for the most part they are finding employment solely as casual laborers.

KIls confirmed that decision making at the household levels is done by men, with mentions of consultations within the family. KIls across communities suggested that household resources, despite now being scarce, were still managed by men (this is as before the crises). However, there were several mentions across different locations of men and boys now supporting with household work. A female leader in Al Khayraat added that they help with fetching water, preparing firewood, and cleaning the house, although a male leader in Bulo Migis suggested that only some of the unpaid care work is performed, and only by a few men.

In the host communities, there is very little change in gender roles, with a female leader in Carta saying that there is no change at the household level, and only in Geri Hel was there one comment on men supporting women with household work. The few role-sharing examples should be used as positive role models across communities.

\subsection{GIRLS AND BOYS}

Both boys and girls are expected to contribute to the functioning of the household, but in different capacities and with varying demands on time allotted for those chores. In all groups, girls were viewed as the mother's helpers when it came to household tasks. Before, girls may have gone outside the home to do errands, but as explained by the male IDP FGD participants in Bulo Migis, they had to stop sending girls to the market after there had been protection issues. Girls were becoming victims of violence during their assigned work like doing laundry and cleaning for other houses, or helping mothers with their businesses in the city, so the tasks assigned to girls were now just those in their own homes. Further GBV and protection issues are highlighted in subsequent sections of this report.

Though it was not commonly mentioned that boys perform household duties, the girls' FGD noted that boys help with collecting firewood, carrying water, and buying items in the market, and furthermore, though some boys go to school, many work as shoe shiners, transitioning to car washers and bus drivers as they get older. In Al Khayraat, the group acknowledged that boys take care of the younger children and try to contribute to the family's income when they can. 
Boys in Bulo Migis and Bariga Bosaaso said that they help with housework. They knew their mothers were tired due to care work and income-generating activities (Bulo Migis), and they help financially and specifically with errands that need energy or strength, such as water collection, because the women are 'very weak'; but they love and respect them and feel they need their assistance (Bariga Bosaaso). The Kalabayr and Carta boys' groups noted they do not help that much with housework.

Boys protect their siblings and assist parents in their daily activities, according to the Bulo Migis group. Girls assist their mothers with household tasks, and in Kalabayr and Carta, girls sometimes represent their mother when it comes to decision making on certain issues.

Involvement in decision making was noted more so for boys, where they provide support to solve financial and food issues, while girls assist their mothers in duties around the home. Boys have more flexibility to go out and meet up with peers than girls, who tend to stay inside and attend to chores. Before the crises, girls had limited decision making and problem-solving involvement at the household level. They were always hidden and never really allowed to integrate with men nor boys. However, since they started helping their mothers, the eldest girls have power to control household assets in the same fashion as their mothers, particularly since mothers depend on them when they are sick, giving birth, or have a large workload. During the current crises, there were some mothers who left the country through illegal migration and girls automatically took on the maternal role. Some were asked to help mothers in their domestic businesses, while others stayed in the house to look after other children and the household.

Boys and girls both talked about the time they have for rest. In Bulo Migis and Bariga Bosaaso, boys' rest and sleep hours varied depending on the demands of the day. Only one boy in the two sites was a student and studied about four hours a day. Regarding time allocated to work, this varied in Bariga Bosaaso depending on the completed tasks, and in Bulo Migis one respondent noted he worked five to six hours a day while the others did no paid work at all. For the girls, the amount of sleep ranged across the three sites from eight hours a night in Bariga Bosaaso and Budunbuto, to seven hours in Jowle. Housework varied from six to eight hours, to unlimited hours according to the Jowle girls' FGD. This shows that similarly to the household roles and responsibilities that men and women share, the burden of care work falls on girls specifically.

\subsection{COMMUNITY PARTICIPATION AND DECISION MAKING}

When considering participation and decision making before the crises and in the current context, interviewees noted small shifts. The IDP men in Bulo Migis acknowledged that previously the men were ultimately making community decisions alone (with no involvement from women) but recently, displacement has forced men to include women in community decision making. There are more mutual conversations and consultations because the crises have made life more challenging. Before, women were not in the same spaces in terms of community issues and decisions; now, after the crises and displacement, women solve conflict at the household level and are invited to community meetings when their presence matters as they are earning money (it was noted that sometimes they earn more than men). Men in Bulo Migis also shared that with the recent arrival of more NGOs, there is a focus on listening solely to the women in the community. However, these men also believed that women have little power to take decisions, but those women who work and contribute to the family have the power to make some decisions at the community level. 
Roles holding respect and power among the community such as community elders, religious leaders, and those with elite status still reside with men across the communities, with the majority in the KIls with community members stating that while women are consulted, the decision stays with men. Where women do not have a say in community decisions, care burdens and income-generation activities also keep them busy and prevent them from participating in communal aspects. Numerous discussion groups raised the issue of engagement of women in community outreach, such as awareness campaigns and peace promotion.

Boys and girls do not have a role in community decision-making processes, though they will provide support for decisions at the household level. Boys in Bulo Migis did not have a role in the community before the crises, but now they have a very limited presence and role, as some go and work to assist the family. Therefore, they are asked to participate in some meetings, because they earn money and can contribute to solving problems.

Women's participation and role in community decision making was also brought to light in the host community FGDs. For example, the host men's discussions in Cuun and Kubo referenced women elders' participation in community decision making and in Carta, women were noted as members of the community committee. The host men in Kalabayr, Saacada, and Budunbuto emphasized the mixed makeup of village committees, consisting of both men and women. All the host men's FGDs confirmed that women leaders are present within their communities, although, for example in Kalabayr, where they exist, women leaders are rare.

The host community FGDs alluded to a more mixed group approach to village committees and decision making. Carta has two types of committees, a higher and a lower one, to work with community leaders, consisting of a mixed group of men and women. These committees take important decisions about the community and how to solve problems, in addition to enhancing the development of the community. In Budunbuto, Kubo, Geri Hel, and Cuun, community groups consisted of traditional leaders, religious leaders, elders, older women, and highly educated community members. In Kalabayr, host women acknowledged that they now have more authority in community decision making and mutual discussions.

The survey results suggest that both community women and men are largely excluded from community decision making, with this being the role of the local leaders only, as Figure 13 shows. Both in the survey and the KIls it was noted that there had been very little change in this situation since the compounded crises. 
Figure 13: Decision makers in the community

Who makes decisions in your community since the crisis started?

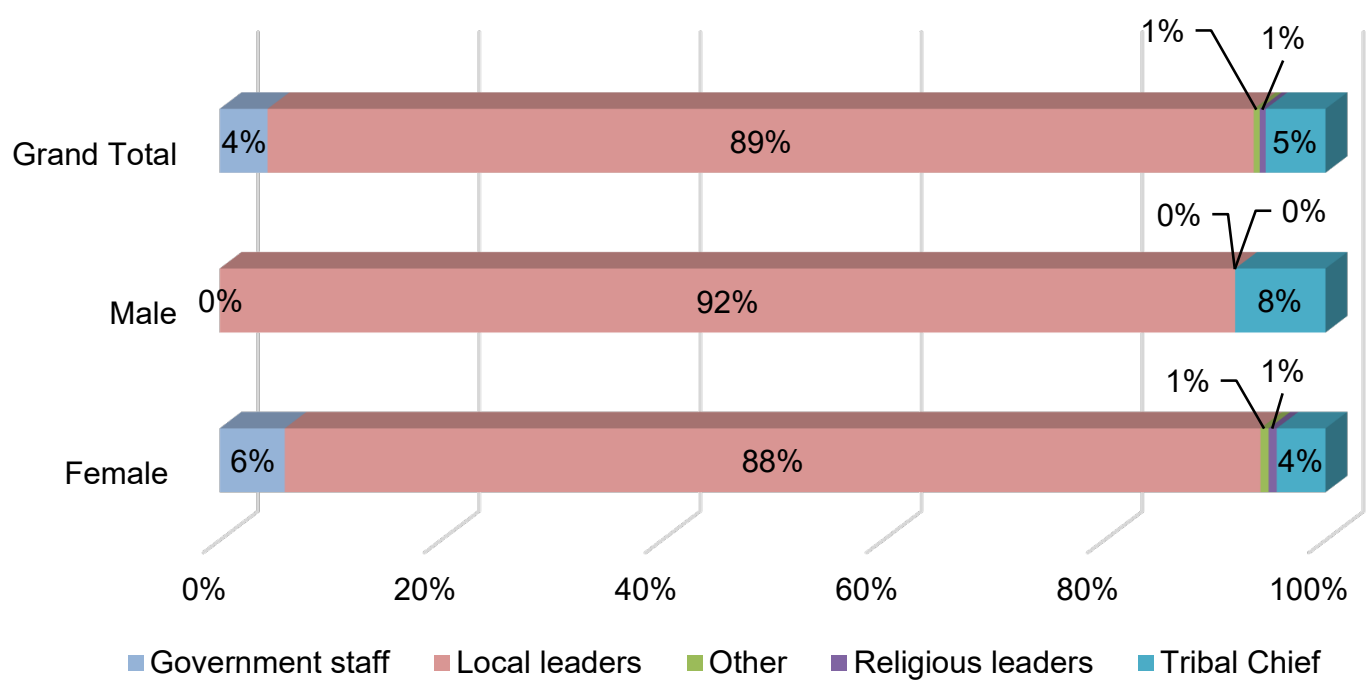

When asked why they do not participate in community decision making, $63 \%$ of the men and $43 \%$ of women said they are not interested; however, more women $(38 \%)$ compared with men $(31 \%)$ said they are not asked to participate (Figure 14).

Figure 14: Reasons for not participating in community decision making

Why do you not participate in community decision making?

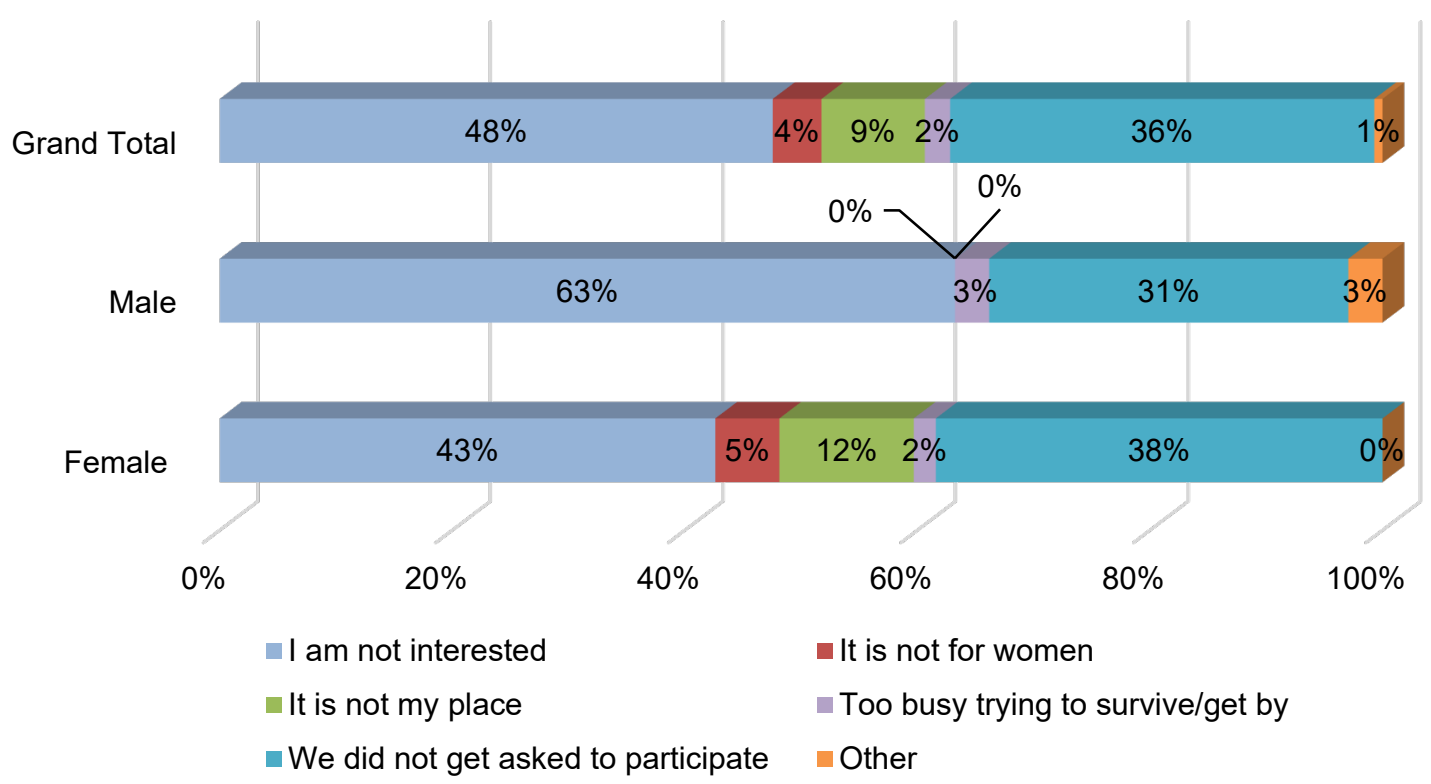

The community KIls mentioned that both men and women are involved in community consultations and are part of leadership structures, though two community chairpersons and one clan leader said that 'in our tradition women are not common in leadership structures'. Another community key informant further 
added that conflict resolution is 'ultimately the man's role' and that awareness campaigns and fundraising activities are performed by women.

\subsection{WOMEN'S LEADERSHIP}

There are some opportunities for, and examples of, women's leadership, with women leaders identified in a number of the FGD communities. The host women's FGDs noted that women leaders could be found in the sanitation, peace, reconciliation, and awareness raising spaces. Women leaders participate in all the community activities in the village and know their role in relation to the community needs. Combinations of informal and formal women's committees were recognized throughout the communities. In Bariga Bosaaso, men pointed out that the women leaders and organizations promote peace and conflict resolution, organize fundraising endeavours, and that their role has totally changed from housewife to worker (although they said that the women's organization is currently inactive).

However, the IDP men in Bulo Migis saw women's organizations as having no power at all - that they meet and 'waste their time'. However, the community committees (composed of all men) have agendas, action points, and tight deadlines, and the men meet for a reason and get something out of their meetings. As previously mentioned, all the male respondents believed that women have little power to take household decisions, but women who work and contribute to the family have the power to make some decisions at the community level. Some women mentioned they can participate in the community committee meetings as some of the members are women (though this was only the case in two locations), while girls and even boys never participate in or are invited to such forums and discussions. In Jowle, the IDP men said there are no women's groups, and some said that they do not believe women can be leaders, despite stating they are the backbone of their households and communities.

The girls and boys in Bariga Bosaaso said the women leaders work on family issues and solving disputes, and in addition, in Al Khayraat, women leaders also have that role in environmental mindfulness, monitoring IDP health conditions, and community awareness messaging for issues such as COVID-19. In Jowle, girls confirmed that the vice chairperson of the community was a woman, and women undertake community organization, mobilization, and awareness. They also make decisions at the household level. The boys in Carta noted that there are some women leaders, but they were unsure of what they do.

Women IDPs in Bulo Migis confirmed there are informal women's organizations in the community that contribute toward the safety and social needs of families and neighbours, and also play a critical role in solving internal household matters. Women hold essential roles in the community (promoting peace, solving family problems, organizing campaigns, children's schooling, and managing households) and organize women to come together to discuss their own problems and explore resolutions. In Bariga Bosaaso, women and men share leadership duties around decision making and problem solving. An FGD with women mentioned they have a self-help group which works as a savings group to help each other. Women are changing traditional gender roles by working and supporting their families during the current crises. According to the IDP women's FGD in Jowle, women's decision-making power mostly lies within the household sphere, and there are women leaders in Al Khayraat that work in informal settings but also contribute to community discussions.

In the host village of Kalabayr, men said that women can openly participate and that women leaders exist but are rare. The host men in Saacada, Geri Hel, Cuun, Budunbuto, Kubo, and Carta also confirmed the presence of women leaders. Interestingly the host women of Cuun and Budunbuto stated there are no predominant women leaders, but that women participate in community discussions. The women in 
Kalabayr, Carta, Kubo, Saacada, and Geri Hel explained the women leaders' roles in the communities as promoting peace, awareness raising, consultations, and fundraising activities for causes such as a mutual aid fund to help others in the community.

From the survey, few men (27\%) and even fewer women (14\%) answered that they were part of groups or associations (Figure 15).

Figure 15: Membership of group or association

Are you a member of any type of association or group that regularly holds meetings?

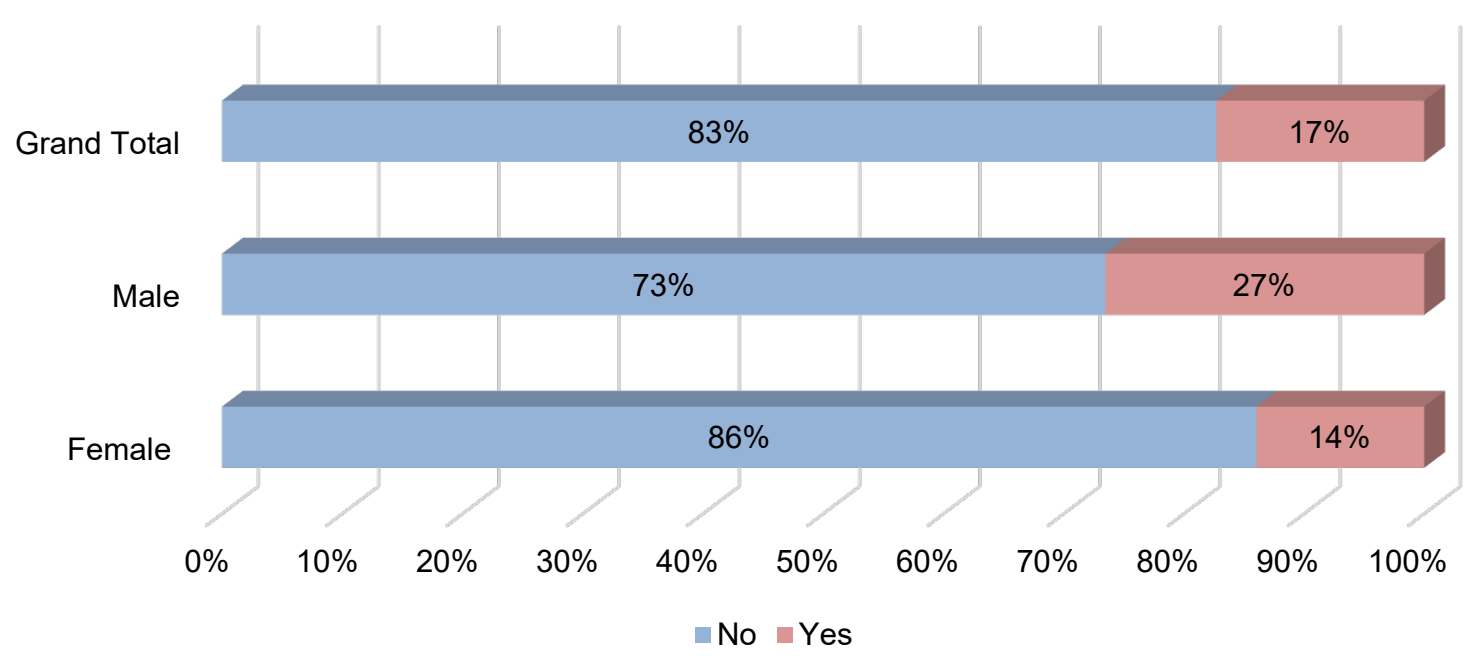

Of those who are members of groups, the majority of women (63\%) are part of women's groups (Figure $16)$, a finding supported by the FGDs.

Figure 16: Type of association

If yes, what type of association?

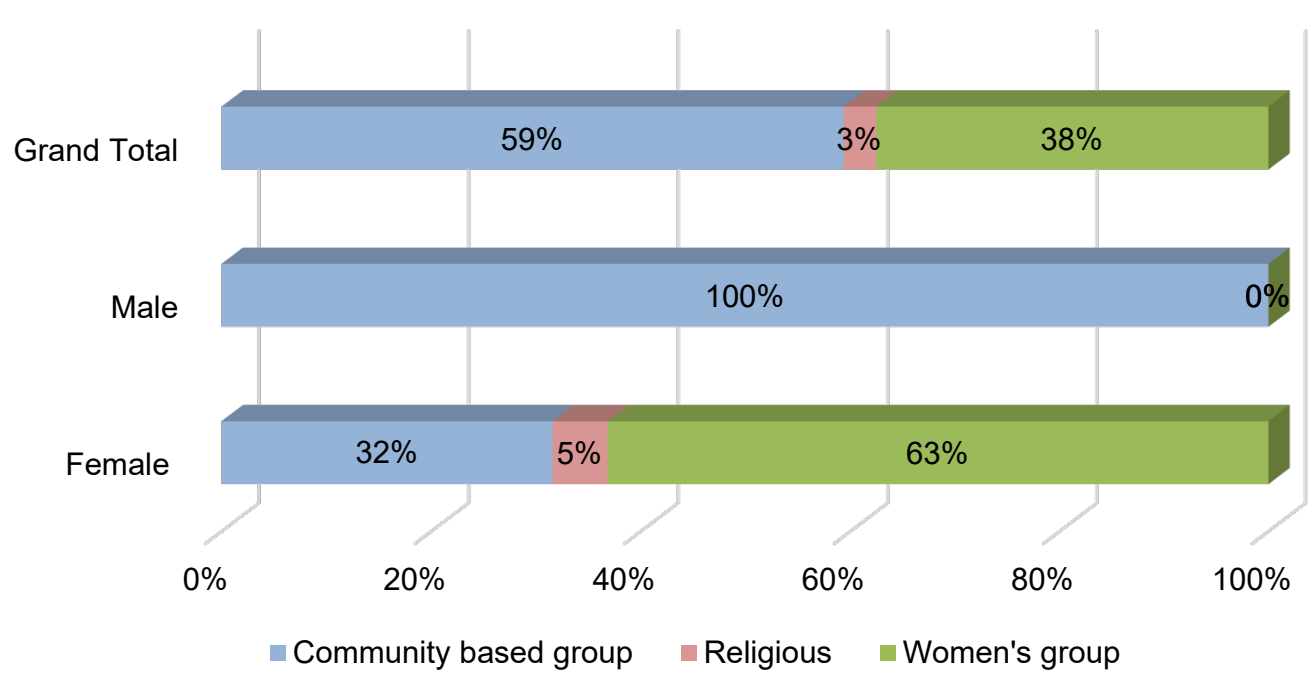


Across all the community locations, both male and female key informants mentioned that there are women's groups or women leaders in their locations. However, one leader each from Jowle, Kubo, Bulo Migis, and Al Khayraat added that the women's organization is not working effectively or is inactive because of a 'lack of knowledge, encouragement and support'. In Budunbuto, women are involved in awareness raising, and in Kalabayr, the women's group 'properly performs' and their recommendations are respected. When asked about the challenges to women's leadership, the community KIls mentioned several, key among them 'the community perception that women cannot lead'. One went further to say, 'women are not allowed to talk to men, let alone to lead'. A religious leader said that 'women are not allowed to lead', quoting the Quran. A woman leader argued that 'there is no investment in girls to do such work' and two male leaders said that they are too busy with household work, with two other key informants (one male, one female) adding that livelihoods and food insecurity are on women's minds first, and another adding that 'women do not have time to deal with big issues'. Several key informants mentioned a lack of education as a key challenge. Notably, one female leader mentioned that she is excluded by the leadership structure. The community key informant data show that there are several barriers to achieving women's economic empowerment - such as men's traditional beliefs that every productive job is for men and that, similar to leadership roles, women are too busy with housework to be able to have productive roles in the community.

\section{ACCESS TO WASH}

Safe and accessible mechanisms to meet water and hygiene needs are at a bare minimum in the communities. The FGDs, KIls, and survey all confirmed that potable water, secure latrines, proper lighting, accessible boreholes, and sanitary items are desired commodities for all. The survey found that $59 \%$ of men and $68 \%$ of women said they do not have access to clean water (Figure 17). In Bariga Bosaaso, water from the borehole is expensive, costing 3,000 shillings ( $\$ 1$ was equivalent to 55,000 65,000 shillings at the time of study) for 20 litres, the borehole is remote, and sanitation facilities are not available. FGDs with community members mentioned that community leaders first store sanitation tools and hygiene kits they receive in a storage area, and then divide them between households based on their vulnerabilities. There is not enough water for the community to use, and borehole and water reservoirs are needed. An additional common issue mentioned across all communities was the lack of water cleanliness, with children and women suffering from severe diarrhoea. 
Figure 17: Access to clean water

Do you have access to clean water?

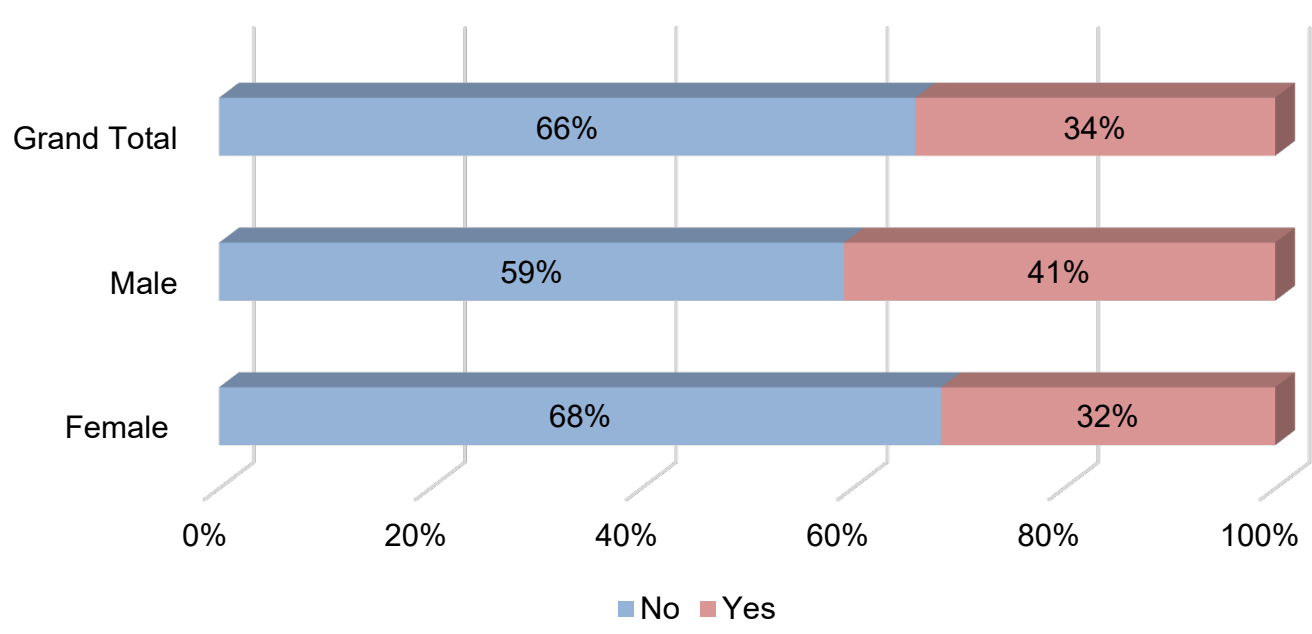

Seventy-six percent of men and $67 \%$ of women said they do not have access to hygiene items such as soap, sanitizer, or chlorine (Figure 18). The lack of sanitation tools and hygiene kits was also raised by women's FGDs in Bulo Migis. Men in the FGD said they received chlorine and handwashing soap as aid and use plastic bags to keep water in. However, this was a rare example of obtaining aid supplies mentioned by the communities.

\section{Figure 18: Access to hygiene items}

Do you have access to soap/sanitizer/chlorine?

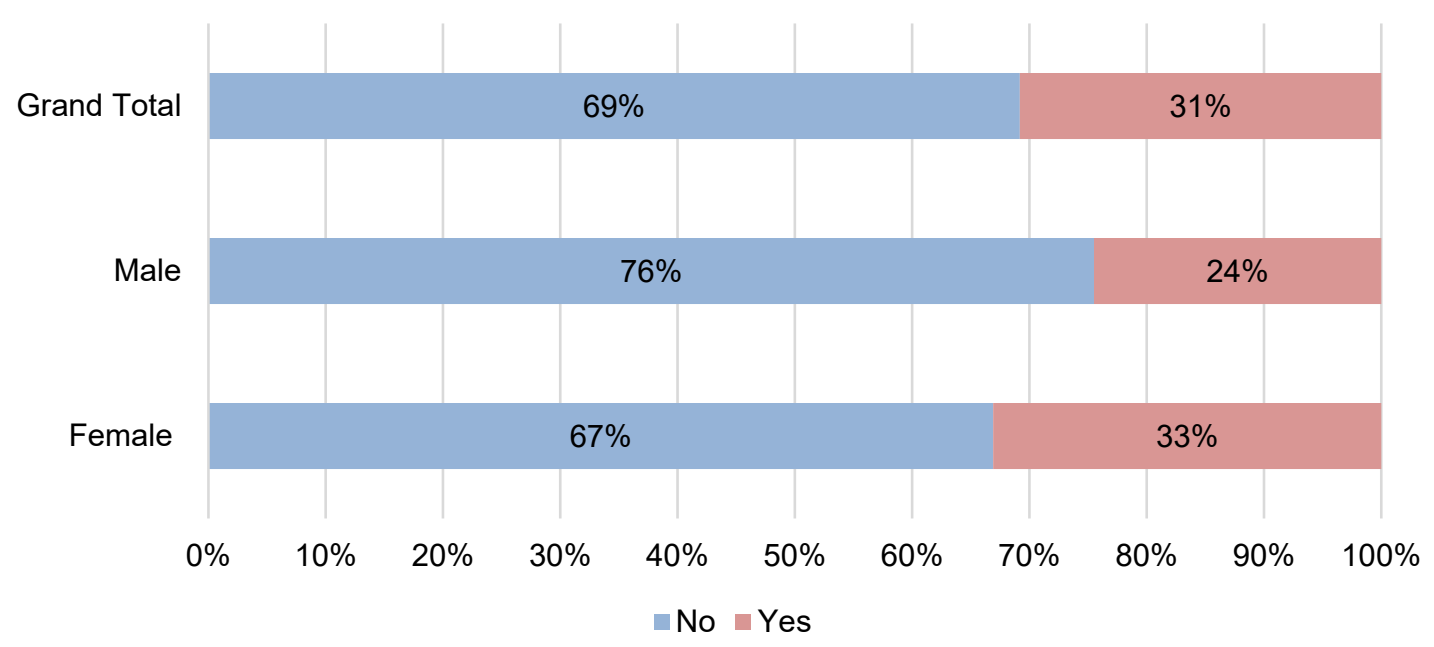

Furthermore, across all the FGDs, KIls, and the survey, women were seen as the ones who undertake the tasks and decision-making roles related to WASH responsibilities, in line with their care burden at home. When it comes to who collects water, Figure 19 shows that respondents answered it was the woman, according to $74 \%$ of the women and $69 \%$ of the men (with $24 \%$ of men adding that they also collect water, despite very few FGDs confirming this). 
Figure 19: Water collection responsibility

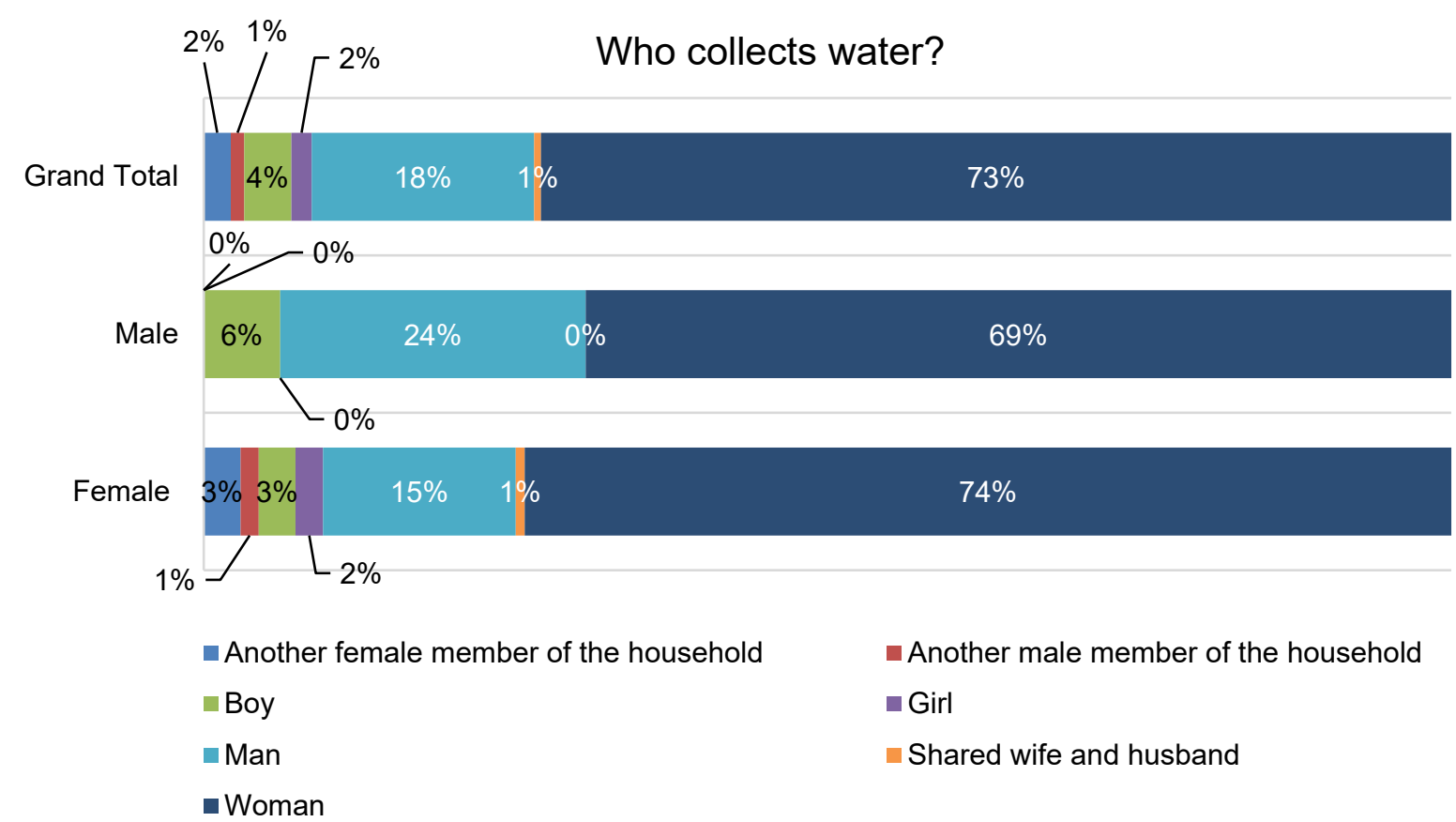

In terms of how long the water collection takes, $63 \%$ of women versus $94 \%$ of men said it takes less than 30 minutes (Figure 20). For $18 \%$ of the women it takes more than an hour every day to collect water once; coupled with the number of times they would have to collect water a day, the care burden for those women is particularly worrying. The FGD with women in Bulo Migis addressed some of the main WASH issues, these being the distance to the borehole and the physical strain of carrying water long distances and in large quantities. Furthermore, that there is not enough water for the community to use, no place to dispose of rubbish, and a need for water storage options instead of daily trips to draw water. There are no sanitation facilities available, and due to the lack of facilities and hygiene, there are sometimes disease outbreaks during floods as the water becomes stagnant and contaminated.

Figure 20: Duration of water collection

How long does water collecting take?

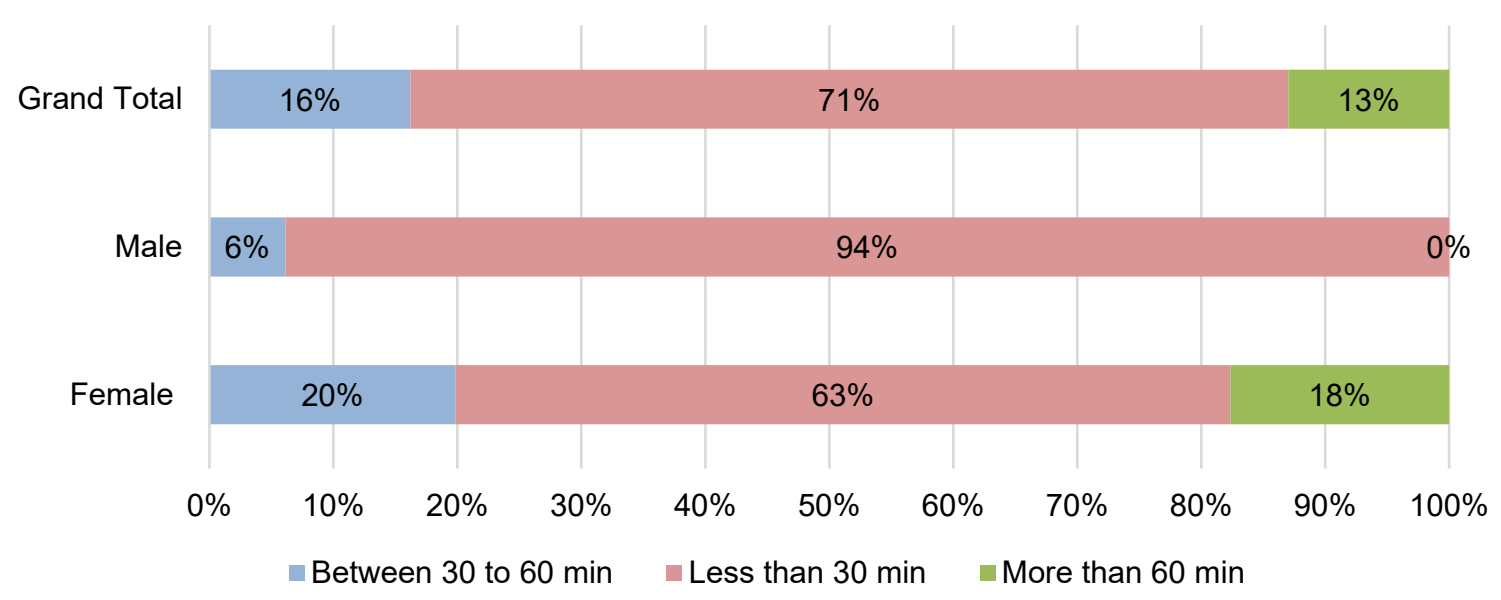


Women are also the ones who are responsible for disposal of waste, as overwhelmingly confirmed by the survey respondents (Figure 21). The FGDs with IDP residents in Jowle also mentioned that there are no latrines and they do not have sanitation facilities such as solid waste management bags, only plastic bags. They also confirmed that women are the key players for personal and family hygiene, waste management, and ensure the availability of tools required for sanitation. Men carry and supply the sanitation facilities and preserve water points. The main challenges in Jowle are that the water points are remote, there is no wheelbarrow to transport water, and the water availability is inconsistent. There are few toilets and those that are there are full and broken. The Garowe local government collects waste once a week. According to women in Al Khayraat, KAALO distributes filtered water for 75 households. There is no sanitation equipment available: however, they clean everything on Fridays and burn the rubbish in the valley. Women also manage the sanitation equipment in this community.

Figure 21: Waste disposal responsibility

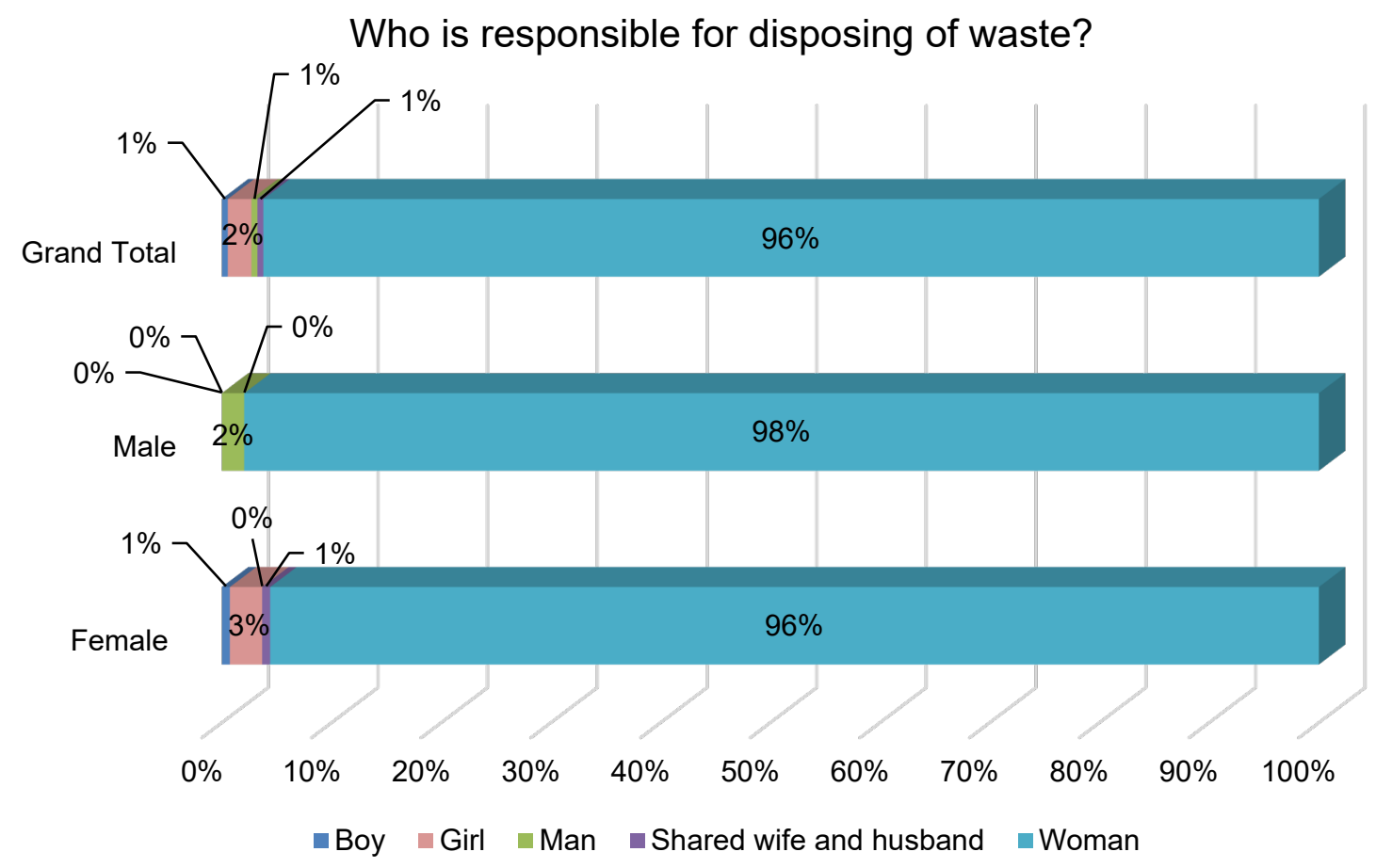

When asked whether the crises have changed responsibilities at the household level, $75 \%$ of the women said it had not (Figure 22). However, the $25 \%$ who said responsibilities had changed mentioned water collection as one of these responsibilities (Figure 23), showing that the compounded crises did affect access to WASH. 
Figure 22: Change in responsibilities since the crises

Have responsibilities changed since the crises?

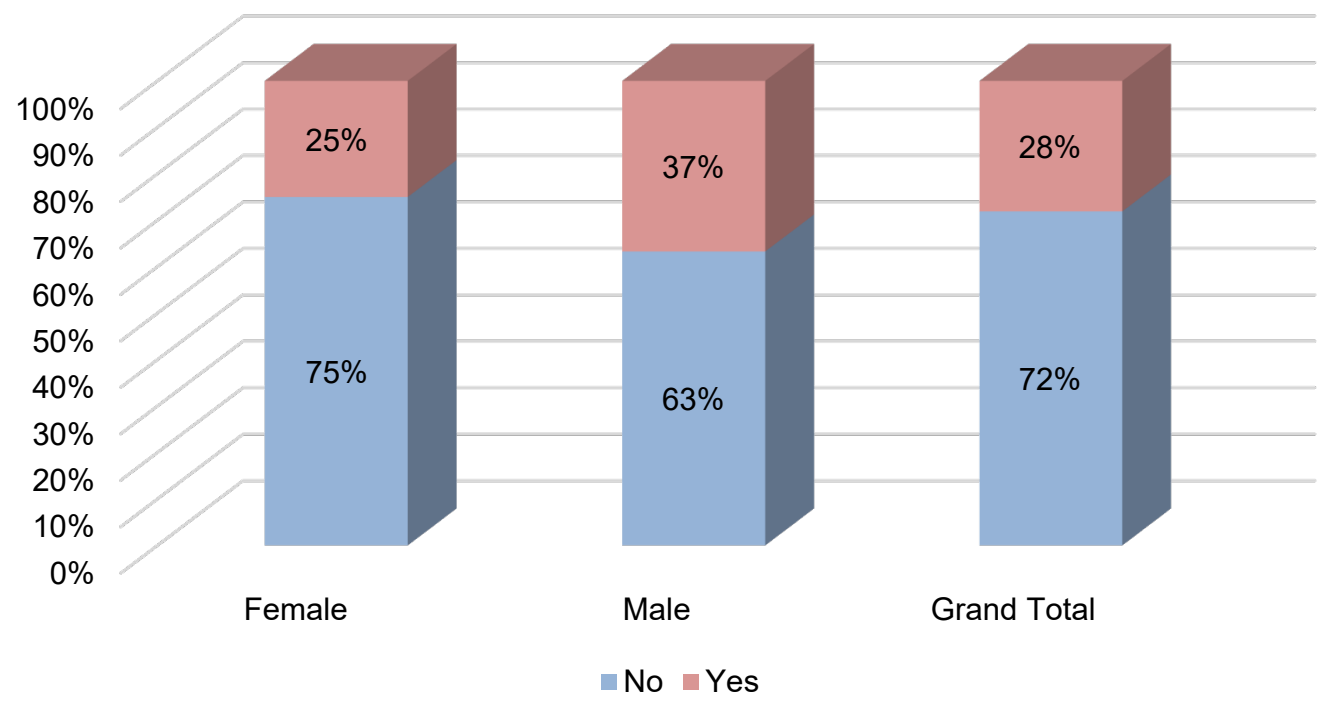

Figure 23: Details of which aspects have changed

If yes, what aspects have changed?

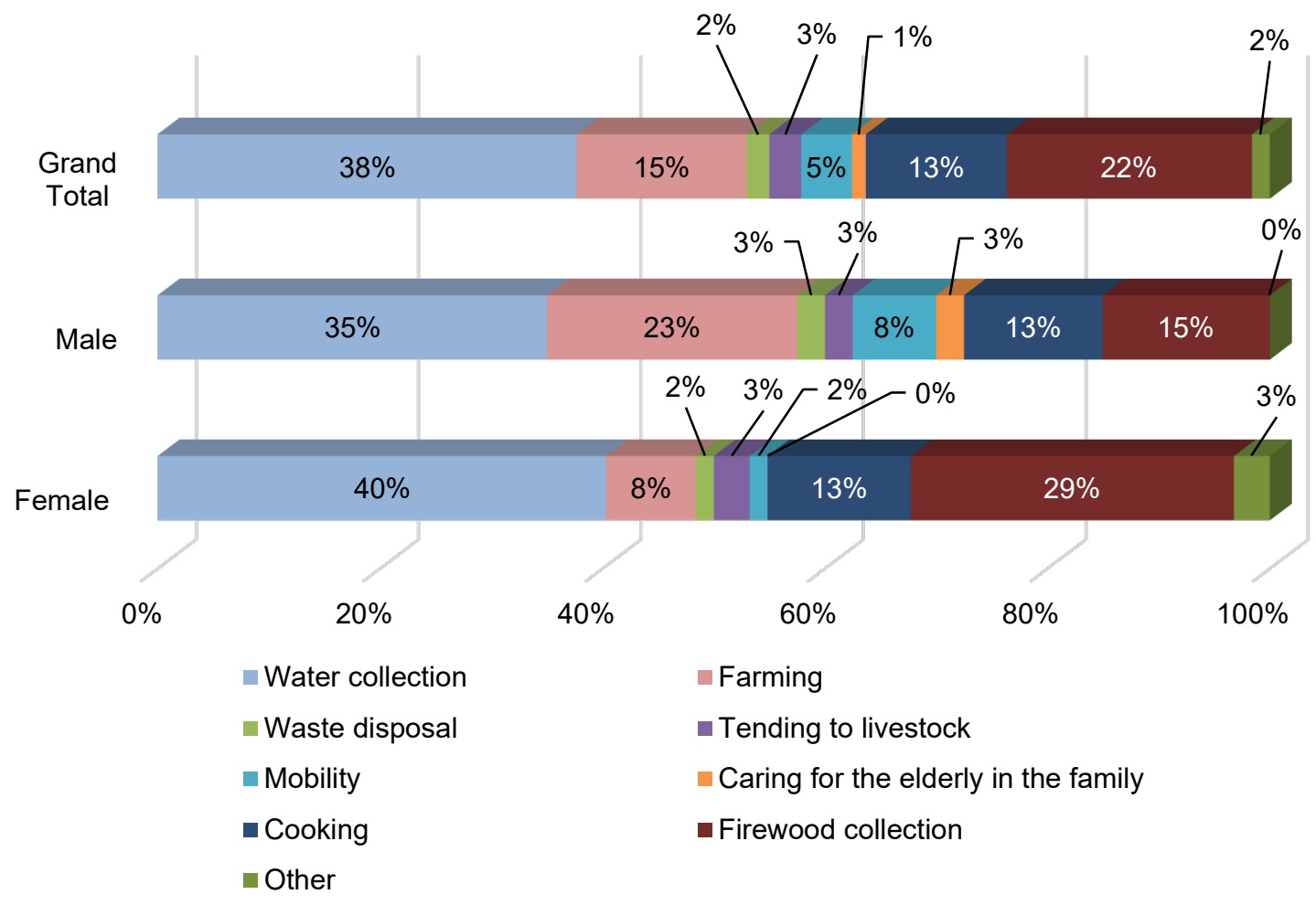

Safety is an important issue in relation to WASH. Across FGDs, the survey and KIls, the community, and in particular women and girls, mentioned certain activities that can put them in greater jeopardy. Fear for 
their safety while fetching water, using toilets, and bathing were raised in FGDs and confirmed by the survey results. Figure 24 shows that $57 \%$ of women do not find the existing water points safe. One reason for this is the lack of consultation on the location of these facilities.

Figure 24: Water point safety

Is the water point safe?

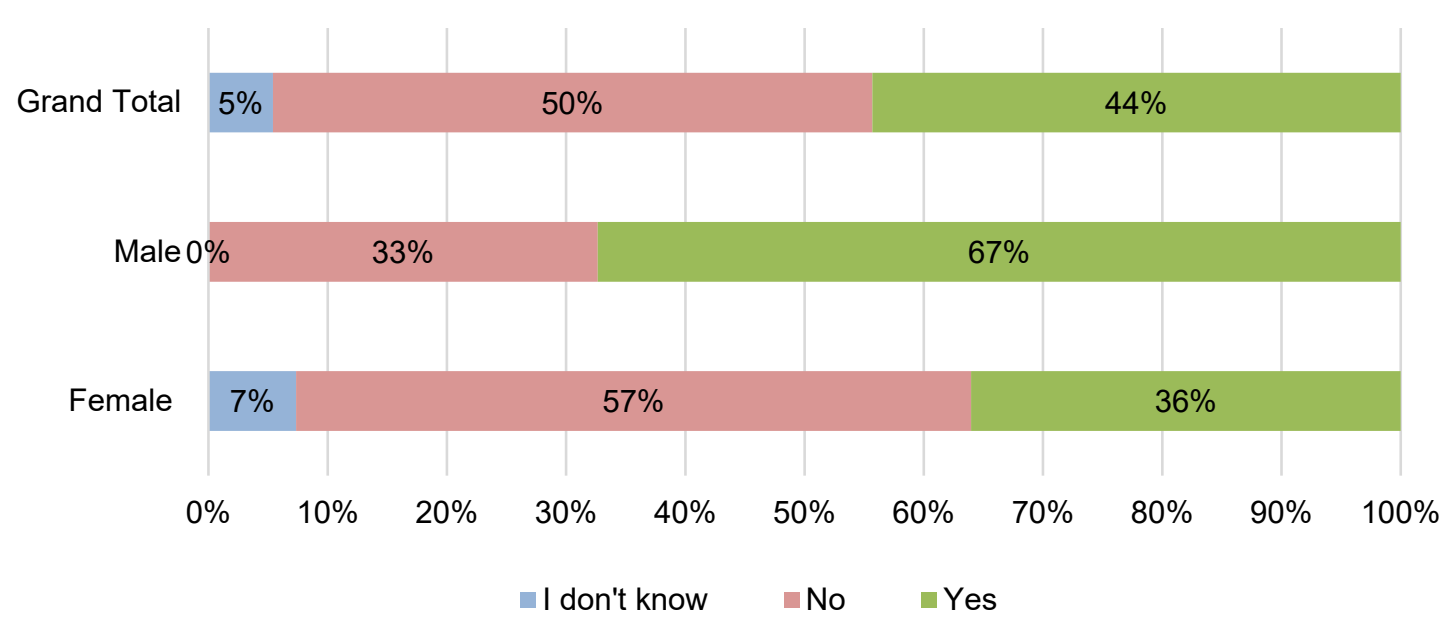

In terms of mechanisms to cope with the lack of safety at water points, women prefer to go early in the morning or in a group (Figure 25).

Figure 25: Coping mechanisms if water collection point is unsafe

If not safe, what are you doing to cope?

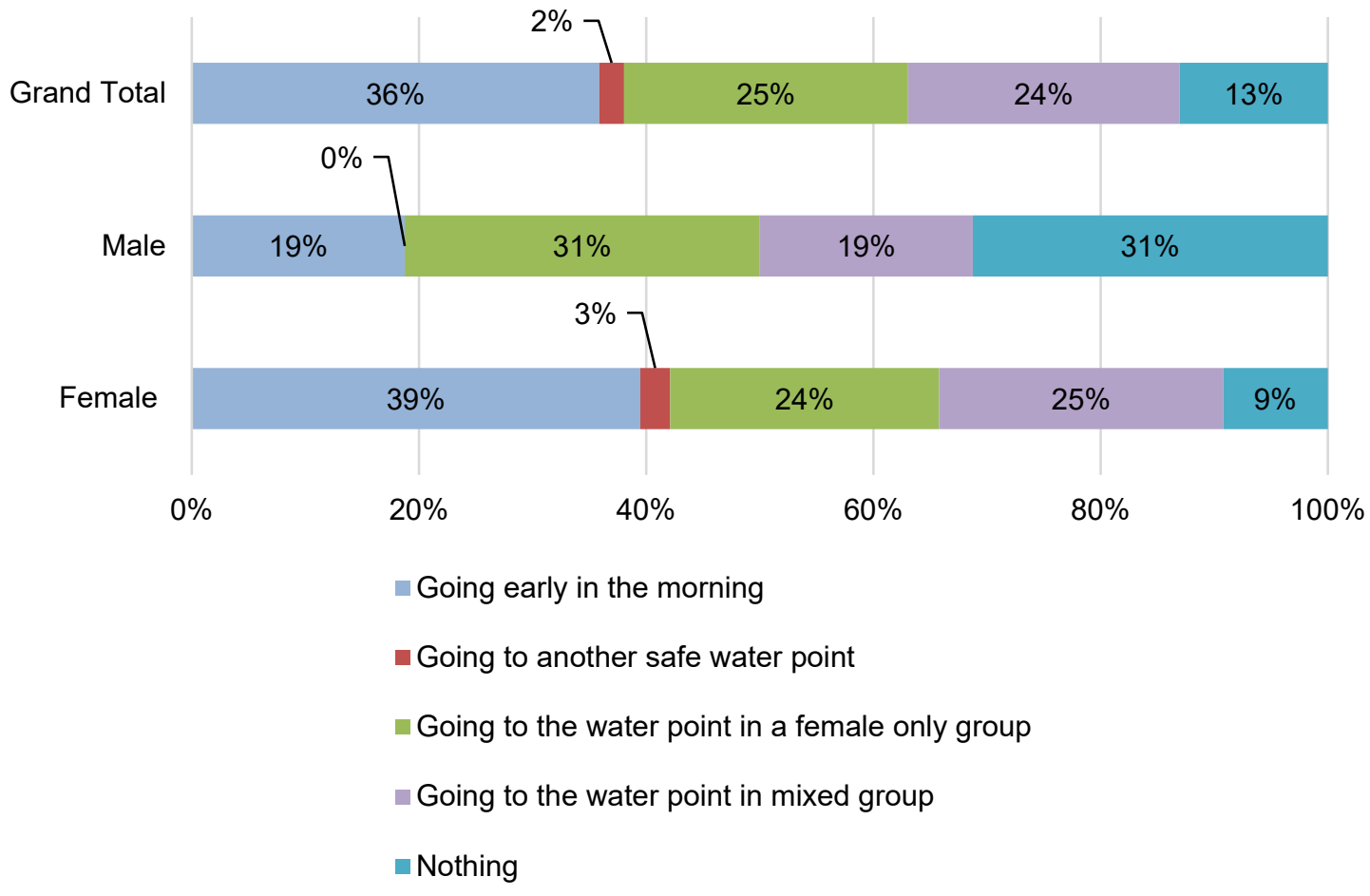


The IDP FGD participants further noted that in their settlements, toilets are far from many of the camp inhabitants and there are no lights. It is not safe to use the toilets at night, especially without a torch, which survey respondents confirmed. Women and girls need toilets in their homesteads, as well as lighting and water storage. In Bariga Bosaaso, the men conveyed that because the distance to the toilets is too great, people were openly defecating. In some cases, boys will hide and throw rubbish and stones at girls while they are walking to find somewhere for open defecation. The survey found that half of the women and $65 \%$ of men do not have access to a safe latrine, either because there is no latrine at all, or it is not safe at night (Figure 27). The Jowle IDP community is noteworthy, as the men in the FGD stated there are no toilets at all, as the landowner from whom the settlement land is rented refused them consent to dig toilets and wells.

\section{Figure 26: Access to safe latrines}

\section{Do you have access to safe latrines?}

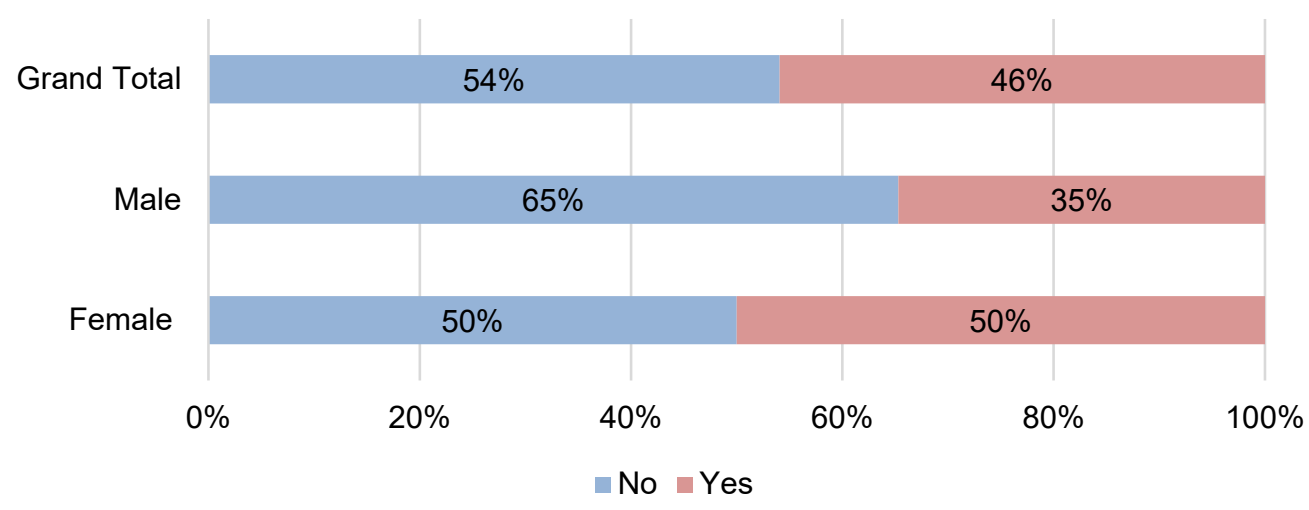

Figure 27: Reasons for a lack of safe latrines

If no access to latrines, what are the reasons?

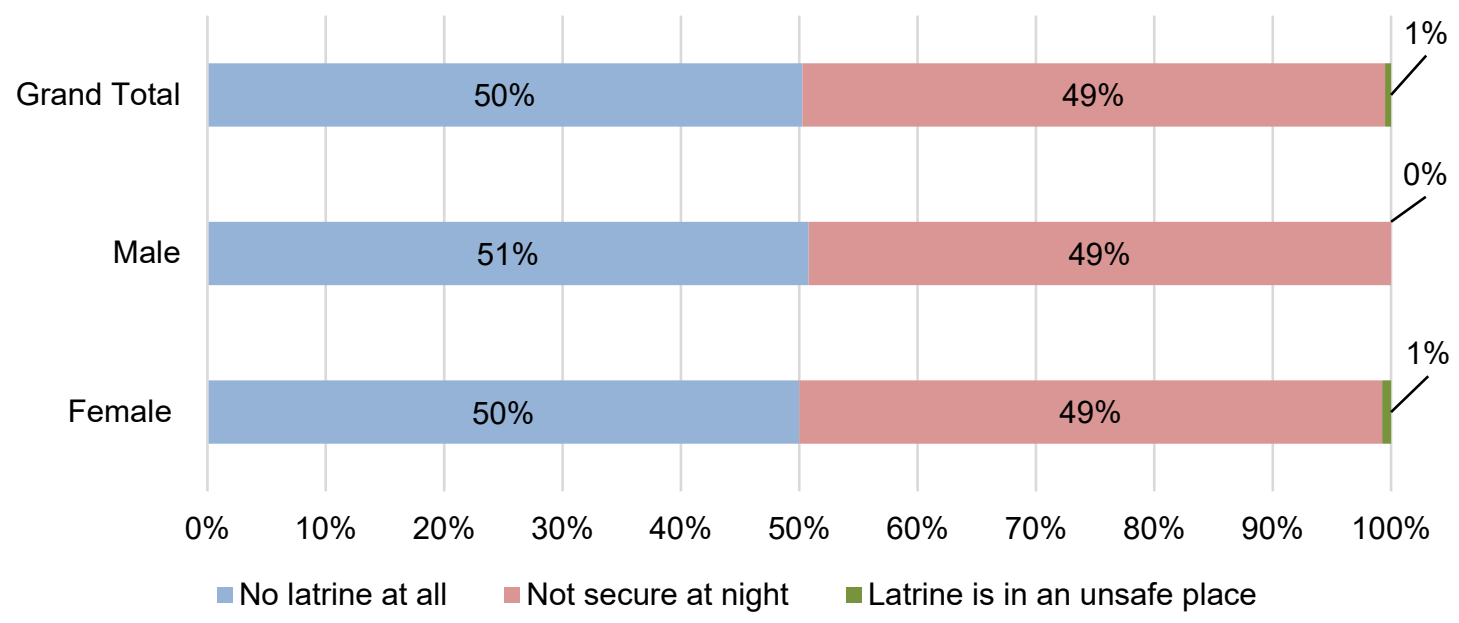

Over half of the survey respondents ( $54 \%$ of the women and $65 \%$ of the men) said that they do not have access to a safe area for bathing (Figure 28), primarily due to lack of a bathing space (Figure 29). This was confirmed by the community KIls and one INGO key informant. 
Figure 28: Access to safe bathing

Do you have access to safe bathing?

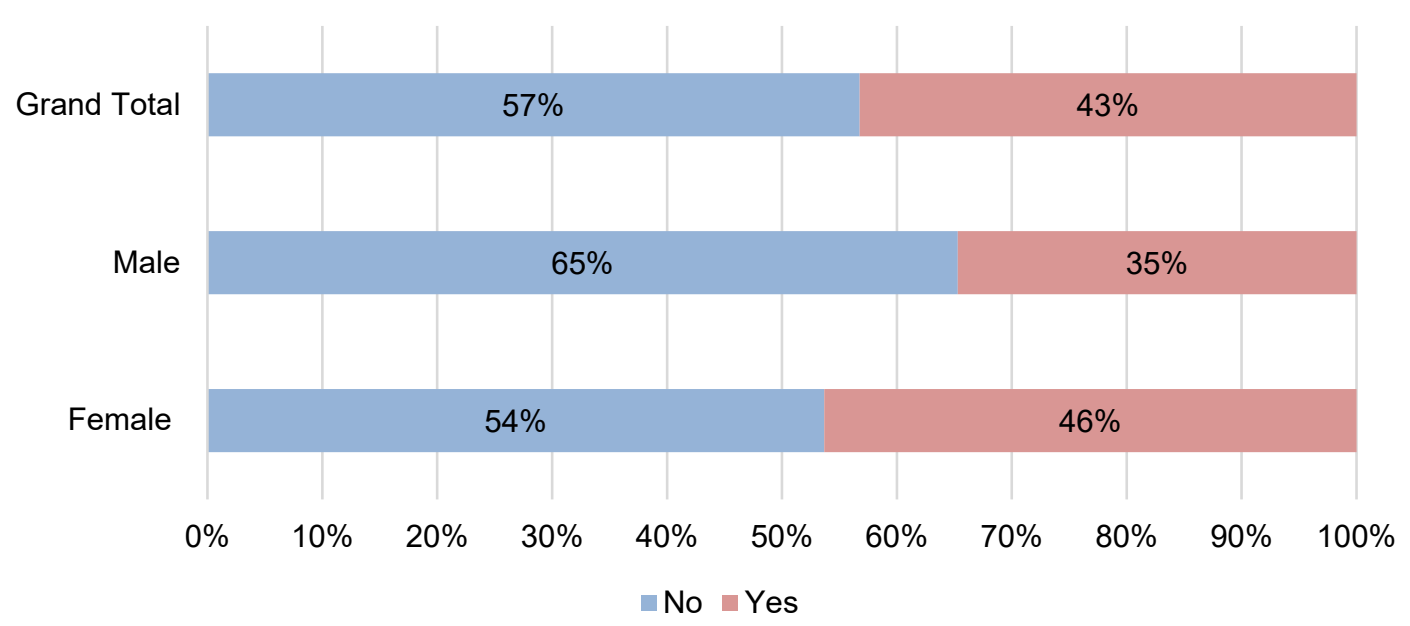

Figure 29: Reasons for a lack of access to safe bathing

If no access to bathing, what are the reasons?

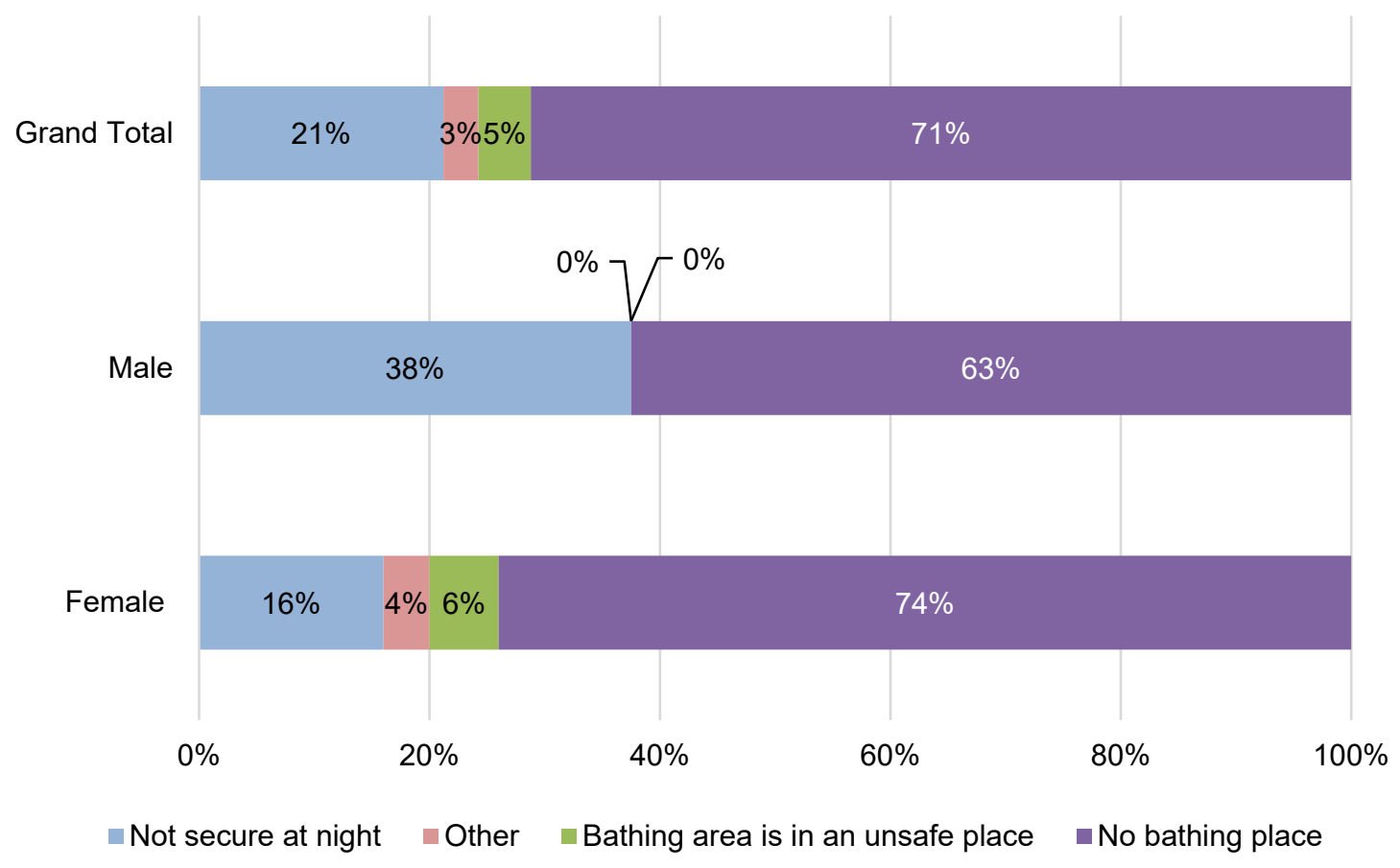

There are few indications that men and women in the communities have participated in consultations. Around $86 \%$ of men and $63 \%$ of women said they had not been consulted on the location of the water point ( $23 \%$ of women mentioned that the water point had not been installed by an NGO) as per Figure 30 . This was further confirmed by KIls across the communities. 
Figure 30: Consultation on water point location

Were you consulted on the water point location?

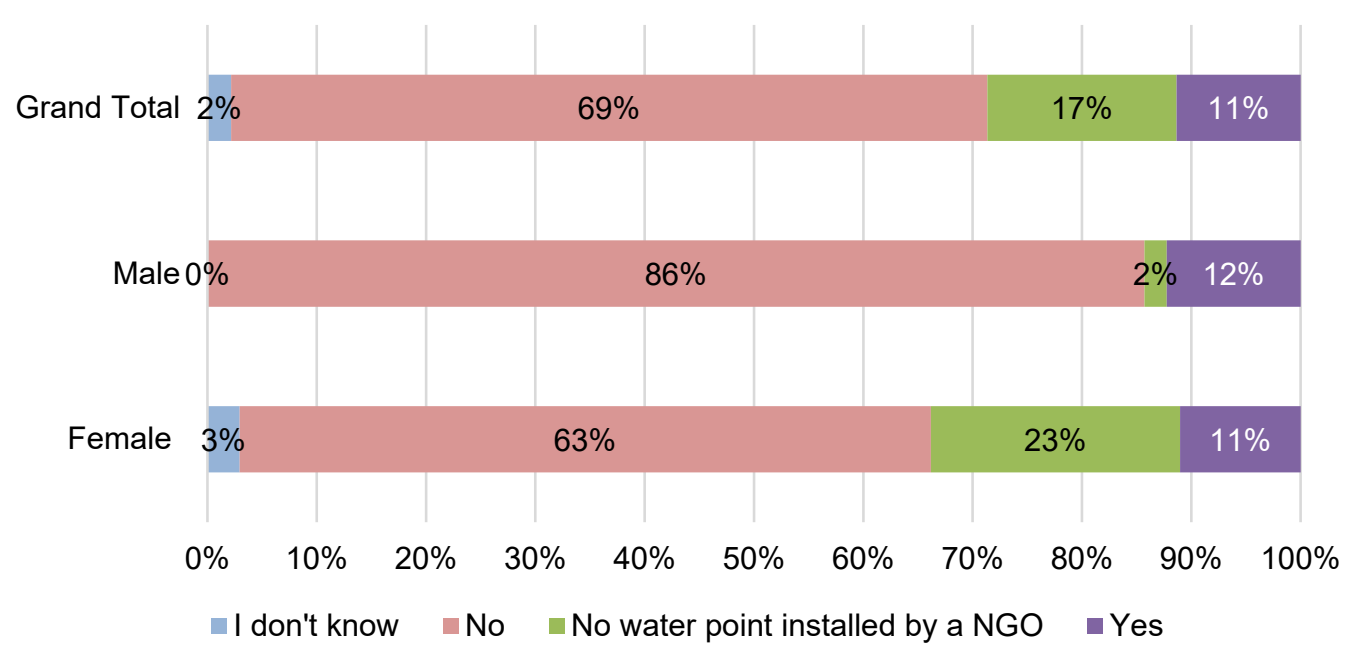

On latrine location (Figure 31) and bathing location (Figure 32), both men and women in the community responded that they were not consulted.

Figure 31: Consultation on latrine location

Were you consulted on the latrine location?

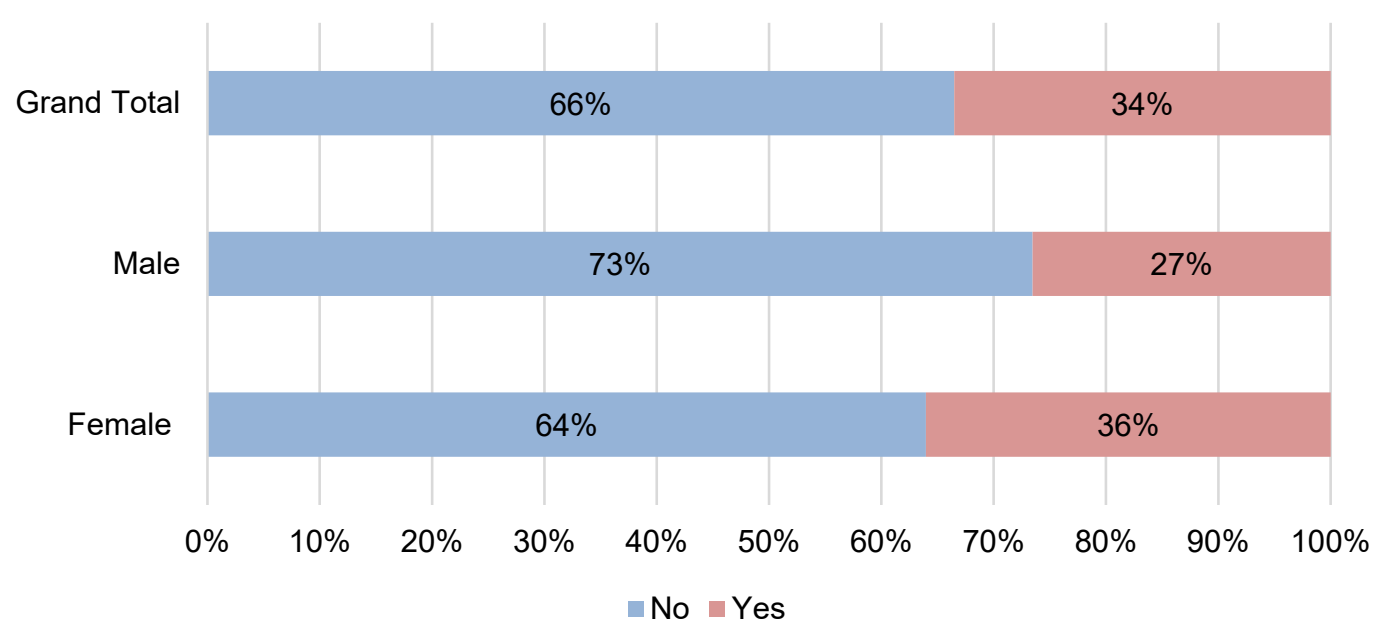


Figure 32: Consultation on bathing location

Were you consulted on the bathing location?

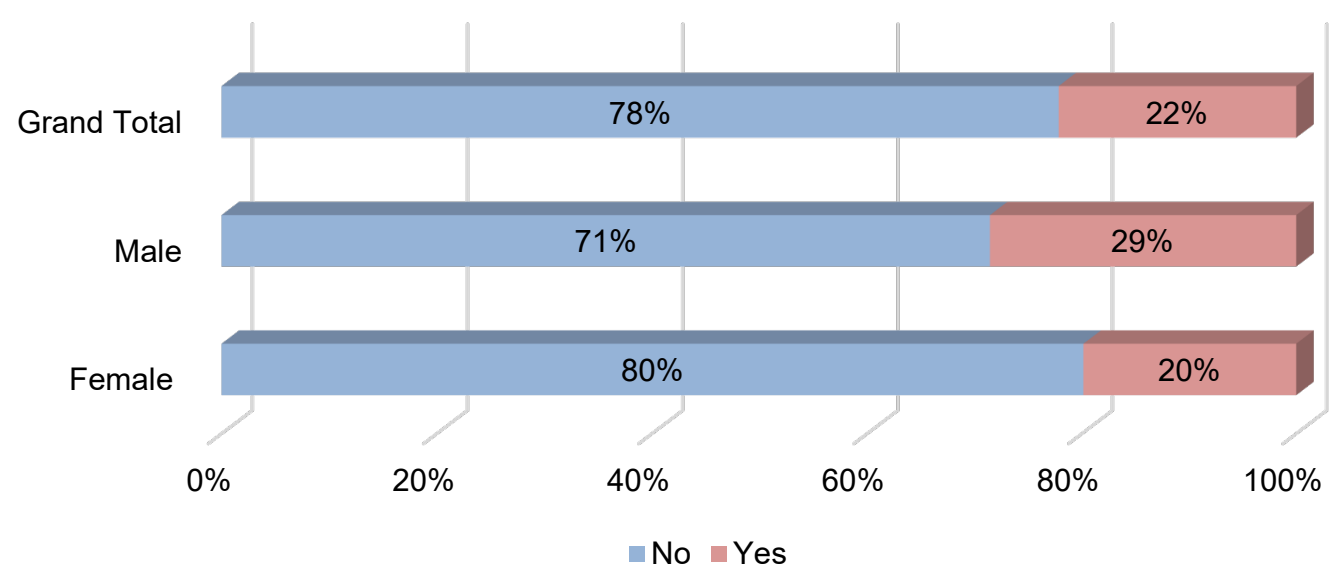

For both men and women, soap was one of top three items most needed in the household, the other two items being blankets and clothing (Figure 33).

Figure 33: Top three most-needed items

What three items are most needed in your household?

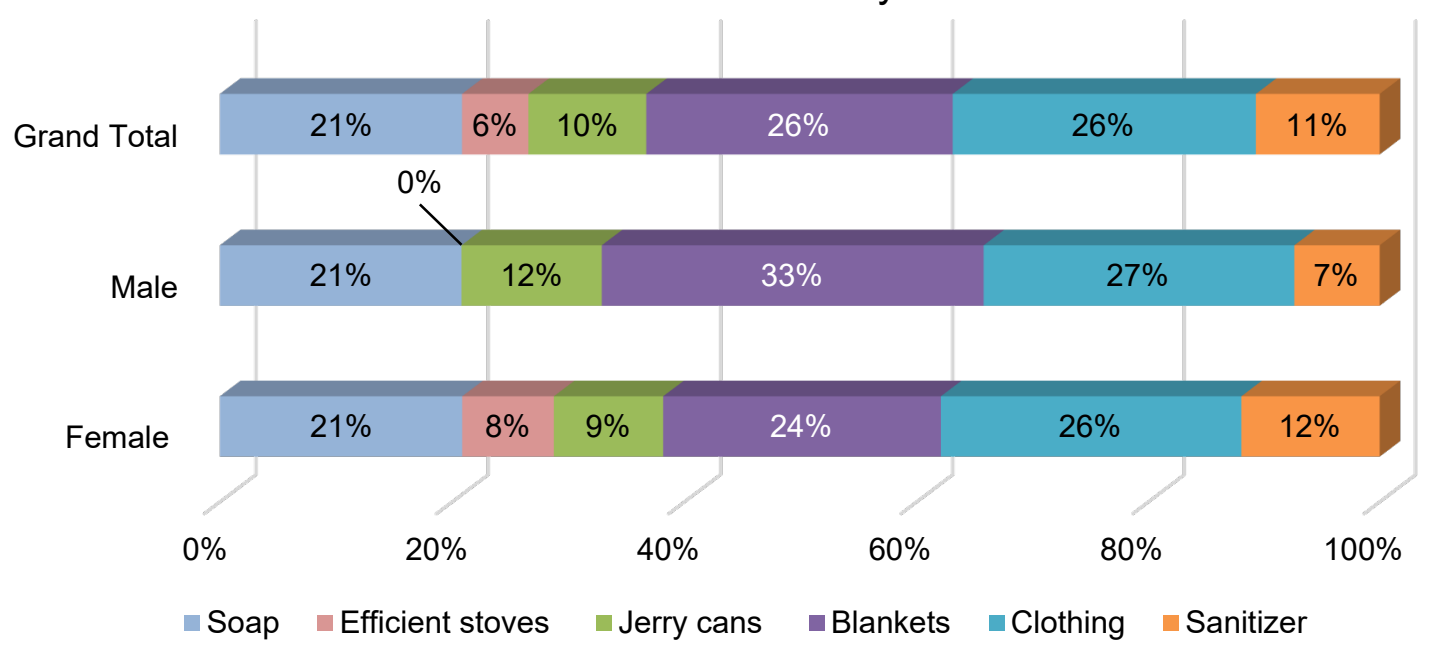




\subsection{MENSTRUAL HYGIENE MANAGEMENT}

The FGDs confirmed that there was a gap in the access to proper menstrual hygiene management for the women and girls in these communities. If supplies for menstruation are available, they are costly. Private spaces for proper cleaning practices do not exist and the locations of toilets and water sources are inconvenient.

In the survey, however, when asked whether their menstrual hygiene needs are being met, $72 \%$ of women said they were, compared with $27.9 \%$ who said they were not (Figure 34 ). This difference could be due to the type of data collection, with FGDs allowing for more detailed discussions beyond a simple yes or no answer.

Figure 34: Whether menstrual hygiene needs are met

Are your menstrual hygiene needs being met?

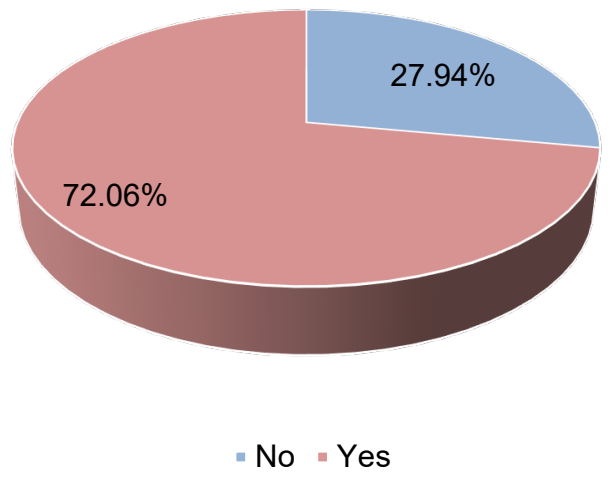

In the FGDs, pads were said to be the most common technique of dealing with monthly periods, due to the fact that many respondents had access to pads provided as part of humanitarian aid (Figure 37). However, pads are not always available and if they are, they are extremely expensive. For example, in Bariga Bosaaso, girls and women are often faced with the reality of not being able to buy pads or Bilan (a disposable sanitary kit manufactured by Dauus) every period cycle, and a lack of underwear poses a further issue with pad use. The women and girls do not have basic hygiene supplies such as soap and shampoo and resort to using ash and clay instead to wash. The IDP women in Bariga Bosaaso use cotton cloths to maintain menstrual hygiene. The girls receive sanitation kits from KAALO or sometimes are able to buy pads, or else resort to the traditionally used cotton cloths.

In the survey, however, almost $40 \%$ of women preferred pads and $56 \%$ preferred reusable cloths (Figure 35). Again, these differences can be due to the type of data collection, and more consultation with women and girls around menstrual hygiene management is needed before aid agencies and organizations decide on the type supplies that are distributed. 
Figure 35: Current preferred practices for menstrual hygiene

\section{What are your preferred practices for menstrual hygiene now?}

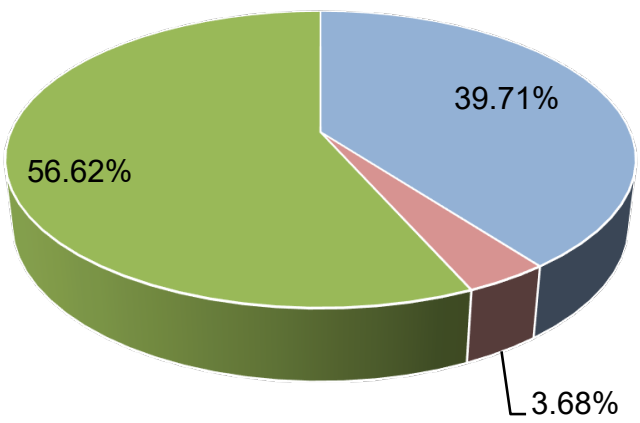

= Disposable sanitary pads $=$ Other $\quad$ - Reusable menstrual cloths

In Jowle, where the products cost almost \$2, and, for girls especially, it is hard to get money for pads, cloth material is used for menstrual hygiene, and this lack of options is perhaps why it was given as a preferred choice in the survey. Dignity kits have been distributed in Jowle, but most women and girls do not have access to them as there are never enough. A traditional belief shared in the FGD with girls in Jowle is that menstruating women and girls are told not to touch children when they are on their period; otherwise, that child will suffer mental disorders or head injuries.

In terms of disposal, the survey responses show that the largest number (41\%) use reusable items that they wash and dry, followed by disposal - equally split between bins, burying, and putting them in latrines (Figure 36).

Figure 36: Disposal methods for menstrual hygiene materials

Where do you dispose of menstrual hygiene material?

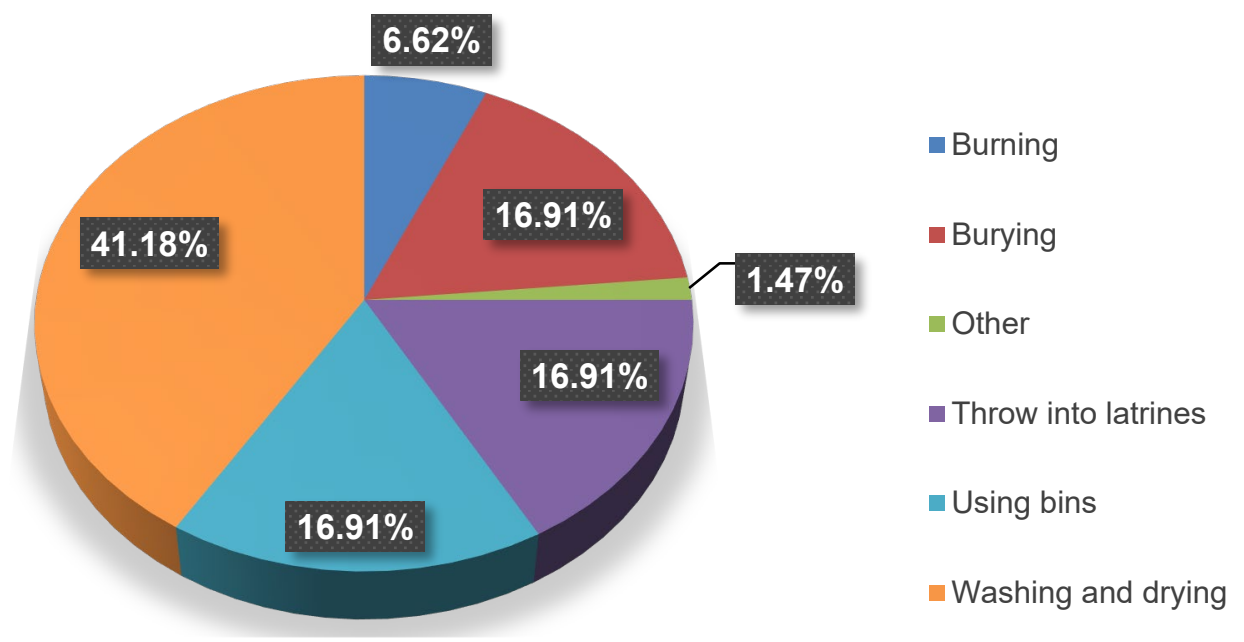


Girls in Al Khayraat use pads for menstrual hygiene, disposing of the pads using toilets and garbage tanks. They also noted the use of traditional methods such as cloths and dispose of those using rubbish bags. They said that the distributed menstrual hygiene kits are not enough, and commercial products cost too much. Occasionally girls get sick when on their period and cannot afford any medicine.

In the survey, the largest portion of respondents (41\%) said that they access menstrual hygiene materials from humanitarian agencies (Figure 37). Figure 37 also shows why most of the women said that their hygiene needs are met, with $29 \%$ able buy products from shops and a further $18 \%$ saying that they make them themselves. Nonetheless, distribution of adequate and sufficient dignity kits is very important, and dignity kits were specifically requested in the FGDs, especially by girls. If they are reusable items, it is also important to ensure access to soap and safe water for hygiene maintenance.

\title{
Figure 37: How respondents access menstrual hygiene materials
}

How do you access menstrual hygiene materials now?

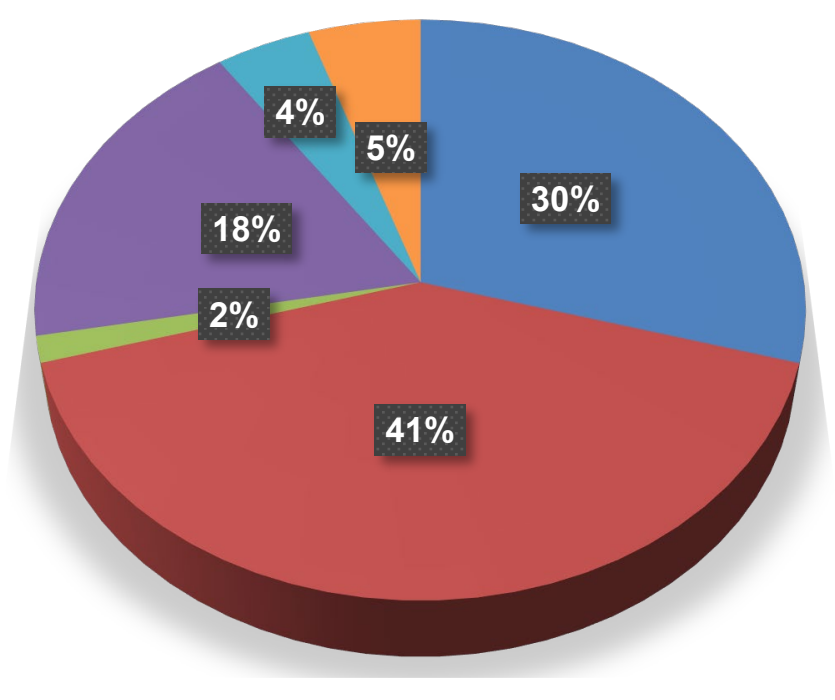

\author{
- Buy in shops \\ Distribution from humanitarian \\ agencies \\ - I cannot access menstrual \\ hygiene material \\ - Make them ourselves \\ - Other \\ - Use cloths or local management \\ way
}

\section{ACCESS TO FOOD SECURITY AND LIVELIHOODS}

It was evident across the FGDs, KIls, and survey data that access to secure and steady livelihood opportunities in the communities is minimal. Manual labour jobs that men initially would have been hired for, such as in construction, are now seeing an increased preference for foreign workers. It becomes a resounding trifecta of no opportunity, no income, and no means to support oneself or one's family. 


\subsection{FOOD SECURITY AND LIVELIHOODS}

In terms of gendered livelihoods, for the Bulo Migis, Bariga Bosaaso, Al Khayraat, and Jowle (IDP) communities, all the family members work in casual labour activities within the camp. Men undertake fishing (in the coastal areas), pottery, building, carpentry, and other types of hard labour. Women work in cleaning services, either for households or laundry services. Some collect garbage for money (taking garbage from homes to the assigned dumping ground), while others said they collect empty water bottles and exchange them for money or food (they go around the cities and cafeterias, collect empty water bottles for recycling, and take them to the local factories). Women also sell products in the market such as vegetables and jewellery. Girls do similar work as their mothers, while boys work mainly in fishing (in the coastal areas) and shoe polishing. For the host communities of Kalabayr, Carta, Geri Hel, Budunbuto, Cuun, Kubo, and Saacada, livestock, farming and the export of frankincense were noted as their primary sources of income. Women in Cuun collect caw, a natural material from which they make mats to sell.

In terms of food security, coping with food shortages has become all too common and families are adjusting their consumption by reducing their daily intake, eating just once or twice a day. Women in Bulo Migis noted that no improvements in food security are being made in the community. Men are worried as they are unable to provide groceries for their families.

In Bariga Bosaaso, women reduce the amount of food being cooked and the number of meals per day, while in Jowle, meals are served once a day. There are food shortages, and that which is available is expensive and many cannot afford to buy it. There is no financial assistance or job opportunities to help secure and stabilize basic household needs. The IDP men in Jowle restated the food shortage and said that even with what is available, low wages and high food prices leave few options. There is no meat, only rice and vegetables. IDP men in Al Khayraat and Bulo Migis also mentioned that men are paid for their work in the local currency, but that merchants would only accept US dollars. Due to high inflation and the unstable exchange rate, the government had shifted to not collecting taxes in the local currency, and using US dollars is expected for official government transactions. All four men's FGDs (in Bulo Migis, Bariga Bosaaso, Jowle, and Al Khayraat) acknowledged they are the ones that have the responsibility for securing goods for the family.

Men in the host communities in Kalabayr, Saacada, Carta, Kubo, Geri Hel, Cuun, and Budunbuto highlighted the devastating effects of droughts, floods, and locusts, where the crises wreaked havoc on farms, livestock, pastures, and water sources. According to the men in Carta, when the livestock died, so did their livelihoods.

The survey results reveal that half of the respondents ( $49 \%$ of men and $57 \%$ of women) do not have access to a source of income (Figure 38 ) and only $39 \%$ of the men and even fewer women (24\%) said that their source of income was stable (Figure 39). 
Figure 38: Family source of income

Does your family have a source of income?

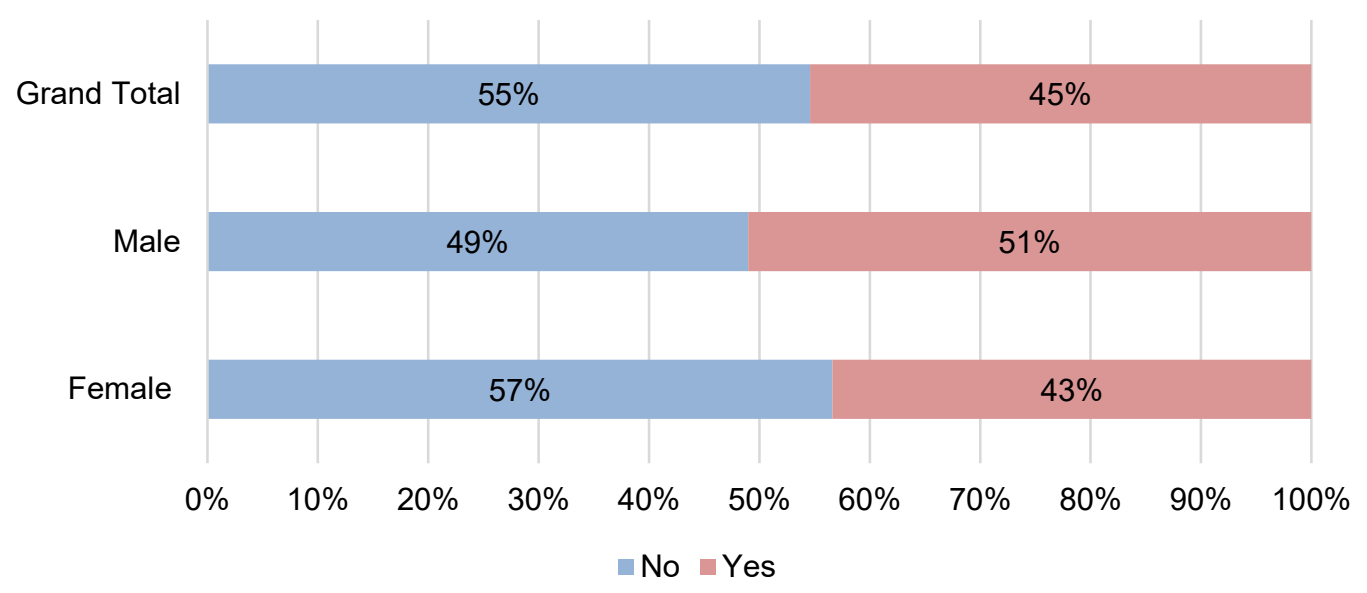

Figure 39: Stability of income sources

Do you have a stable source of income?

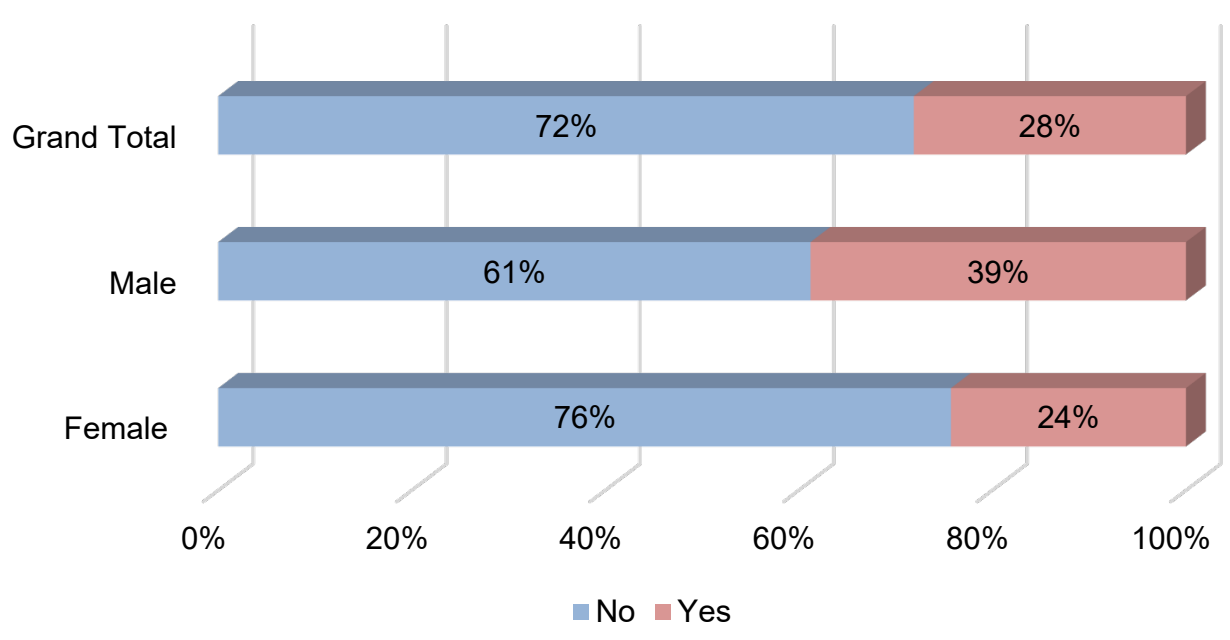

The survey results also reveal that $61 \%$ of men and $57 \%$ of women do not have enough food to meet their family's needs (Figure 40 ) and that only $25 \%$ of men and even fewer women (14\%) receive any cash assistance (Figure 41). 
Figure 40: Whether respondents have enough food to meet the needs of the family

Do you have enough food to meet the needs of the family?

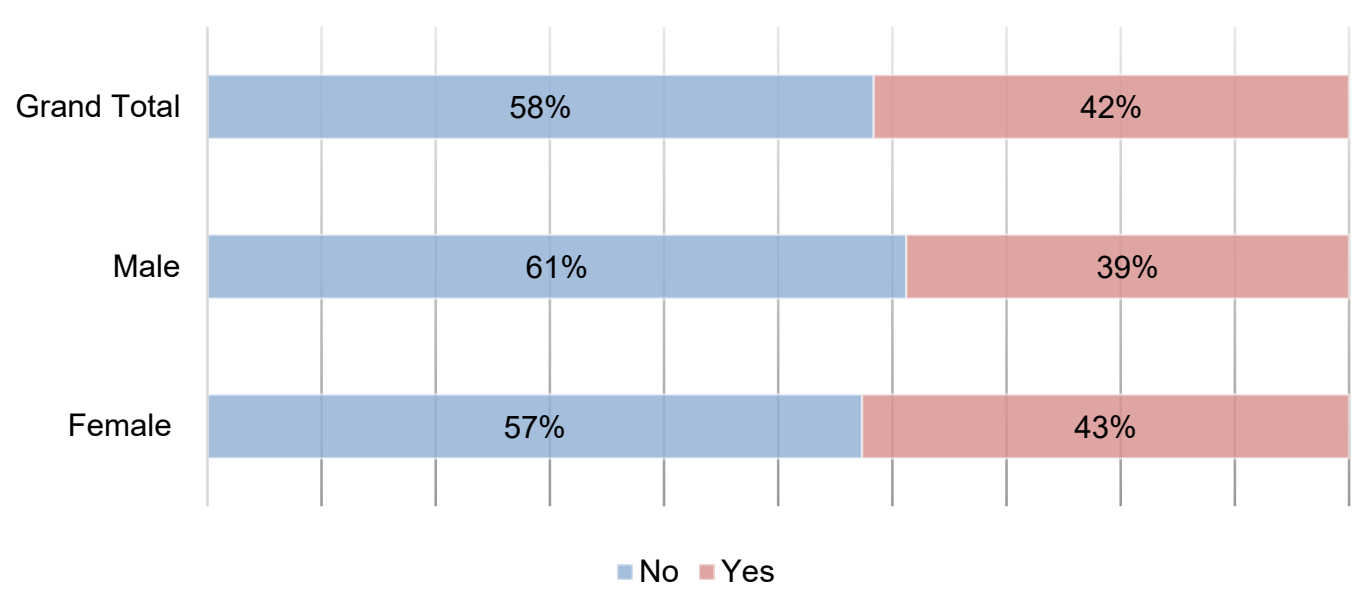

Figure 41: Whether respondents receive cash assistance

Do you receive cash assistance?

No $\square$ Yes

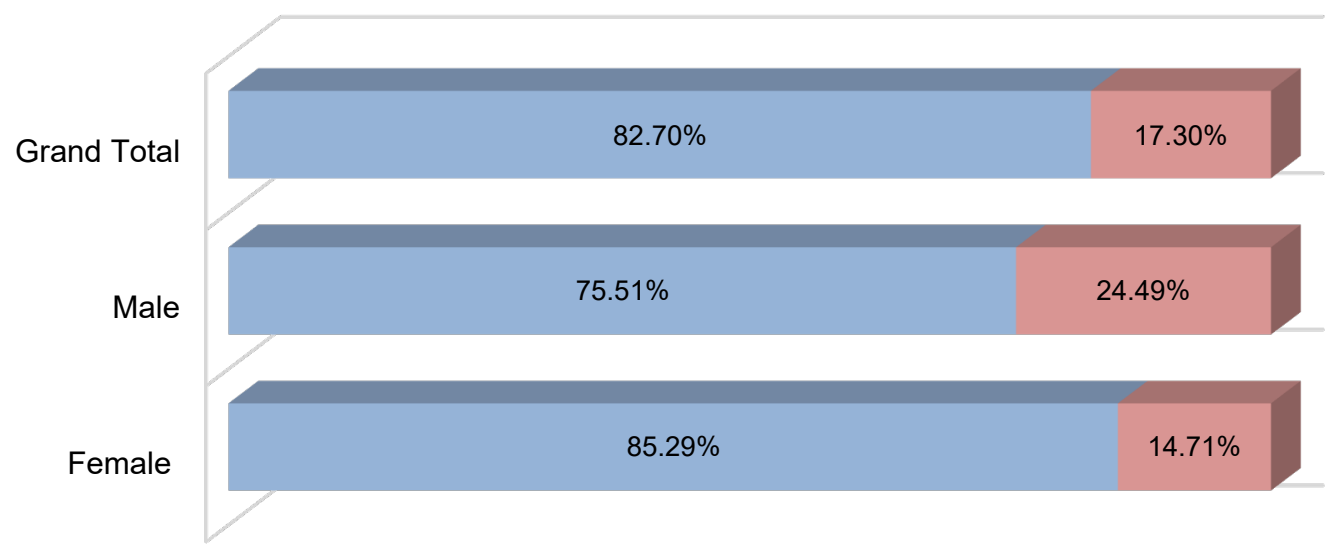

It is also important to note that the survey shows the effects of the crises on livelihoods $-92 \%$ of men and $58 \%$ of women said that the current crises affected their livelihood options (Figure 42). When women were asked separately whether they were able to carry out any economic activity, $59 \%$ said they were not (Figure 43). Further, respondents in the FGDs mentioned that they were primarily suffering from monetary inflation. With decreases in the street value of the Somali shilling for the past year, the average person makes about \$2.50-\$3 per day. In addition, the reduction in work opportunities associated with inflation has added another layer of economic strain. Furthermore, the reduction in value has resulted in the need to carry large amounts of paper money. Those doing so in public, especially at night when workers head home, regardless of their sex, are at an increased risk of theft and violence.

On top of the effects of inflation, the effect of the COVID-19 lockdown on livelihoods was overwhelmingly evident from the answers of both men and women (Figure 44). Across all locations, community KIls 
confirmed that the COVID-19 measures have increased economic stress, with one saying they have been 'more harmful than helpful', damaging women's small businesses and resulting in lost job opportunities for men. Livestock, and particularly camels in Budunbuto, Cuun, and Geri Hel, were reported as suffering from unknown diseases. The communities assume that this is a result of eating the leftover pasture eaten by locusts, and some said that COVID-19 was also affecting livestock. In Budunbuto, the host FGD said that the quality of milk was different, and children became sick whenever they drank the camel and livestock milk. A key informant in Geri Hel added that livestock cannot be sold because of the lockdown measures and that as the livestock got sick, the fee for livestock to be taken to the city doubled and was thus unaffordable. This was confirmed by other key informants. Rickshaw prices have gone up because of social distancing, with fewer people per ride having to pay more.

Figure 42: Whether the current crisis has affected livelihood options

Has the current crisis affected your livelihood options?

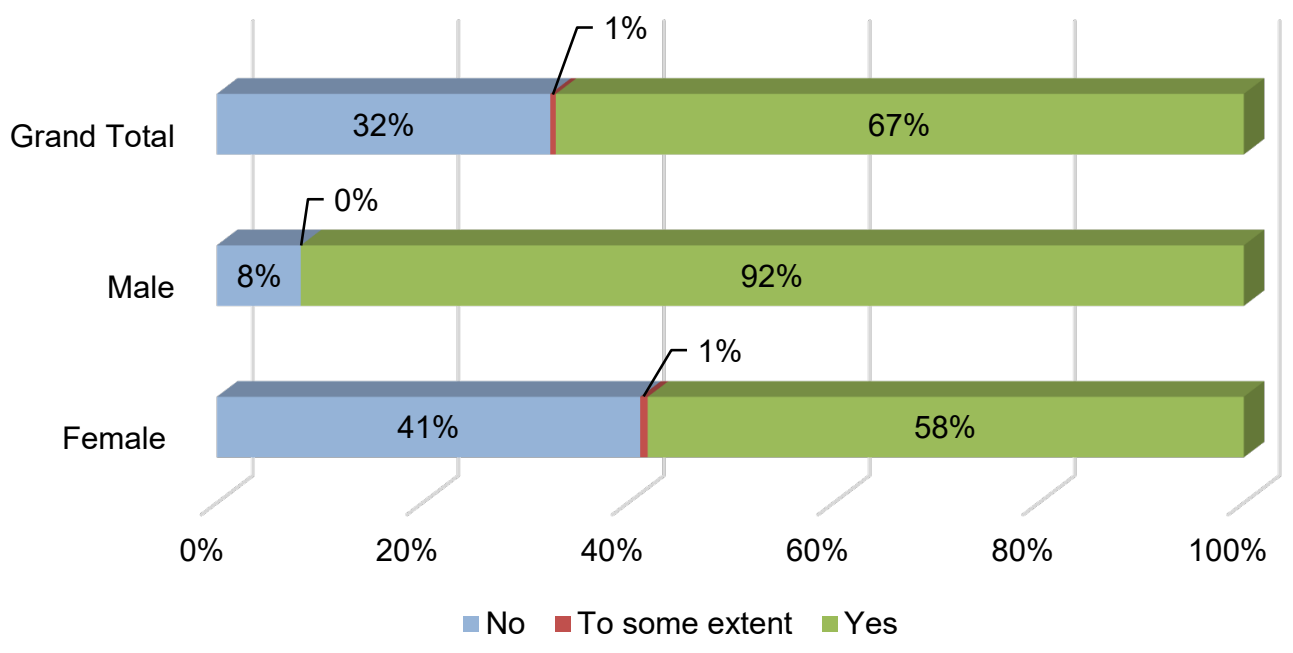

Figure 43: Whether women were able to carry out economic activity after the crisis

Women only: were you able to carry out an economic activity after the crisis?

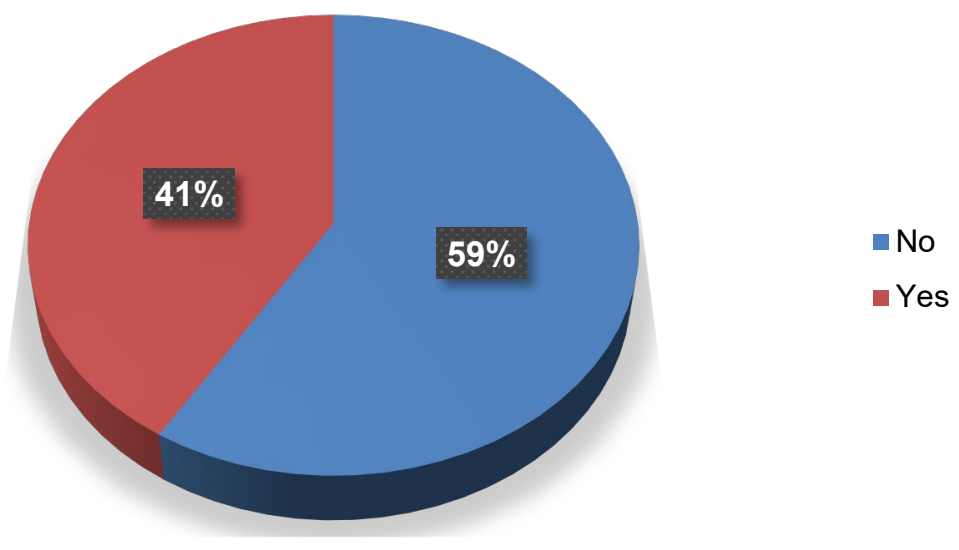


Figure 44: Whether lockdown affected livelihoods

Did the lockdown affect your livelihoods?

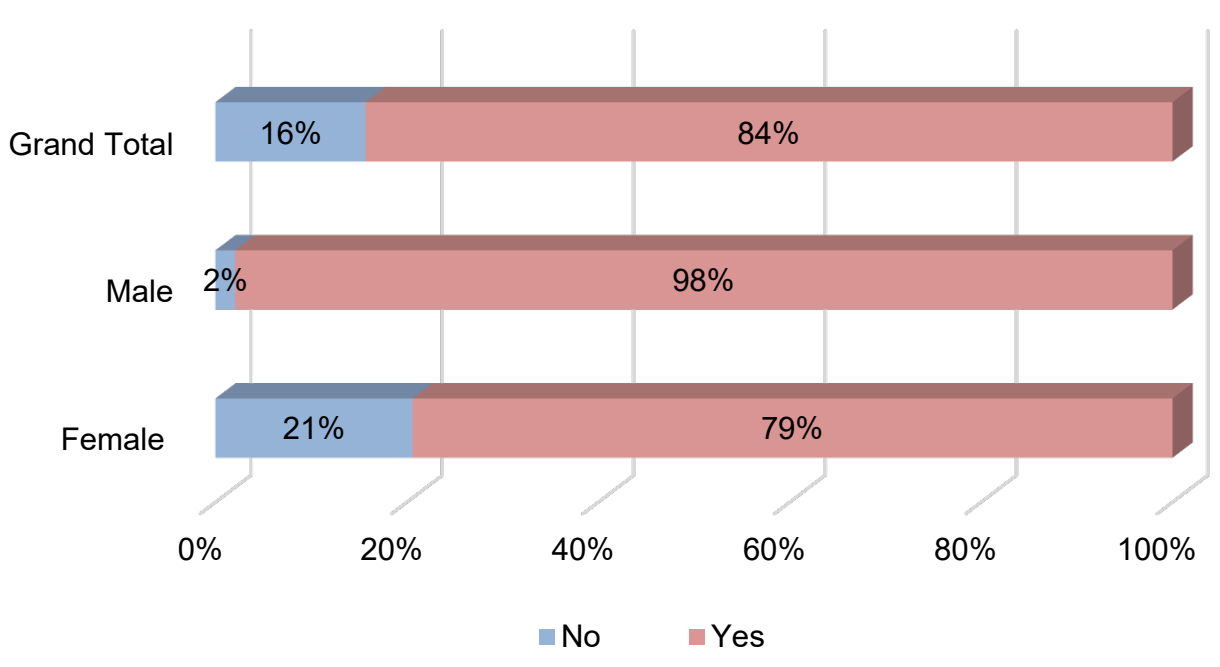

In addition to COVID-19 effects, in discussions with host community women, the ripple effects of multiple crises were clear. In Geri Hel, there is no livestock - no meat, no farm production, no milk, and no food. In Carta, women explained that currently there are no vegetables. For the women in Kalabayr, livestock sales were weakened by droughts and COVID-19, in addition to locust infestations which caused food scarcity and elevated prices. In both Carta and Kalabayr, their previous primary income source, exporting frankincense, had to be suspended due to COVID-19. This is a business in which mostly women work, so it has affected them disproportionately. The host community women in Cuun noted that 'life is all about sharing, we are simple farming family' and that there are serious food security issues and no livestock in the community. In Al Khayraat camp, the women do not have farms or livestock, so their only option is buying the necessary goods in the town markets. For this type of purchasing, women are responsible.

The challenges of the compounded crises were confirmed by the KIls with community leaders, who mentioned the decrease in employment for men and damage to small livelihood opportunities for women, the loss of shelter and savings, malnutrition, and inflation, specifically in the IDP camps. Overall, the crises have had a deep impact on livelihood and economic opportunities, including on farming in the host community. The KIls also highlighted that some groups were more vulnerable, such as women and girls from minority clans, female household heads, pregnant women, elderly people, or people living with disabilities, but they did not go into detail about the specific needs of each group.

On the changes brought about by the crises, the men's IDP FGD in Bariga Bosaaso noted that women and girls were previously housewives, farmers, and livestock herders (of sheep and goats), and in the current crises they do work such as washing clothes, street sweeping, or cleaning for other families. The Bariga Bosaaso FGD saw women's role in the household before the crises as just being housewives, and though some of the men said women used to work, most said that the women never contributed financially previously. According to the Bulo Migis men's IDP FGD, although women spent most of the time inside the household doing childcare, they were also engaged with milking, and processing milk and meat products. The Bulo Migis FGD further observed that before displacement from the south, men were involved in farming, livestock herding, and some were even teachers. After displacement, men became casual labourers, drivers, car washers, and construction workers. This work, they observed, was done at lower wages than the host population. 
It is important to note women's care burden in relation to decision making around EFSVL. Women are the ones responsible for cooking, cleaning, childcare, WASH-related tasks, as well as, for some women, either supporting on or being directly responsible for bringing in income. Figure 45 shows that $69 \%$ of men and $43 \%$ of the women said it was the woman who was involved in income-generating activities.

Figure 45: Who in the household is involved in income-generating activities

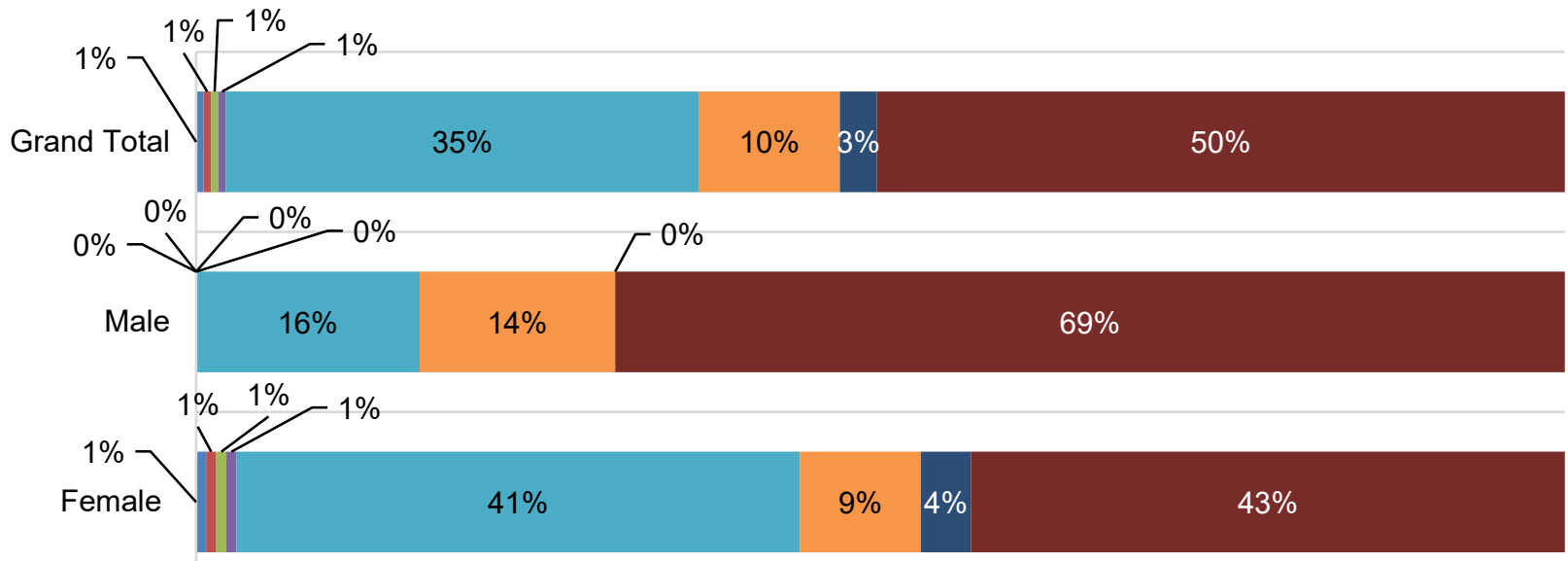

Another female member of the household

Boy

- Man

- Shared responsibility between wife and husband
Another male member of the household

- Girl

No one is involved at the moment

- Woman

It was also the woman's responsibility to do the grocery shopping, according to almost all men and women (Figure 46).

Figure 46: Responsibility for grocery shopping

Who buys the groceries?

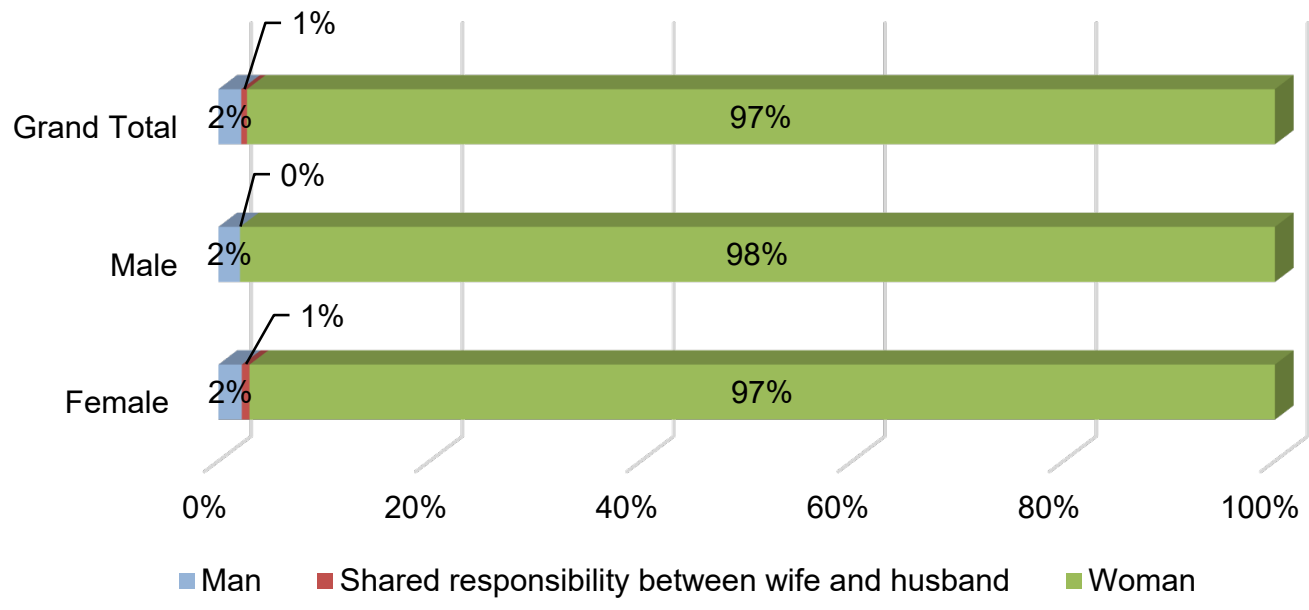


Furthermore, women are responsible for farming in the host communities, according to $67 \%$ of men and $40 \%$ of women (Figure 47 ).

Figure 47: Who is responsible for farming in host communities

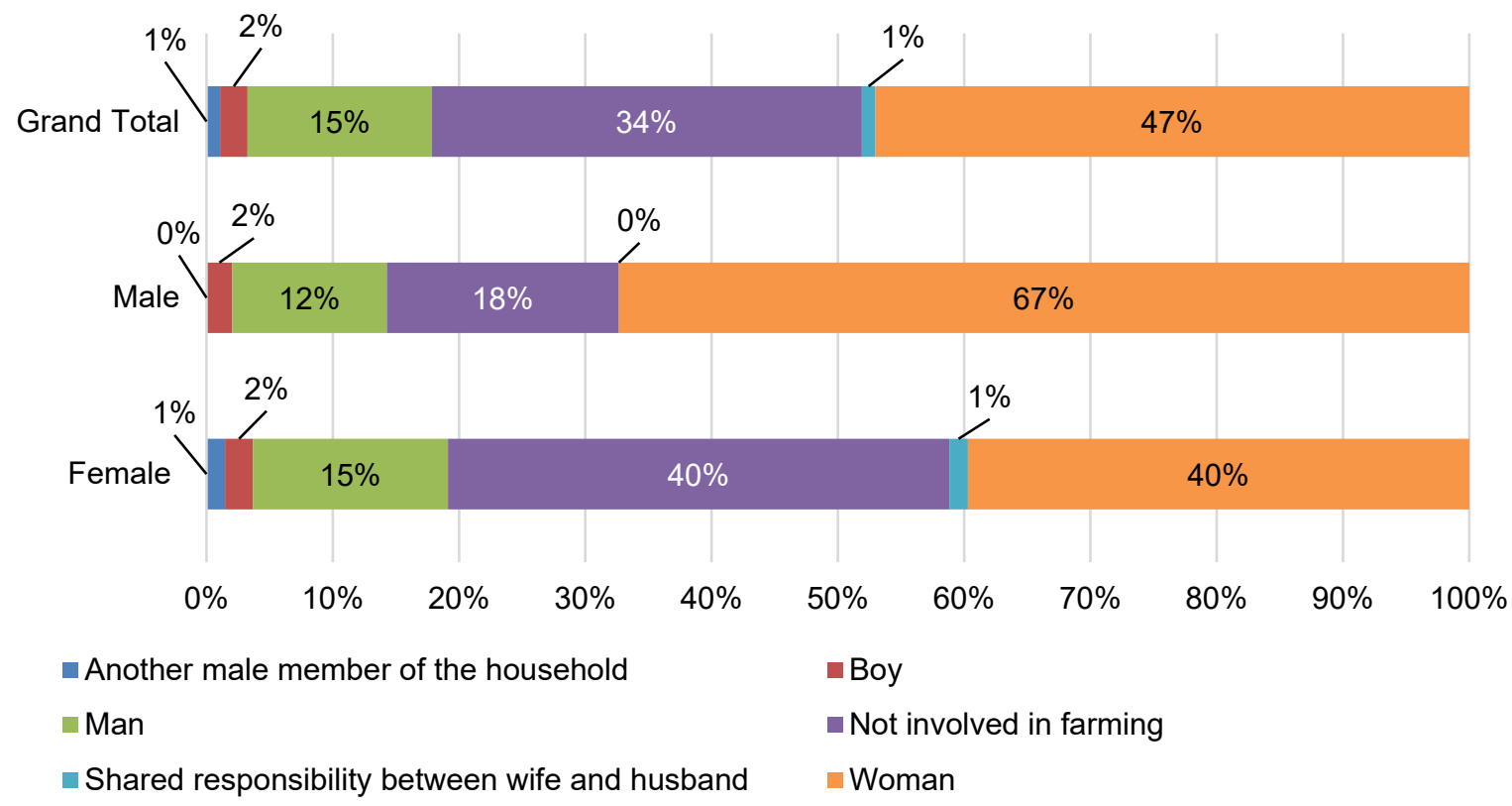

In those households that received humanitarian assistance, it is women who collect the aid, according to $76 \%$ of men and $90 \%$ of women (Figure 49 ). Figure 48 shows that just over half of the households have not received any assistance.

Figure 48: Humanitarian assistance received by households in the previous three months

Did your household receive any humanitarian assistance in the last three months?

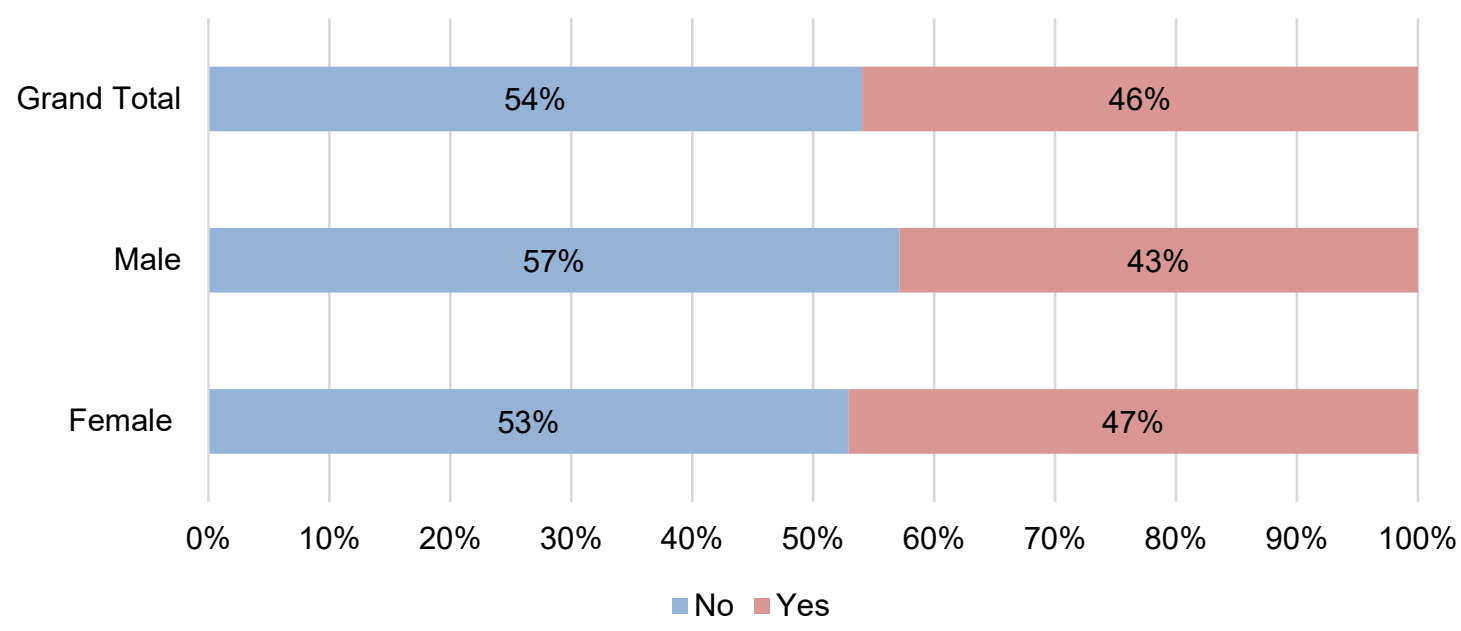


Figure 49: Who in the household collected the humanitarian assistance

If yes, who collected the assistance?

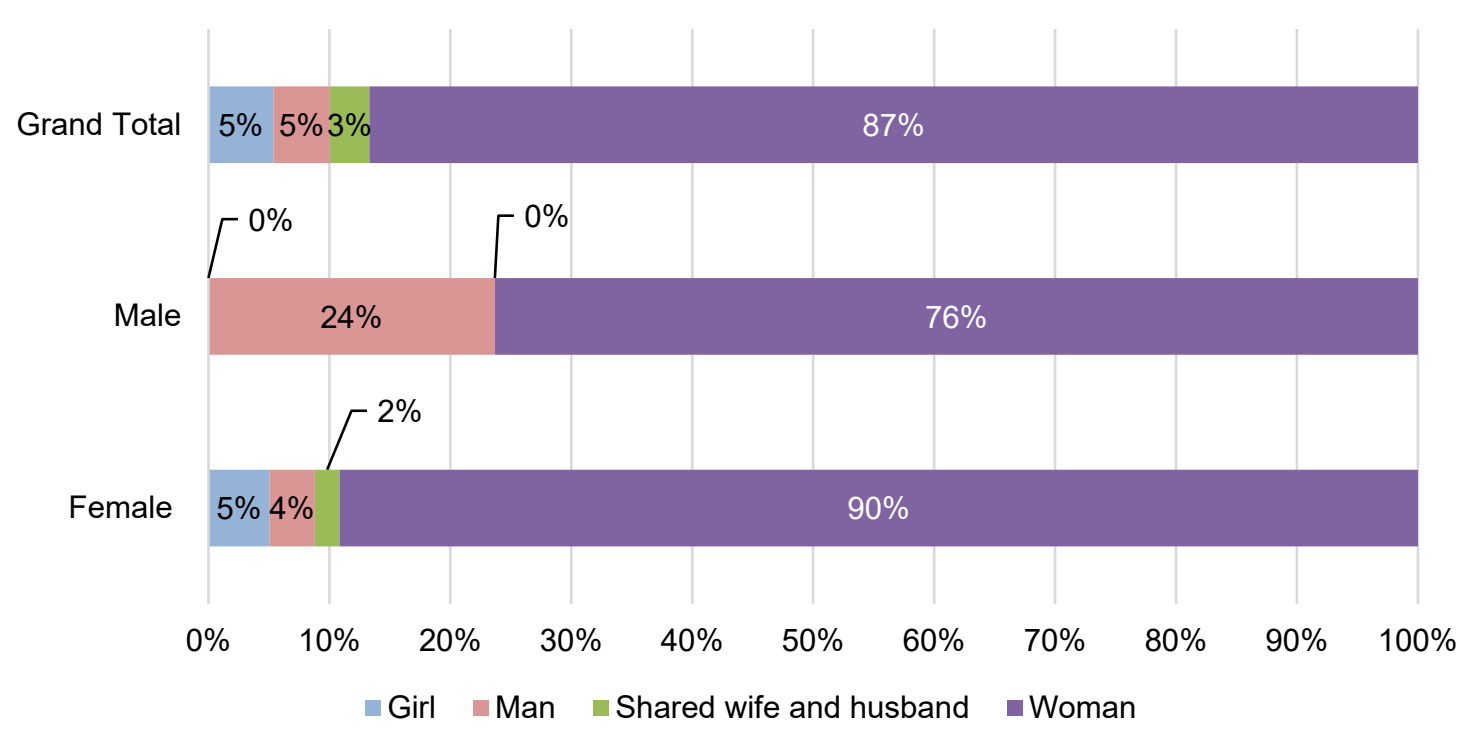

However, all decision making around household income and resources lies with the men. In relation to who decides on how to spend money, $86 \%$ of the men versus $49 \%$ of the women said it was the man (Figure 50). The large discrepancies between the answers could be due to the shift in roles as women are now earning money, but men are not yet ready to accept this situation.

Figure 50: Who in the household makes decisions on how to spend money

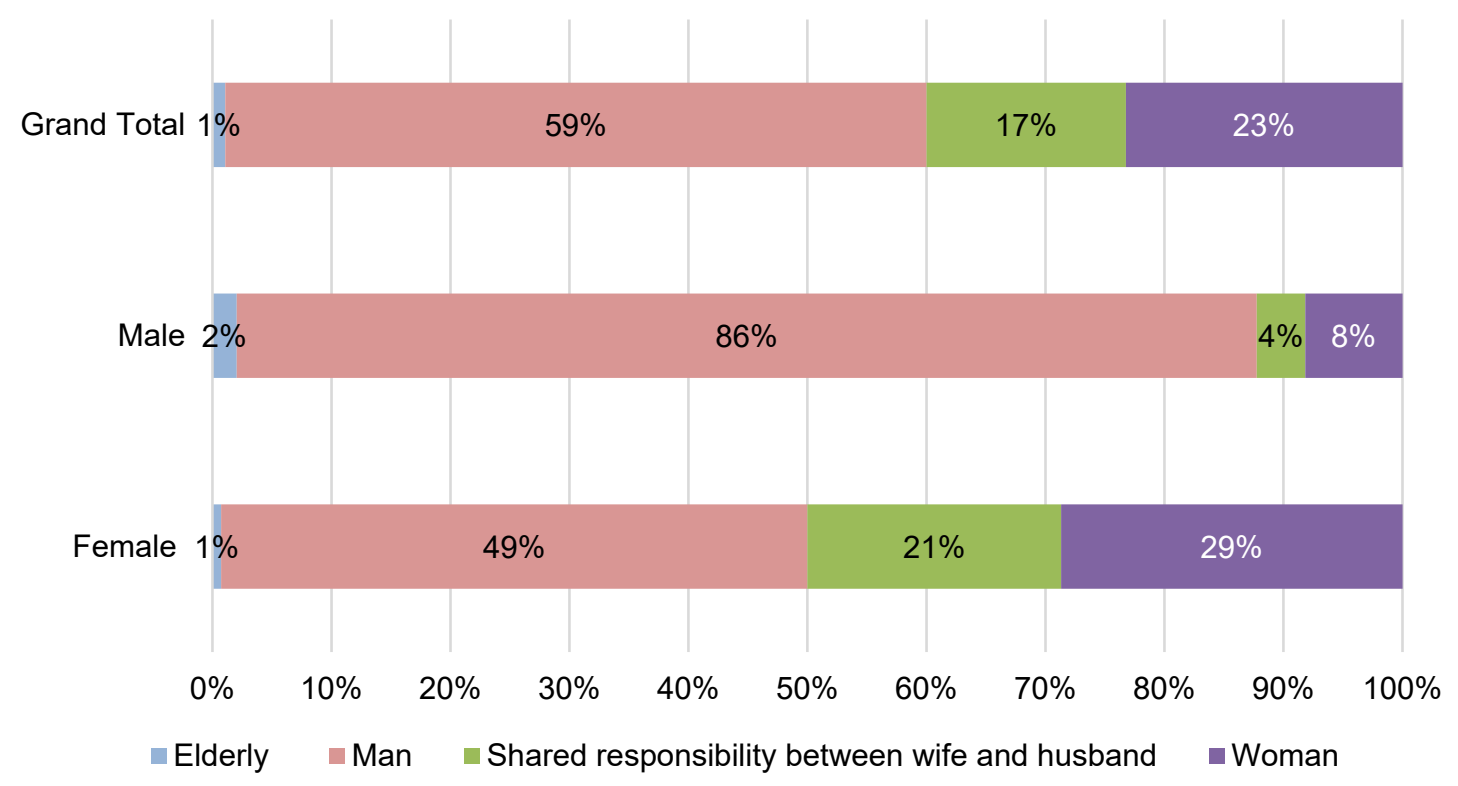

When asked if the person earning income shared it with the family, $45 \%$ of the women said no, but only $29 \%$ of men did so (Figure 51). All of these findings were confirmed by the KIls. 
Figure 51: Whether income is shared in the family

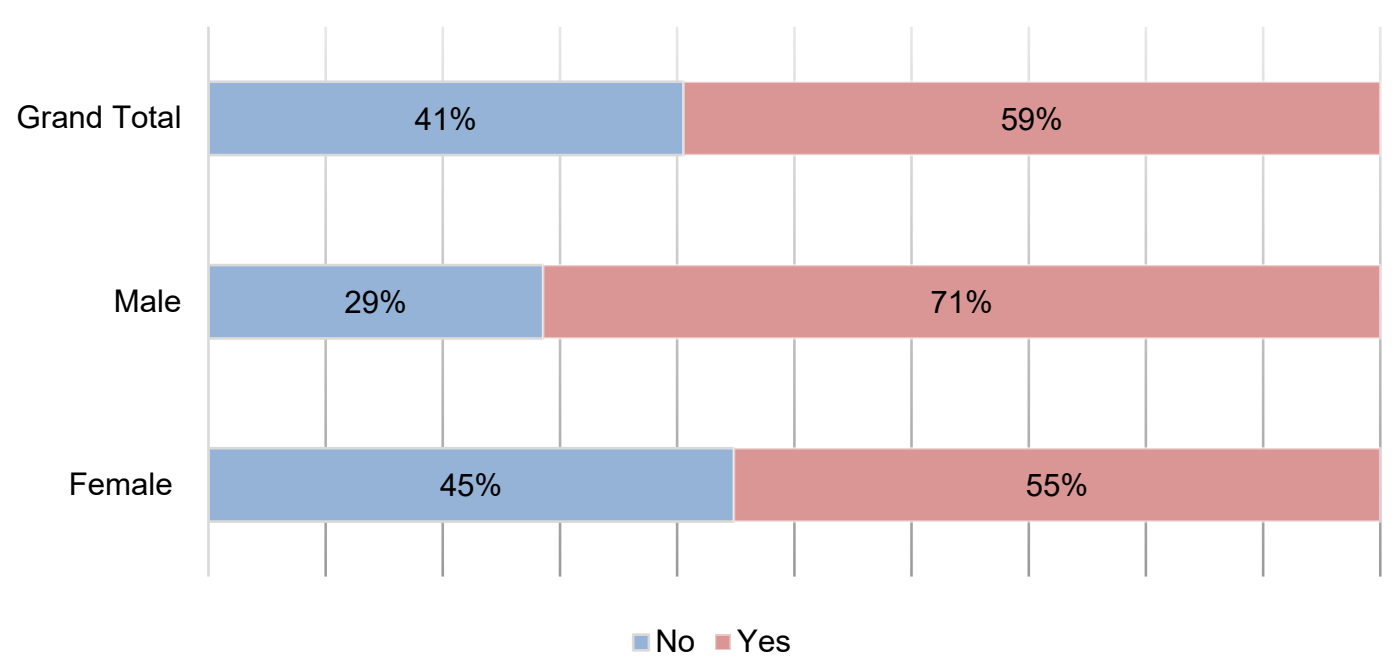

When asked who makes decisions on the household buying and selling of items, $86 \%$ of the men said it was solely them, with only $14 \%$ saying it was the woman, while $40 \%$ of women said it was solely the men, $23 \%$ suggesting that they were joint decisions, and $35 \%$ saying the women alone (Figure 52 ).

Figure 52: Who makes decisions on buying and selling items

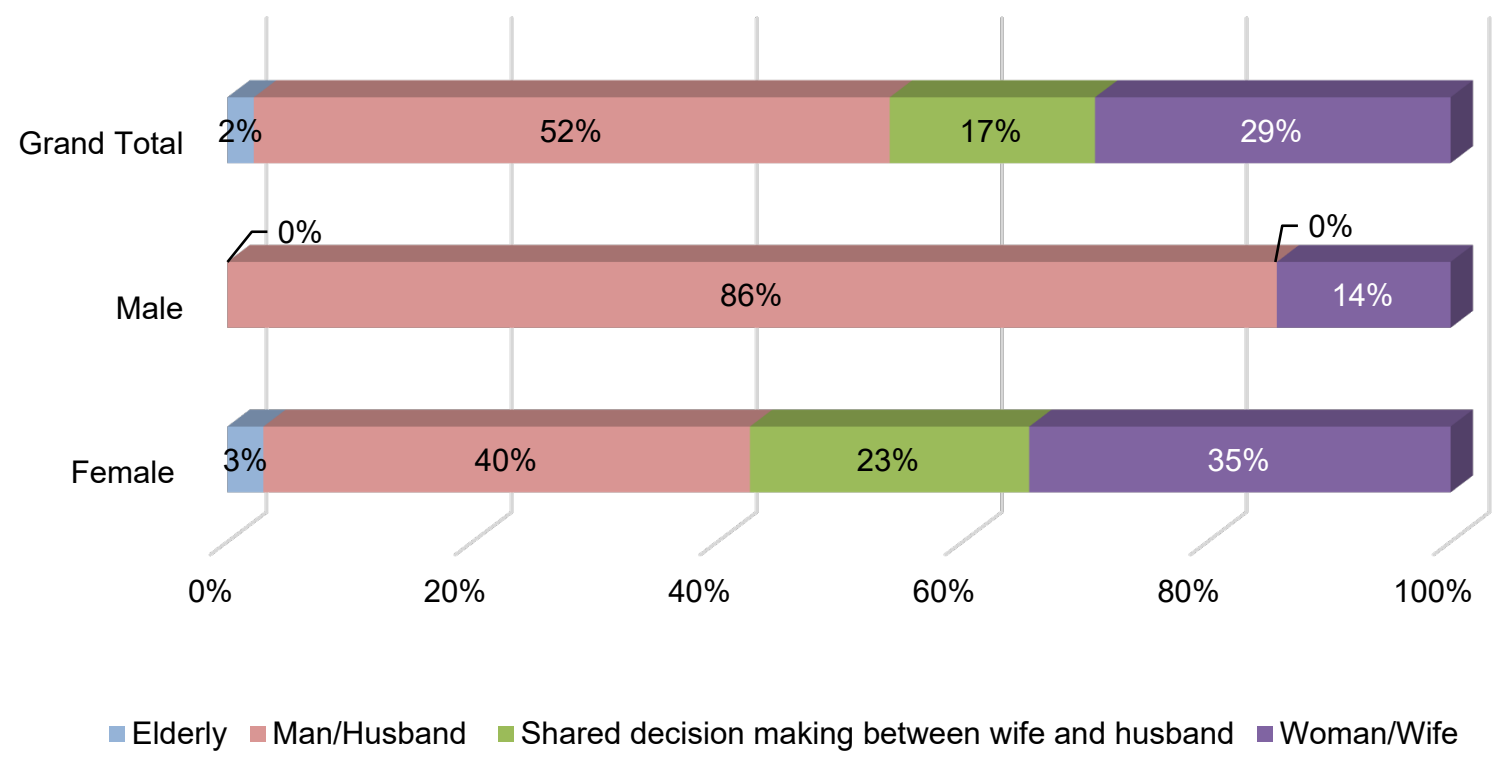

On decisions around humanitarian aid, men again responded that it was primarily them (59\%), with only $29 \%$ of the women agreeing that the men decide, and $24 \%$ saying that these are joint decisions (Figure 53). 
Figure 53: Who makes decisions on the management of humanitarian aid

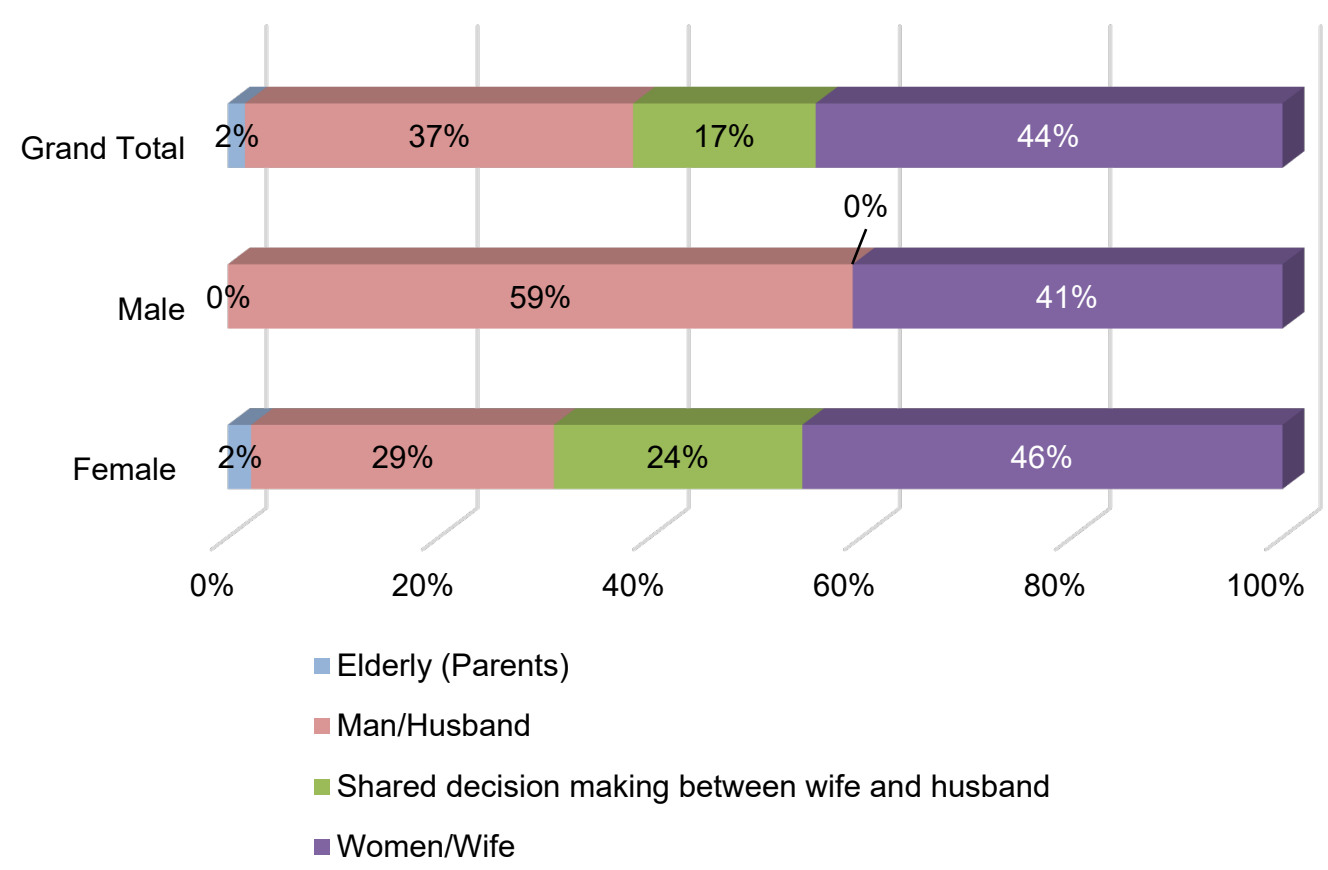

In the boy's FGD in Bariga Bosaaso, there was disagreement on women's roles in the household, as many respondents said they earn money for a living and classified that as their main role. Others said that was not their role, but that they are a member of the family and are contributing their efforts to bring in some income.

A KII with an INGO agreed that women have increasingly become household heads and main breadwinners for their families, and that gender roles have shifted, putting women in a position of increased burdens as they play double gender roles. However, this has put them at more risk as they often travel long distances in search of work, food, and water. In male-headed households, women's risk of IPV has increased as they are expected to do more than their traditional gender roles, including contributing to family income, and diminished incomes have led to heightened tensions in the household.

\subsection{SKILLS AND CAPACITIES}

Many in the communities have an informal skill set that they have used to survive. Many people (61\% of women and $57 \%$ of men - see Figure 54 ) are illiterate, so there is a greater focus on manual labour and informal services. 
Figure 54: Literacy level of survey respondents

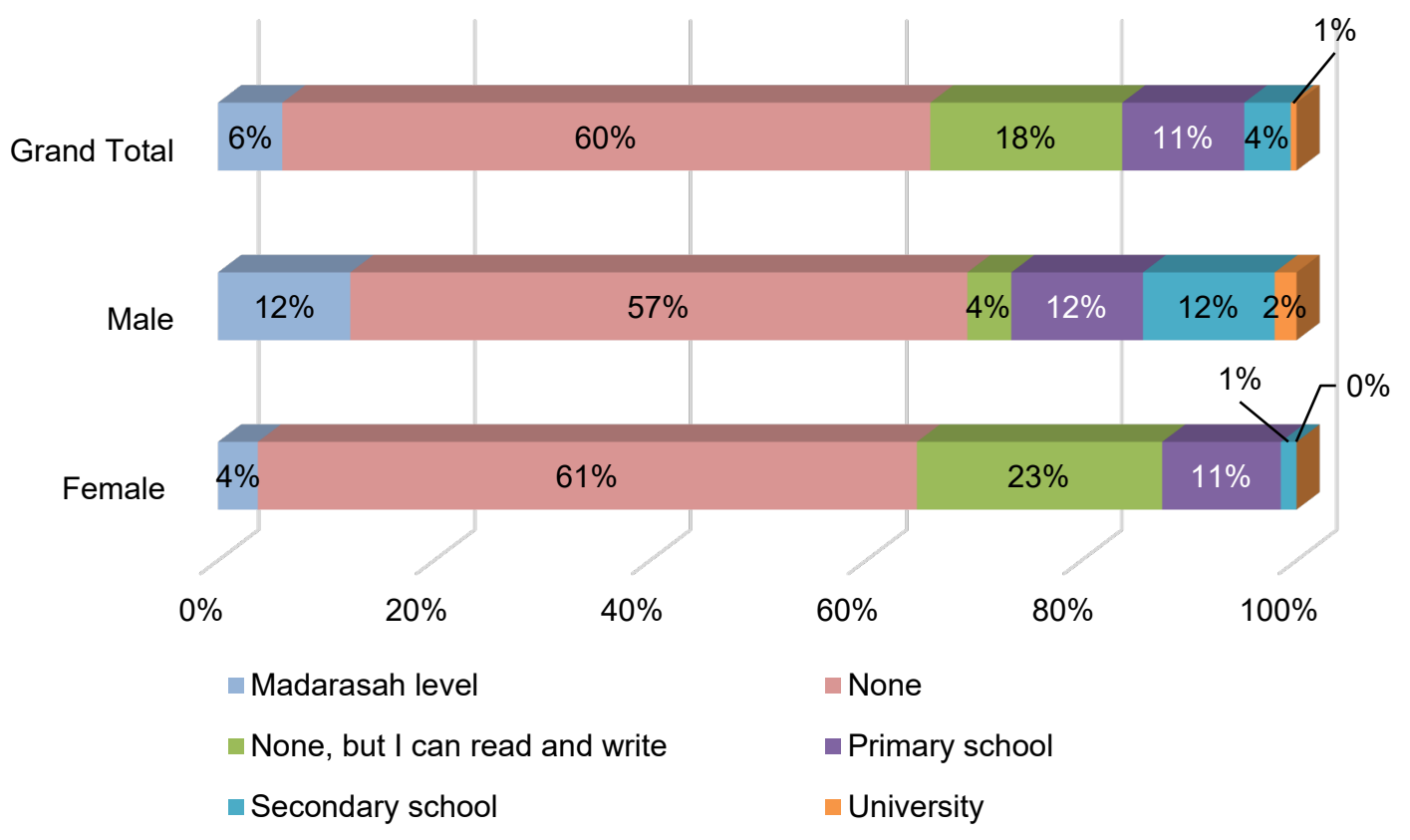

Community members across the FGDs mentioned they want to build their skills and needed free formal education to do so. They suggested that there should be schools in the IDP camps that teach Somali, mathematics, science, and art - skills that embrace creativity but that also can be a source of financial subsistence. Women, men, girls, and boys in Puntland are adept in a broad range of skills, from construction, cooking, weaving, barbering, washing cars, and body adornment (Sharaxaad), and want to expand their vocational options. In Jowle, the girls flagged that they are excluded from humanitarian aid and asked to be included in this assistance. They further requested simple machines for weaving, tailoring, and solid waste management, bodily adornment tools, training on vocational skills, and washing machines to help alleviate the excessive burden of handwashing. The girls' FGD in Bariga Bosaaso expressed the need for different types of skills and establishments, for example making paint colours, opening henna and hair salons, and access to sewing machines to make clothes. These opportunities are available but need to be capitalized on to change livelihoods in the community.

A noteworthy point made in the Carta boys' FGDs was that training is needed as well as supportive trade policies, and that COVID-19 measures needed to end to create more income-generation opportunities. Once a crisis occurs, education stops being a priority and is put on hold for families, which hampers people's prospects of building a livelihood. The Bulo Migis boys' FGD requested a specific youth programme on skills training and enhancing livelihoods.

The Kalabayr FGD recommended teaching men and boys basic skills to manage their families' livelihoods, establishing arts skills schools and formal education for boys and girls, and providing sewing machines and small business investment to empower women. In Al Khayraat, there was also a request to establish educational centres. The KIls did not mention specific skills but suggested the need for educational and capacity building training as well as a savings group for women. 


\subsection{SURVIVAL NEEDS AND COPING MECHANISMS}

High unemployment and few opportunities leave people with very limited financial resources with which to survive. Youth, both boys and girls, have had to resort to negative coping mechanisms in the current state of poverty, such as early marriage for girls, illegal migration for boys and girls, and trafficking. In Bariga Bosaaso, basic life necessities such as food, firewood, and education are things most families cannot afford. Firewood was the top need mentioned across the FGDs with communities, who suggested that the high price of firewood determined the number of meals they decided to eat a day. Further difficult decisions have to be made all the time, such as about children's schooling, and often a family can only invest in one child - preferably a boy, as noted by the Kalabayr host community women's FGD. Mothers said that they do not invest in their daughters' education because they will leave the household as soon as they reach puberty. Generally, education opportunities in the IDP communities exist mostly for boys. Similarly, host communities' schools are predominantly for boys. In Kalabayr, an FGD participant said that: "girls are [in] "transit in the household" - we try as much as we can to benefit from them by working in the household before they leave'.

Other negative coping mechanisms were noted in Al Khayraat, where women IDPs saw an increase in drug addiction among men, leading to an abdication of decision-making responsibilities. Drug use, youth conflict, and theft have increased, according to boys in Bulo Migis.

Families are also skimping on food consumption to help make ends meet. The men in the Bulo Migis FGD said a number of them only eat white rice and drink black tea with no sugar to save on food costs, and ask all household members to consume less and to go and find work. Men will barter or take out loans, while women and girls beg in the streets or from neighbours. A key informant in Bariga Bossaso also said that women have now had to resort to begging, while another in Kalabayr stated that they have had to sell their livestock to survive.

These negative coping mechanisms are also a result of a lack of a support network. The survey shows that $84 \%$ of men and $65 \%$ of women have no neighbours or family members to give them shelter should they need it (Figure 55 ), and $96 \%$ of men and $95 \%$ of women said they have no one to turn to for financial support should they need it (Figure 56).

Figure 55: Whether neighbours or family members can provide shelter if needed

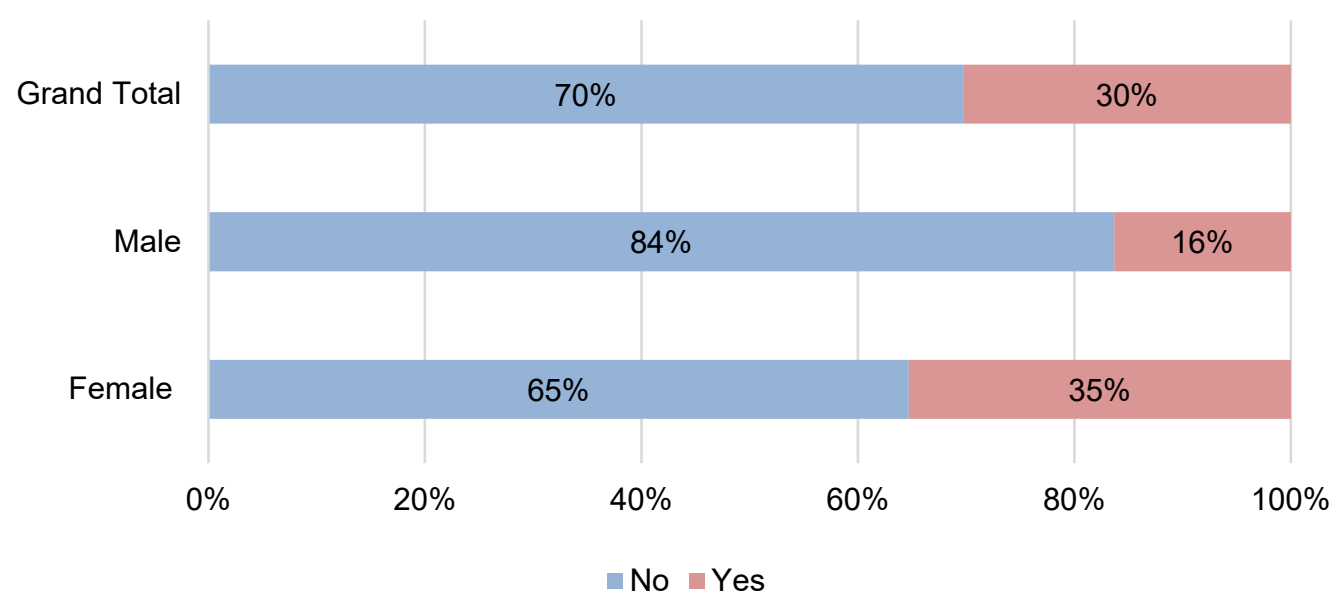


Figure 56: Whether neighbours or family members can provide financial support if needed

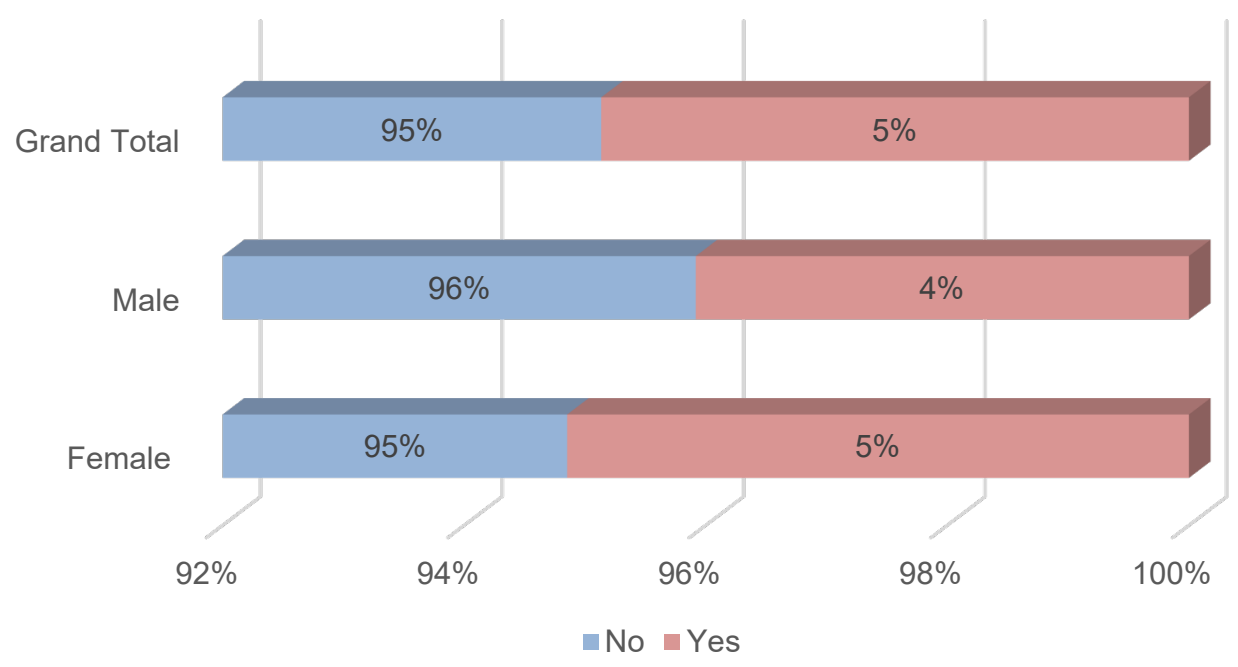

However, there were more opportunities for support through in-kind assistance, with $86 \%$ of men and $67 \%$ of women saying they have neighbours or family that can provide this (Figure 57 ).

Figure 57: Whether neighbours or family members can provide in-kind assistance if needed

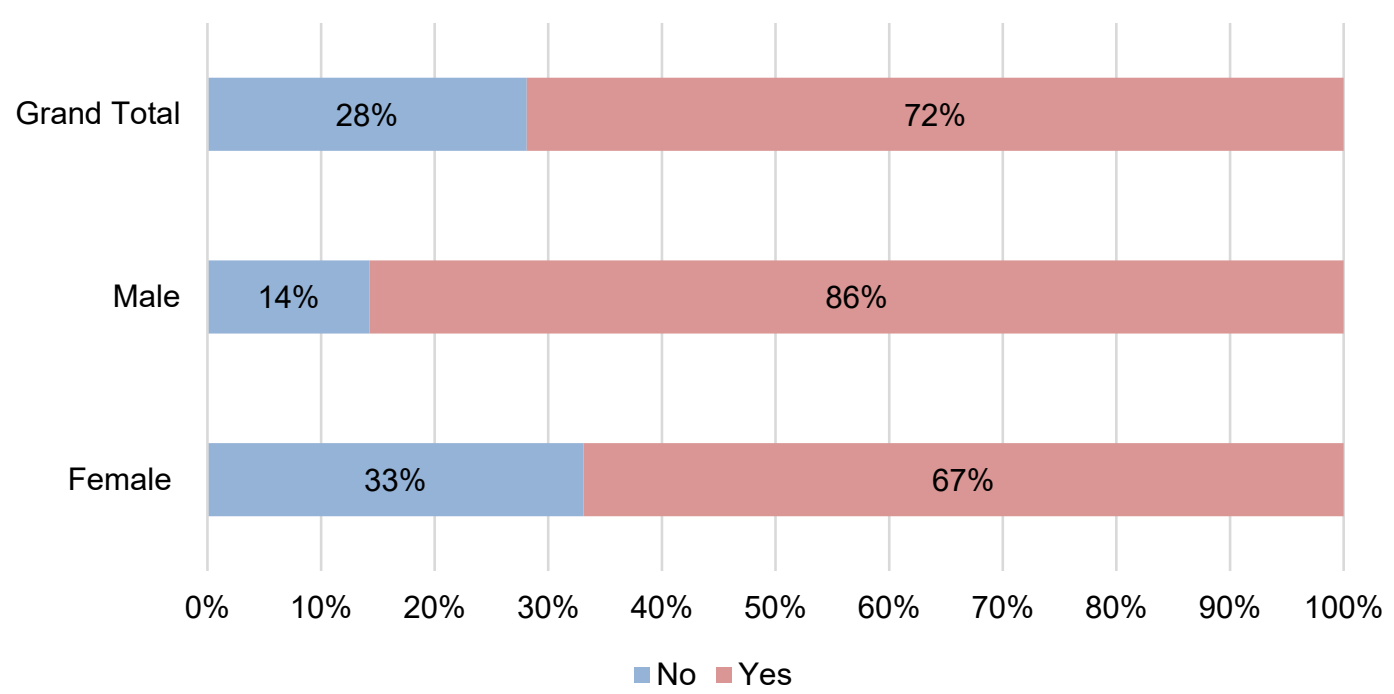

In terms of help and advice, community members turn to their spouse most often, followed by local leaders (Figure 58). More broadly, the community does the same (turns to family support), with the second-most common support being from the NGO sector (Figure 59). 
Figure 58: Who respondents go to for help and advice

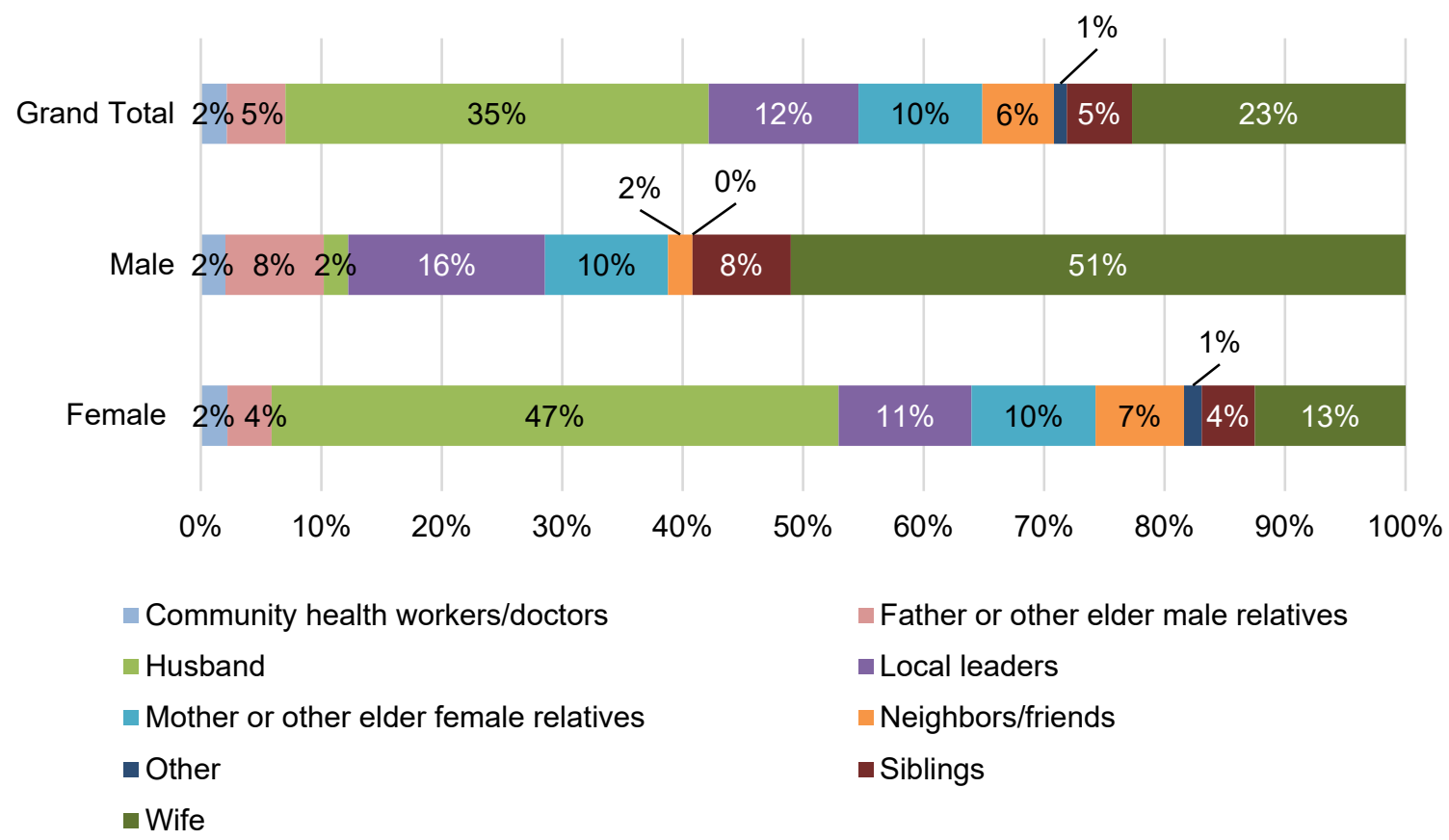

Figure 59: Who the community asks for help

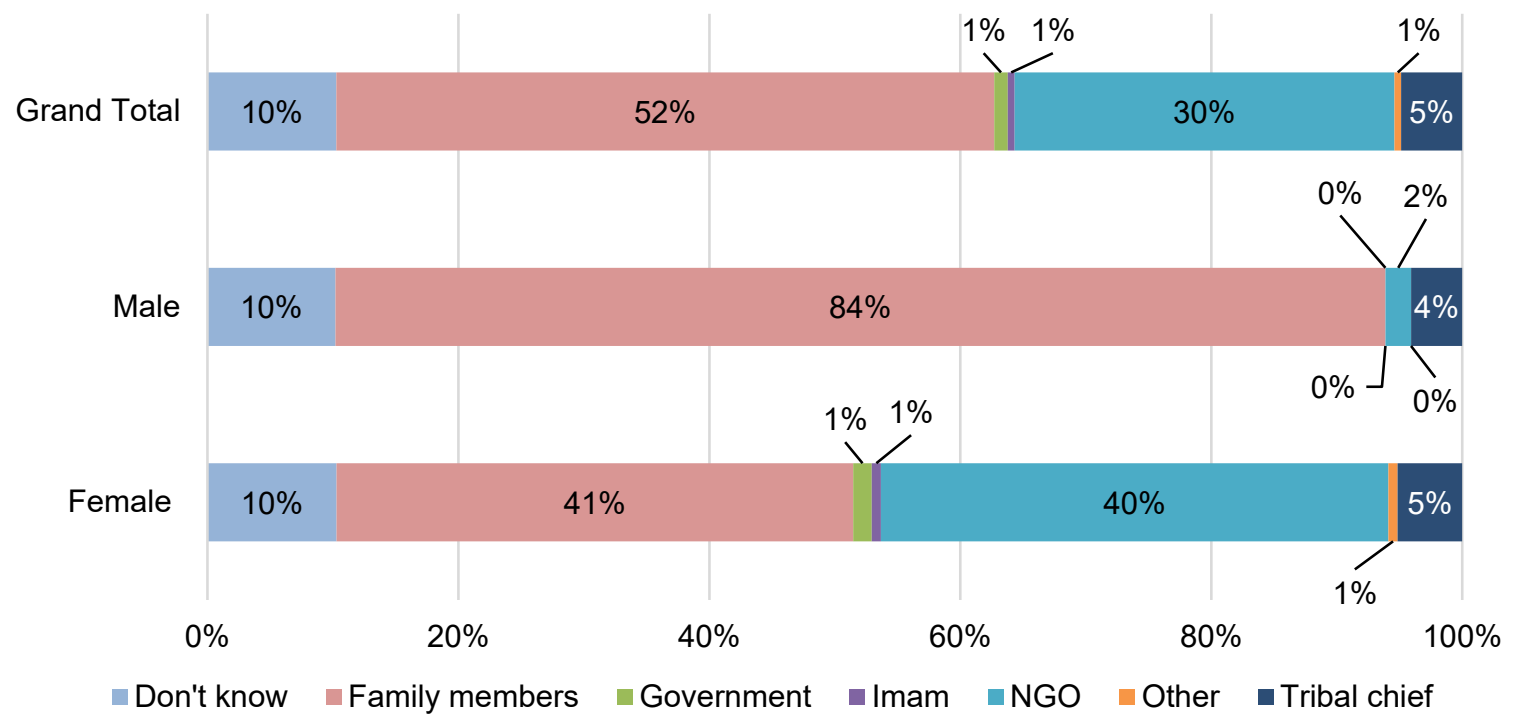

Key informants confirmed that the community comes to them for support regarding livelihood assistance, with one adding that poor families, female-headed households, and families of minority groups ask for support, and the community leadership group contributes and provides support in the form of kaalo or caawimaad. ${ }^{15}$ 


\section{PROTECTION}

\section{THREATS AND RISKS TO WOMEN AND GIRLS}

Safety and security concerns are widespread in precarious environments, with women and girls facing elevated risks of various forms of violence. Routine tasks such as collecting firewood or going to the market leave women and girls open to harassment, assault, and theft, according to two FGD groups and KIls with community members and INGOs. There is a looming fear of rape or attempted sexual assault when undertaking tasks such as using the toilet or working in someone's home. The girls in one village shared that they dread using the toilet at night so instead have something like a bottle to use if they need to relieve themselves. Mothers do not send their daughters to collect firewood or fetch water because they are afraid for them. Girls stopped work activities outside the home because of this insecurity, thus limiting their opportunities.

There is considerable dependence on the men of the family to protect the women and girls. Girls will follow the men when going outside, or tell the boys to fetch things needed outside the home. Serious incidents will be reported to the police, but otherwise no action is taken. One settlement has encountered an increase in drug dealers, alcohol users, armed youth, and thieves. Community organization is needed, with safety and security teams required. Effective lighting is also needed, and even a torch is a pressing necessity.

As a coping mechanism and to mitigate against any attempted violence, women and girls opt to walk in groups, try to complete work before sunset, and even wait for prayer time as this is when the majority of men go to the mosque. They also listen to news updates to keep abreast of what is happening and to be aware of any existing tensions.

The girls in one host community said that they had no specific security concerns but were afraid something could happen to their husbands and fathers. In another location, girls fear hunting animals as this means they are outside the safety net of their home, and are afraid of youth groups as they can create hassle, though it was not clear what type of hassle they were referring to. Other girls' FGDs mentioned that they are fearful of armed groups, especially when they go bathing in open spaces.

Girls typically report any threats or instances of violence to their parents, their mothers in particular, and other close family members. Community members such as traditional elders and religious leaders were identified in one village as those that could provide help.

In one district, men are women's biggest security concern. A constant fear of attempted rape, kidnapping, and theft looms over the women and girls. They go out in groups or with their brothers. Many just stay home or take small weapons with them when outside, as was the case for all the women and girls surveyed. Sticks, stones, and other small items were carried outside the home as an added layer of protection. In certain sites, incidents can be reported to organizations like KAALO or other communitybased groups.

Boys in some FGDs discussed forms of torture, as well as human trafficking and forced and early marriage, as security concerns for girls and women in their area. Women and girls across the FGDs said 
that they were not safe in IDP settings. In two localities, they said that it was hard for a woman or a girl to stay in their household alone, even in the daytime.

Community KIls mentioned several GBV risks (domestic violence, specifically IPV), early marriage, theft, FGM, and rape while fetching water at night - all of which are explored further below.

\section{THREATS AND RISKS TO MEN AND BOYS}

Even though men are considered mostly responsible for security within the IDP camps, this does not prevent them from falling prey to violence as well. Boys sometimes fight while playing football and fear getting injured. Youth in one village face their earned wages being taken by armed groups, which can turn violent, as reported by one young participant. Collaborative actions such as travelling in groups, reporting incidents to police or security officers, and sometimes carrying self-defence tools such as a hammer, were ways boys protect themselves in certain communities. Men seek assistance from the military or police, or report incidents to the chief of the camp.

Some community members face tensions with armed groups, which pose the main threat to men and boys. Boys' protective measures included using hands and legs to defend oneself and leaving communities in the evening if feeling unsafe. A few communities are located on the edges of certain armed groups' activities, which can pose an increased risk.

Men use torches or flashlights, some of which were provided by KAALO as they are important items for families but not on their priority lists. In two sites, IDP men stated that they use sticks as a means of protection. They also indicated that armed gangs, characteristically comprising boys, were responsible for security risks, as well as, in one location, security officers who make false claims and falsely imprison people.

Tensions around armed groups and tribal conflicts were stated as occurring inside the IDP settlements, and in one settlement in particular, even walking inside the camps in the morning can be risky. Another threat is that of fires, whereby fires, either deliberately set or caused by high winds carrying cigarettes or cooking flames, have caused further loss of assets and displacement. Protection issues, as well as unexpected environmental hazards such as floods or drought, can have a significant impact on life and create increased vulnerability.

Local villages fall victim to threats from armed actors and worry about nearby movement. Some communities noted that youth are more susceptible to armed group activity and recruitment. In one village, women remarked that men and boys are susceptible to kidnapping, murder, and threats from armed groups. Community KIls also highlighted thefts and the risk of boys forming gangs, with the risk of conflict and street fights between them. 


\section{GENDER-BASED VIOLENCE}

Many of the focus groups were attuned to GBV, noting increases in violence and various forms of GBV. The IDP women's FGD in Bulo Migis stated that violence has increased in terms of IPV, family problems, sexual assaults, and rapes. Family problems and domestic violence have increased because of the lack of household items to meet basic needs, and men and women losing their daily wages, so lack of money is a stressor. Current levels of unemployment and stressful environments have increased the extent of physical and emotional threats and sexual assault. Before the crises, although there were threats and sexual assault attempts and some cases of rape, normal life was carrying on normally, everyone had tasks to attend to, and they could earn income in order to cover their needs and buy basic household groceries.

In Jowle, participants highlighted incidents of killing women, $\mathrm{FGM} / \mathrm{C}$, and early marriage (although not forced marriage). The Al Khayraat women's FGD identified rape, sexual assault, early marriage, and FGM as forms of GBV that existed in their community. On the flip side, the IDP women in Bariga Bosaaso claimed that there was little rape or sexual assault, but that domestic violence did exist.

All noted that men and teenage boys perpetrate the violence, and in Jowle bus drivers were also identified as culprits. Due to rising sexual assault cases in the community and town, the women's IDP FGDs stated that they could not allow their girls to work, even in other houses providing domestic labour or laundry services.

In Carta, the host community women acknowledged the link between domestic violence and economic stress, resulting in girls marrying early (sometimes by Qudbo Siro ${ }^{16}$ ) to reduce financial burdens. KIls from across the communities added that rape, FGM/C, and early and forced marriage are the most common types of GBV in their communities.

\subsection{VIOLENCE AGAINST WOMEN}

Violence against women was seen to more prevalent now than before. Domestic violence, specifically IPV, has increased, with cases of husbands forcefully taking earnings from their wives mentioned by the girls' FGDs in Bariga Bosaaso and Jowle. The girls' FGD from Bulo Migis reiterated that violence has increased in town and in the IDPs, and in Budunbuto girls reported that incidents increased during droughts and periods of disease, with domestic violence causing family break ups.

Both the women and girls saw the idea of safety in numbers as a critical mechanism to protect themselves from this type of violence, so were traveling in groups when outside the home. The girls also cited traveling with family members or brothers when running errands. Some mentioned having mobile phones as a deterrent to being attacked, so that they can call their relatives immediately or use the phone as bait for the thief rather than having anything else stolen or being raped.

The boys' and men's FGDs linked the increase in domestic violence to financial issues and lack of income, and the men in Bulo Migis noted that the economic crisis has brought increased tension in families, so every household member now has an economic responsibility. A key observation from the boys' FGD in Bariga Bosaaso was that the increased authority exercised by women as they are getting more work than men (who used to earn more before the COVID-19 measures) is creating a shift in family dynamics and causing conflict between spouses - highlighting ingrained social norms around gendered 
roles and responsibilities. All KIls across the communities repeatedly mentioned the increase in domestic violence, specifically IPV, as a result of financial stressors, with one key informant saying that this was happening everywhere. One INGO key informant added that:

'natural and man-made disasters have increased the difficulties of finding out basic life needs and this has disproportionately affected women and girls, including those with disabilities. Due to displacement, social structures have been broken and women and girls' support systems disrupted, exacerbating [their] vulnerability to shocks and [the] risk of violence both at home and in the community. Women have increasingly become heads of households, and [the] main breadwinners for their families, and gender roles have shifted, putting women in a position where they have increased burden as they play double gender roles. This has put them at more risk as they often travel far from the shelters in search of work, food and water. For male-headed households, the risks of IPV directed at women has been heightened as women are expected to do more than their traditional gender roles, including contributing to family income. The diminished incomes have resulted [in increased] tensions in the household and women find themselves at risk of IPV.'

One key concern is that specific services for women in the areas included in this study are limited, with the survey showing that $37 \%$ of the women indicated that they do not have access to women's services (Figure 60 ), due to lack of money to pay for services (mentioned by $50 \%$ of female respondents), or a lack of health facilities altogether (31\%) (Figure 61). Community key informants (both men and women), especially those that are asked to intervene in such matters, added that there is no formal system but that the community calls on them for support (according to one male and two female key informants), or to resolve the issues in a traditional court (two male key informants). The key informants said they provide counselling and moral support on GBV. Only three community leaders (in Bulo Migis, Bariga Bossaso, and Al Khayraat) added that NGOs provide support, and one key informant mentioned medical and other referral mechanisms. In relation to access to medical care, limited medical facilities were noted by the IDPs, and those that do exist have reduced resources, including only a small supply of medicine such as painkillers, and no other critical prescriptions available. Most of the villages have no medical facilities in place, and those that do again only have painkillers available as medication.

However, when asked about information on GBV response services specifically, several key informant respondents said they were aware of these and mentioned NGOs as providing support, with half knowing about the existence of a hotline. One key informant added that the community still does not know this information - showing that if there is some knowledge, that knowledge stays with the community leaders. 
Figure 60: Access to sexual and reproductive health services by women

Women only - access to sexual and reproductive health services

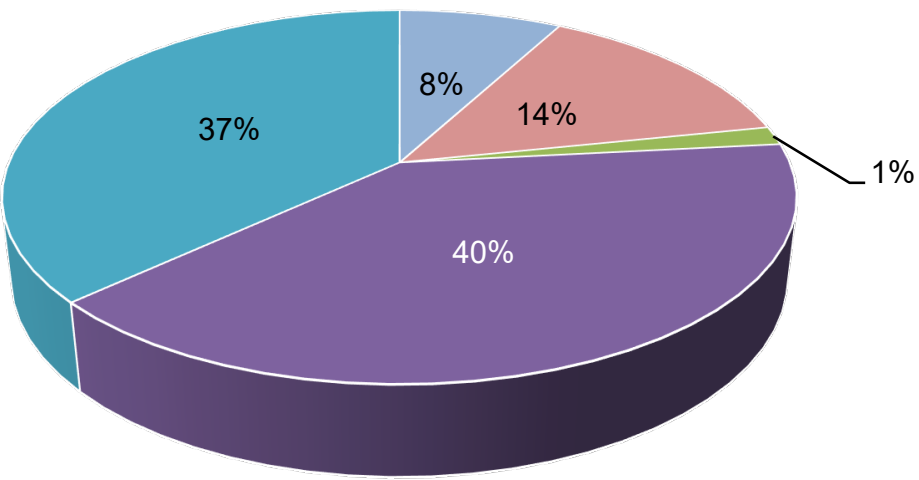

$$
\begin{aligned}
& \text { - Attended delivery } \quad \text { - Clinic/laboratory } \quad \text { - Family planning } \\
& \text { - Maternal health } \quad-\text { None of the services }
\end{aligned}
$$

Figure 61: Reasons why women do not have access to services

If none, why do you not have access to women's services?

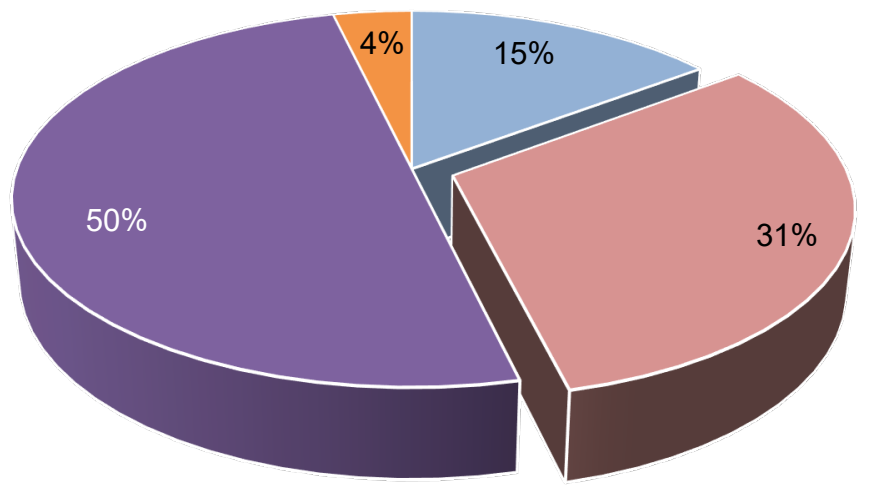

- No female health staff

-No health facilities

- No information

- No money to pay for services

- Other

\subsection{CHILD MARRIAGE}

In the focus groups with the girls, boys, and women IDPs, child marriage was identified by some as a GBV risk, while others stated that it was a traditional norm. Some groups alluded to the choice sitting with the child, but the majority confirmed that ultimately the parents decided, more specifically the fathers. This was confirmed by the survey data (Figure 11). 
The Bariga Bosaaso girls' FGD commented that in that IDP camp it is normal and traditional for girls to marry when they are children, and both parents make the decision to marry girls or boys at an early age. The reasons for marrying girls at an early age are fear of pregnancy out of wedlock, and fear of sexual harassment or assault. If a girl is married, it is believed that is will be less likely that she will be victimized. The girls mentioned wanting relief from the heavy workload in the household and that being married might give them the ability to manage their life and time. The girls in Bariga Bosaaso also commented that having sex without being married or when in an illegal form of marriage (Qudbo Siro) is quite common. Key informants confirmed that Qudbo Siro is quite common In Jowle. The girls agreed that there is child marriage and that the decision is made jointly by the parents, but the ultimate decision is made by the father. The boys in Jowle mentioned masaafaysi, a practice whereby a girl travels to a remote area and gets married without her parents' consent. The situation in Al Khayraat is similar, where the girls stated that there is early marriage because of fathers preferring to marry their daughters off while they receive small cash sums.

The boys' FGDs in Kalabayr and Carta remarked that parents do not select spouses for them, but they do select the person they are interested in marrying their child to, based on mutual agreement and acceptance of the fiancés. This statement leaves it unclear as to whether the child participates at all in choosing a spouse. Contributors in the boys' FGD in Bulo Migis observed that child marriage is increasing and that fathers take the decision alone, based on the family's needs. When boys are to marry, they consult both parents. The Kalabayr boys' FGD said that as soon as a person turns 14 , he or she can get married, so child marriage is not really a term used in the community. If a boy decides to marry, they make their own decision whether it is their choice to get married or not. The Carta boys' FGDs stated that people marry when they are teens, at around 13-14 years old, especially girls. In the current crises, however, child marriage has increased for girls only. Boys cannot handle the family responsibilities after marriage during the current harsh times. This contradicts what the host community women in Carta stated: that there are no child marriages in the community, and that if children wanted to marry the parents decided based on their child's choice. They also specified that girls marry early and sometimes they marry men in Qudbo Siro. Like the Carta boys' FGD, the boys in Bariga Bosaaso have also seen an increase in girls getting married. The fathers make the final decision, but youth tend to consult their mothers on this matter.

The survey results confirm that more girls are married at a younger age than boys: $8 \%$ of both male and female respondents answered that boys get married before the age of 18 (Figure 62), compared with $40 \%$ of men and $44 \%$ of women who said that girls get married before the age of 18 (Figure 63 ). 
Figure 62: Current age of marriage for boys

At what age do boys usually get married now?

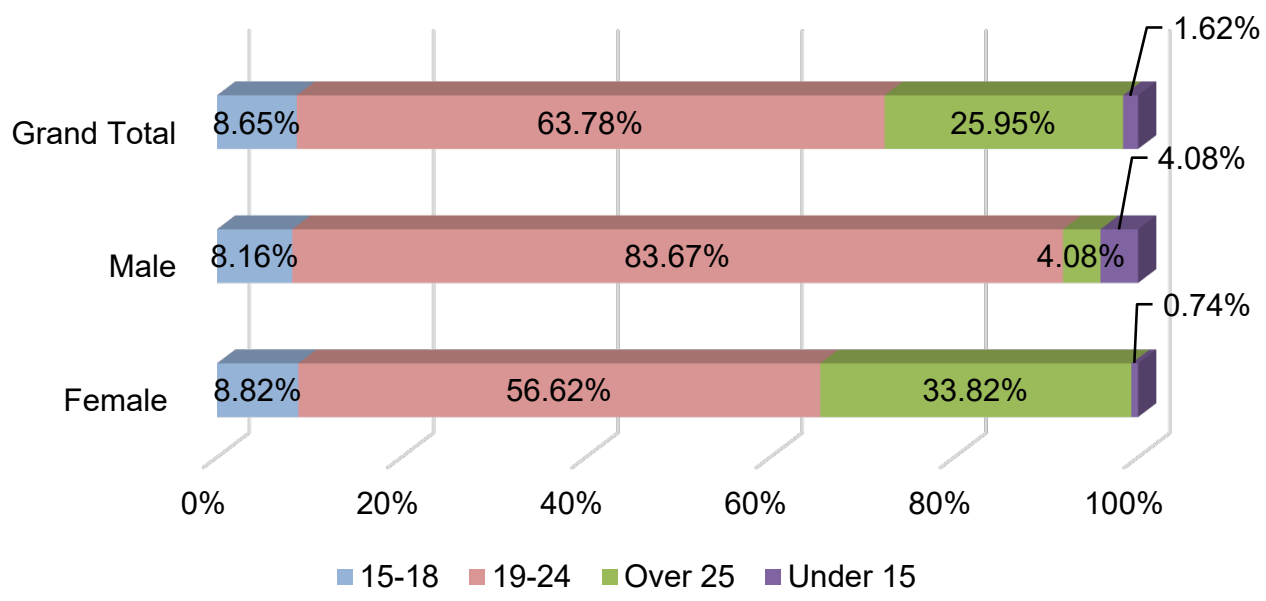

Figure 63: Current age of marriage for girls

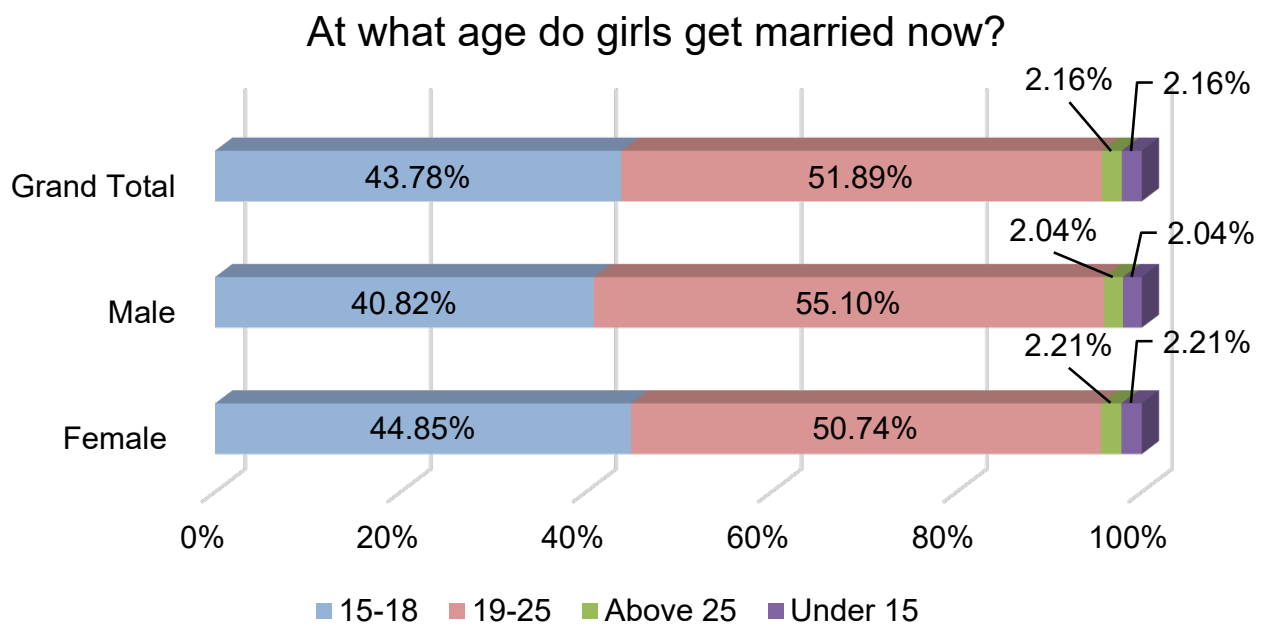

Key informants across all the communities agreed that early marriage is increasing, and saw this as a risk only for girls, with no specific mention of boy's early marriage. On the prevention of GBV, all KIls with community members suggested that awareness raising was needed, along with lighting at toilets and homes across all locations, and closer WASH facilities in Budunbuto. Two male key informants mentioned the need to comply with Sharia law. Notably, one male KII mentioned that men should do the WASHrelated housework to prevent women from being exposed to risks. 


\section{ACCESS TO INFORMATION}

Effective and reliable communications and information sharing in times of crisis are critical and lifesaving, especially for the most vulnerable populations. For the IDP communities, radio and hotlines seem to be the main means of sharing information. Women IDPs in Jowle noted they receive information from $\mathrm{MCH}$, the radio and the Ministry of Health, and women in Al Khayraat also mentioned hotlines. Also, in Al Khayraat, community women and girls have access to information and prevention material around the coronavirus pandemic, in addition to two trained focal points provided by organizations such as KAALO. The Ministry of Health has established a hotline to provide updated information to communities, and there is a woman in both Bariga Bosaaso and Saacada who works at the Ministry of Health and keeps the community abreast of the situation. In Jowle, social media is another form of information exchange to obtain news. All women IDPs in the FGDs stated that they receive information about coronavirus through in and outgoing calls. Some also mentioned radio and television as information channels.

Women in the host community of Kalabayr noted calls from the biggest telecommunications company in Puntland and awareness outlets provided by $\mathrm{MCH}$, while those in Carta, Cuun, and Kubo use radios, phone calls and, if available, the television to receive information, while in Budunbuto hotlines are used. Host women in Geri Hel said they do not get the same information as men and boys because they stay at home.

When asked if information is tailored to the needs of pregnant and breastfeeding women, or people living with HIV, pre-existing conditions, and disabilities, and older persons, the women's group in Bulo Migis said they do not have people living with HIV and pre-existing conditions and the rest are able to receive information from the hotline, $\mathrm{MCH}$, or their family members. The Bariga Bosaaso FGD also stated they did not have people living with HIV and pre-existing conditions in their community, but noted that some women have taken an activist role in terms of the rollout of information. In Jowle, pregnant and breastfeeding women and people living with HIV receive information from $\mathrm{MCH}$. In Bulo Migis, Bariga Bosaaso, and Al Khayraat, the FGDs noted the need for messaging to vulnerable groups such as pregnant and lactating women, people with chronic illness, and elderly people, so they can stay up to date with important information.

The lack of information tailored to the needs of pregnant and breastfeeding women, people living with HIV or pre-existing conditions, elderly people, and people with disabilities is a huge gap. The need for accurate information from qualified healthcare professionals in the local language is crucial.

\subsection{ACCESS TO HUMANITARIAN SERVICES AND INFORMATION}

In regard to access to humanitarian support, many of those consulted were unaware of ways in which to engage with humanitarian organizations. The girls' FGDs stated there is no access to humanitarian assistance and they do not know where or with whom to express any concerns they may have. Assistance is limited and they are not personally consulted about their needs. For the most part, the majority were unaware of a hotline number or place to provide feedback or complaints. In Al Khayraat, the girls got information through calls, media, television, and public spaces, and some respondents said they know of some outlets and do provide feedback to KAALO. 
For the boys, they have never been asked for a meeting or consultation regarding humanitarian assistance in Bulo Migis and Bariga Bosaaso, nor are they aware of where to access relevant information. However, in Kalabayr and Carta, it was noted that there is access through various media channels and mobile awareness actions. Across all the boys' FGDs, none knew how to provide feedback or submit a complaint about an NGO worker or a humanitarian organization.

Across the six host community men's KIls, none receive information from humanitarian organizations and there is no knowledge of feedback loops, except for in Budunbuto, where KAALO provides relevant information.

This lack of community engagement, consultation, and access to information was further confirmed by the survey: $90 \%$ of men and $77 \%$ of women said they have not been consulted on their needs by a humanitarian organization (Figure 64).

Figure 64: Whether respondents have been consulted by aid organizations

Have you been personally consulted about your needs by aid organizations?

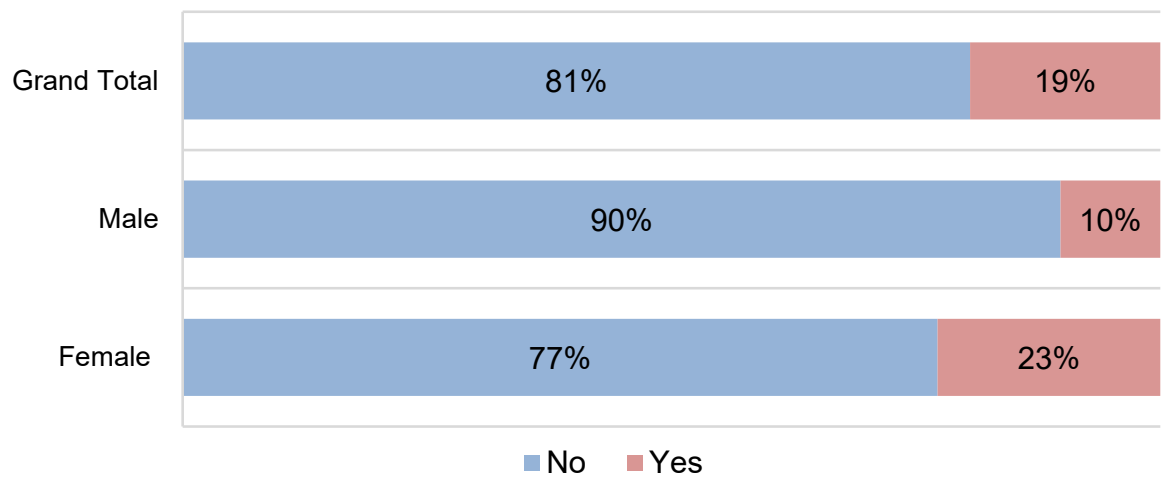

When asked about their knowledge of complaint mechanisms, $92 \%$ of men and $65 \%$ of women said they did not know how to put in a complaint (Figure 65). In relation to feedback, $94 \%$ of men and $76 \%$ of women had never provided feedback to an organization (Figure 66). On a more positive note, of those that did provide feedback, all the men and $97 \%$ of the women said their feedback was taken into account (Figure 67). 
Figure 65: Whether respondents knew how to complain to aid organizations

Do you know how to put in a complaint about misbehaviour by an aid worker?

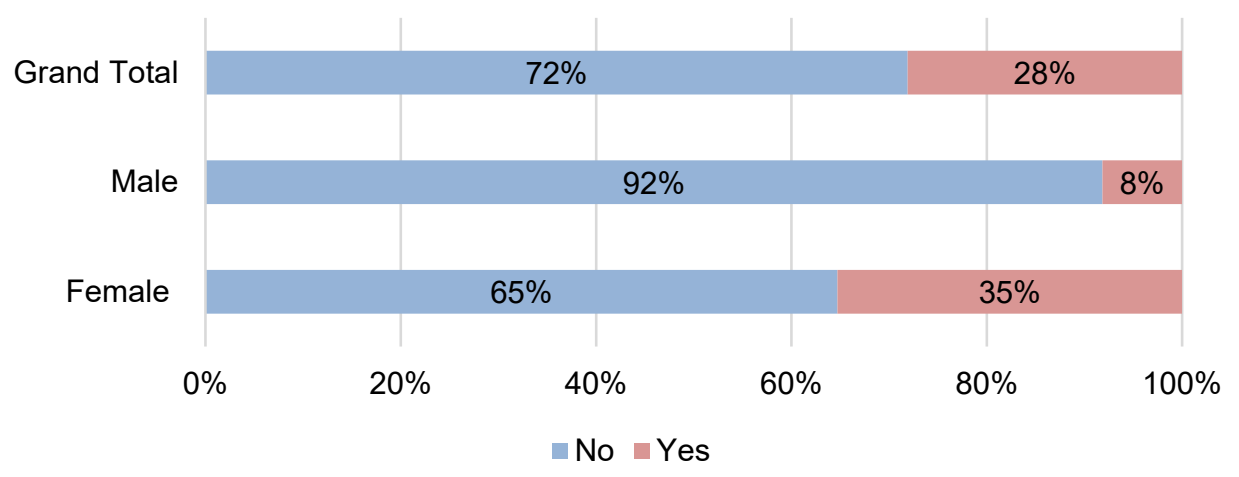

Figure 66: Whether respondents had ever provided feedback to an aid organization

Have you ever provided feedback to an aid organization?

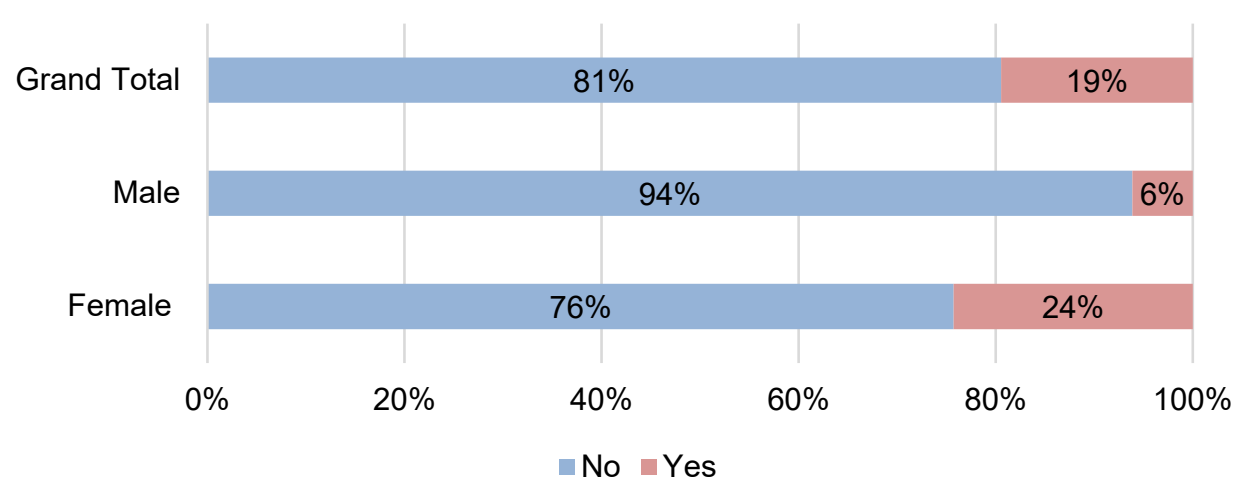

Figure 67: Whether respondents felt that their feedback had been taken into account

If yes, do you feel your feedback was taken into account?

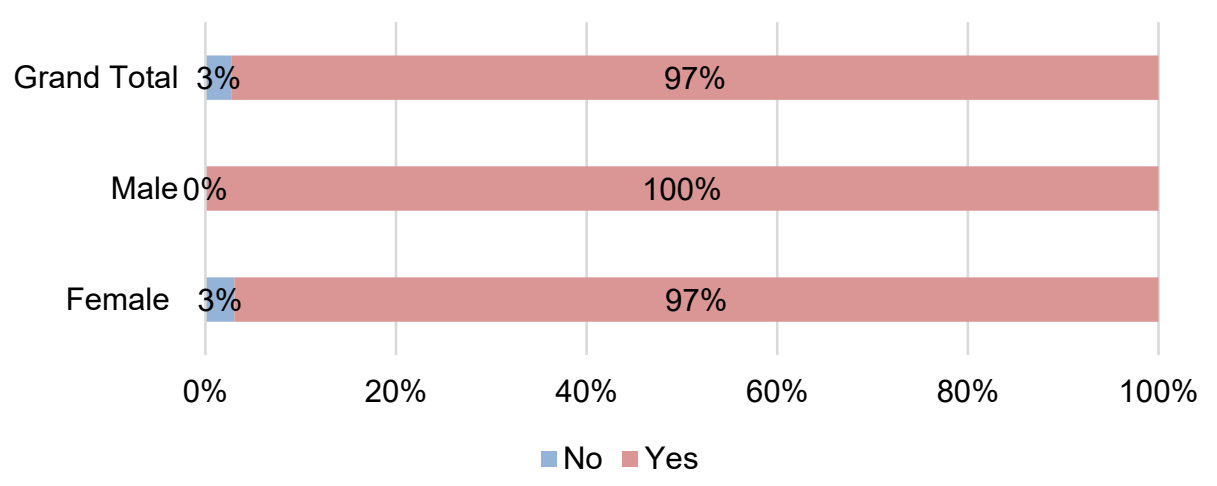


On information about accessing humanitarian services, $96 \%$ of men and $85 \%$ of women said they do not have the information they need to access assistance (Figure 68), again showing the need for much more community engagement.

Figure 68: Knowledge of how to access humanitarian assistance

\section{Do you have the information you need to access humanitarian assistance?}

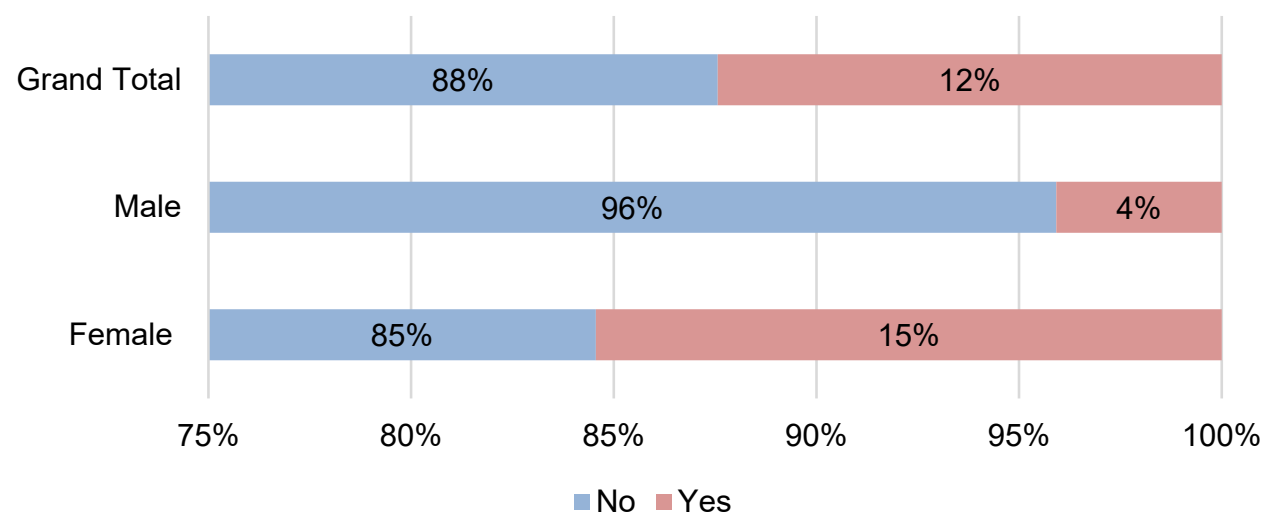

Key informants across the communities seem to have knowledge about the humanitarian services available, but there was no indication that this knowledge gets passed to the community members, especially to specific vulnerable groups.

\section{COVID-19}

The public health and security threat of the coronavirus pandemic is far reaching, and its gendered impacts are readily apparent. Women, girls, and other marginalized groups such as IDP communities are facing decimated livelihoods and gross economic vulnerability, restrictions on mobility, reduced or eradicated access to and control over vital resources, a greater burden of (unpaid) care work, increased risk of GBV/IPV, and limits on access to justice and legal systems. According to the Somalia Ministry of Health, as of 16 March 2021 there had been 9,328 confirmed cases of coronavirus, and there were 4,679 active cases and 379 confirmed deaths. ${ }^{17}$ No specific data were available for Puntland State.

The women IDPs in Bulo Migis noted there no health services related to COVID-19 are provided to the community outside of awareness raising regarding the use of masks, gloves, proper handwashing techniques, and social distancing. However, in this IDP community, these protocols cannot be implemented due to the living conditions, such as large numbers of family members living within one shelter or room. In Bulo Migis, Bariga Bosaaso, and Al Khayraat, according to women IDPs, if someone is suspected of having coronavirus, they are treated with cultural remedies, such as black seeds, ginger, and lemon with warm water and honey. The community in Jowle, however, stated that services can be accessed there in the $\mathrm{MCH}$. 
The host women in Carta received coronavirus information through the radio, Golis ${ }^{18}$ calls, and media awareness campaigns. There was no messaging specifically tailored to people living with HIV, disabilities, or pre-existing conditions.

The survey results indicate that awareness raising on COVID-19 had been widespread, with all men and $99 \%$ of the women saying they had heard of the virus (Figure 69), and most having heard of it through the radio (Figure 70). It is noteworthy though that the prevention measures are not as well known by women, with $45 \%$ of the women not knowing about them, but only $12 \%$ of men (Figure 71 ).

\section{Figure 69: Knowledge about coronavirus}

\section{Have you heard of coronavirus?}

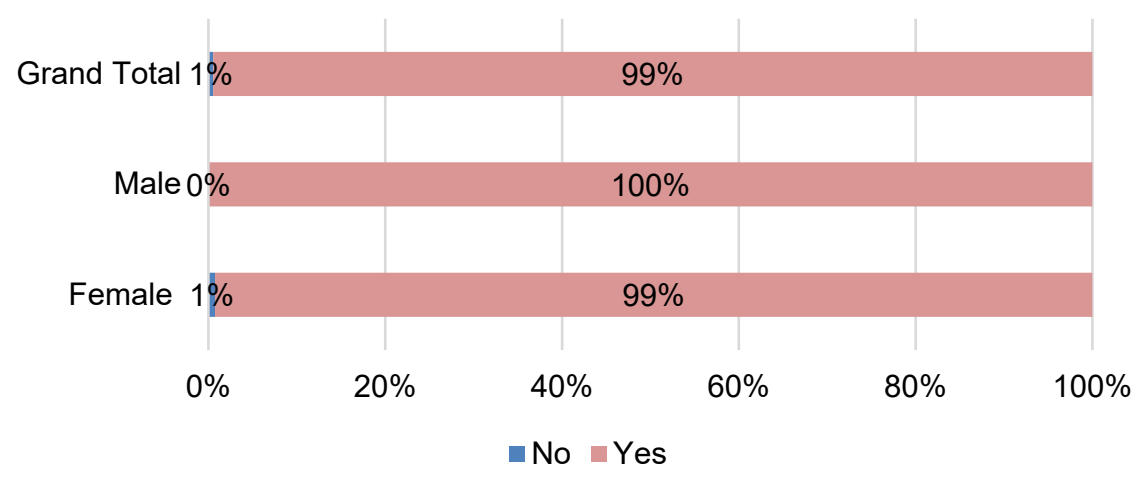

Figure 70: Sources of information about coronavirus

What are your sources of information about coronavirus?

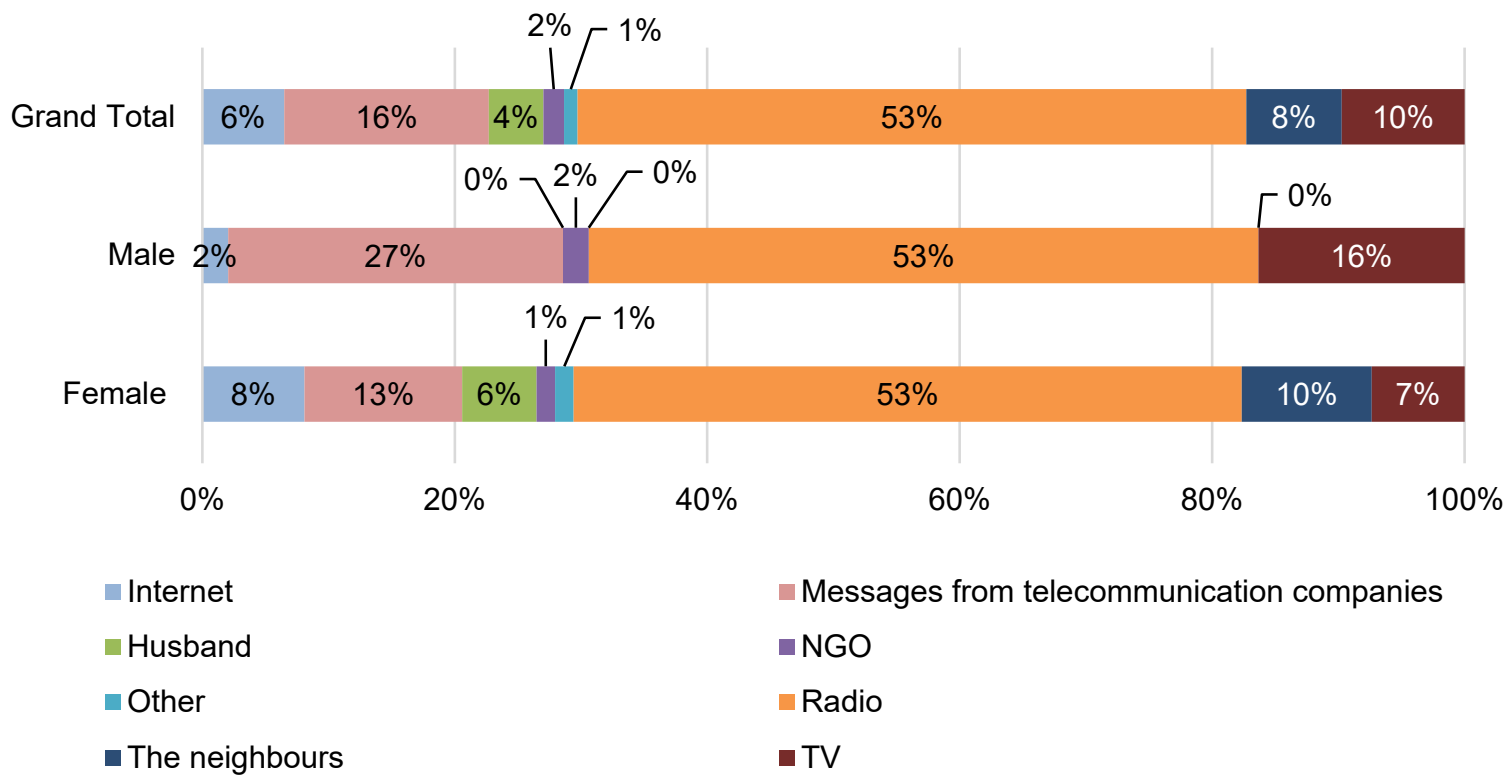


Figure 71: Knowledge of how to protect against coronavirus

Do you know the prevention measures against coronavirus?

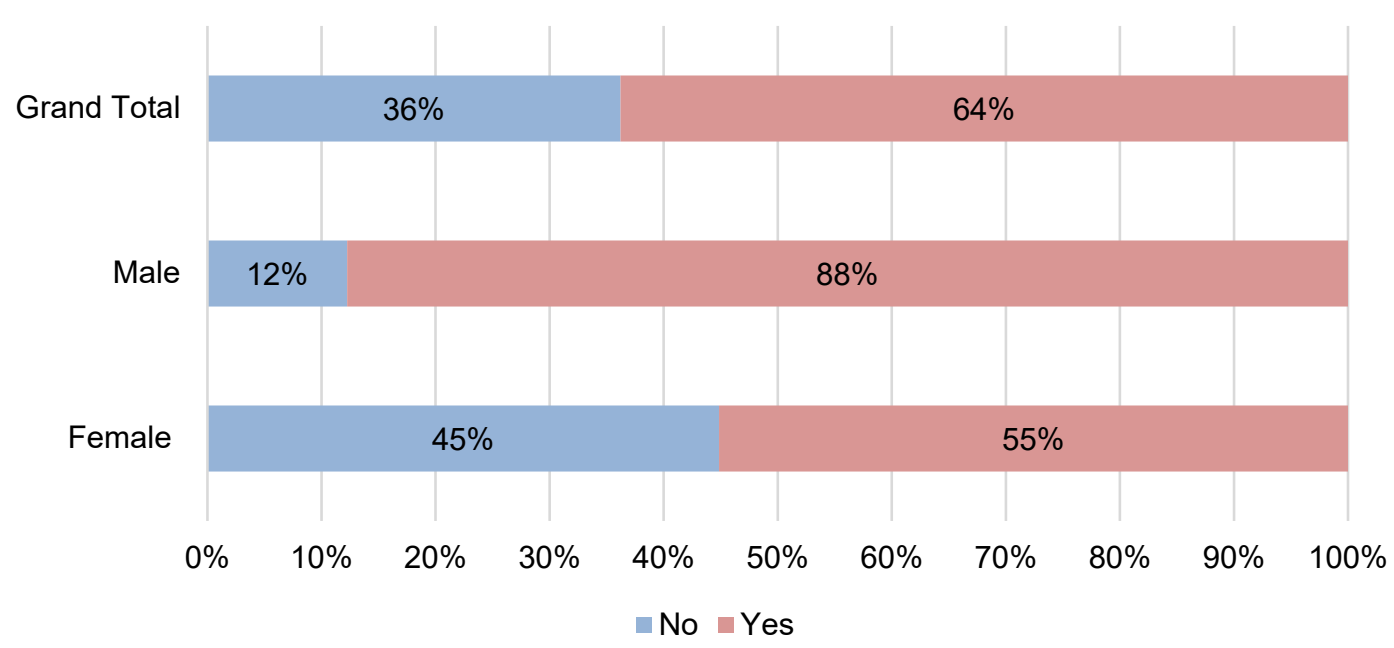

Furthermore, all community KIIs mentioned the impact of the crisis on livelihoods, and when asked whether community members who have COVID-19 faced stigma, two community key informants said they did, while the others did not. The majority said that women would not be able to quarantine if they have COVID-19 because of family and work duties, and also due to the fact that most communities do not have health services. The KIls did not ask whether men would be in the same situation.

\section{CAPACITIES OF DUTY BEARERS}

Out of the nine KIls with representatives of INGOs and government structures, three INGOs had both the capacity and the resources to ensure gender mainstreaming in their projects, as well as specific responses for GBV survivors. Two of the duty bearers are best placed to provide support on the protection and GBV risks identified in this analysis, and coordination should be enhanced on those issues to ensure a rapid response.

The capacity of the INGOs and government bodies regarding WASH varies, but it is clear from the community findings that more needs to be done to ensure that the needs of women, men, boys, and girls are safely and adequately met. Consultations and engagement with the communities is not systematic.

Capacities are similarly varied in relation to EFSVL, with one INGO that is acutely aware of the needs and risks that different groups are encountering. All mentioned food security as a key issue which they respond to with either training or capacity building, but suggested that financial grants and more sustainable approaches are needed, especially given the compounded effects of the recent crises on food security and livelihoods. There seems to be a focus among all duty bearers on women as beneficiaries, but this does not take into consideration the gender roles that are present in the communities, for example the need to look at care work and share responsibilities at the household level. 
On protection and GBV, all the duty bearers highlighted the distinct needs and vulnerabilities of the IDP communities, as well as of women and girls in particular. One INGO key informant mentioned that IDPs face unique risks of GBV and sexual exploitation due to the limited security in the IDP settlements, poor living conditions, and limited clan protection, and that the attendant separation of many women and girls from community and familial support structures, as well as from traditional livelihood activities, also contributes to an increased reliance, particularly by women, on marginal, inconsistent, and hazardous livelihood strategies, which often increases exposure to violence. Another INGO key informant mentioned that 'women and girls continue to be most vulnerable communities in this area. Girls endure GBV forms including FGM, [and] child, early and forced marriage'.

An INGO key informant further mentioned that a 'shortage of livelihoods and income-generation activities remain in the IDP and in the host community families, and weak shelter poses further risks to women and girls'. The negative community protection mechanisms, lack of police presence, inadequate lighting, and an increase in the number of female-only households have turned IDP settlements into fertile ground for GBV. The incidence of GBV is alarmingly high, particularly among IDPs, in the form of rape, physical assault, IPV, and sexual exploitation and abuse, and occurs in situations where there is a lack of access to formal justice mechanisms and extreme marginalization of women under traditional justice structures.

Several INGOs and government bodies had conducted awareness raising sessions in the communities and training for staff on these issues. A local NGO has further implemented a project focused on social norm changes, with the engagement of religious leaders as role models. These types of projects should be built on and expanded. The existing referral pathways and links between the organizations that provide services and the community need to be strengthened. The women's forums also established by the same local NGO need to be built on and expanded in order to ensure women's empowerment and leadership, together with the management of any potential GBV risks this could create.

Further improvements, as mentioned by the duty bearers, include the need to establish comprehensive opportunities for a capacity development plan for gender integration and delivering tailored refresher courses on gender considerations for staff. In particular, there is a lack of technical experts and participatory learning activities for partners to promote staff capacity. There is limited gender-sensitive awareness in the region to facilitate and reflect understanding and practice gender considerations, and little coordination in working with stakeholders and local partners to understand the local context (norms, cultures, laws, customs) and capacity (knowledge, skills, attitudes) towards gender integration. Finally, there is a lack of capacity around advocacy and information management systems, limited financial resources, and ongoing security concerns. 


\section{RECOMMENDATIONS}

Below is a non-exhaustive list of recommendations based on the findings from the focus group discussions, survey, and key informant interviews. These include observations from government and NGO interviewees of potential areas of enhancement in Puntland's responses to the current crises.

\section{Recommendations for duty bearers}

- Ensure gender-sensitive programming across all sectors and engage with local communities, especially women and girls, for the consultation and creation of projects before they are implemented; use the findings of this analysis to make changes and improve current projects.

- Following direct requests from community members, consider the implementation of educational centres and sensitization on education for girls, but also specific youth projects aimed at boys.

- In line with respondents' most-requested items, ensure distributions of blankets, firewood, and clothing (for both men and women).

- Ensure gender-balanced teams with the ability to listen to women and men, and girls and boys.

\section{Specific recommendations for Oxfam sectors}

\section{Gender}

- Build on existing women's groups in all locations, with training and financial support as well as with awareness raising in the community on the benefits of the women's groups and the importance of investing in girls' education. In IDP settings, work to sensitize communities on women's participation in community decision making and support women to do so.

- On female leadership within mixed community leadership, build on the existing women leaders - such as peace promoters, women doing awareness raising and women fundraising for causes such as a mutual aid fund to help others in the community.

- Provide specific support for women leaders, together with male role models that support women leaders to challenge gender stereotypes at the community level.

- Ensure mixed community meetings are facilitated by both male and female staff.

- Engage community boys and men, as well as religious and cultural leaders, in a transformative male engagement approach.

- Start projects that focus on alleviating the burden of household care workloads, and on gender norm changes at both household and community levels to ensure a gender-equal split of household responsibilities and decision-making responsibilities.

- Ensure delivery of basic gender training for government, local partners, and community leadership.

- Consider learning sessions and reflections with field teams, community leadership, and community groups (men and women separately) on topics such as women's participation and overcoming barriers to it. 


\section{Access to information and community engagement}

- Ensure radio distribution across the locations as this is the preferred means of information sharing.

- Continue conducting community consultations with men and women separately to identify their preferred channels of information and consultation.

- Target specific vulnerable groups such as pregnant and lactating women, people with chronic illness, and elderly people though more analysis as to their exact needs and preferred ways to access information and community engagement.

- Engage with women and girls continuously throughout projects and continue planning through engagement with women, men, boys, and girls and their suggestions to improve programming.

\section{Safe programming and safeguarding}

- Given the vulnerability of women and girls highlighted in this report, all Oxfam safeguarding and safe programming steps must be taken before any intervention to ensure that all programme beneficiaries have equitable and safe access to the projects.

- Conduct thorough risk analysis and continuously monitor data to identify any potentially harmful impact and adapt the response accordingly.

- Work with protection teams and safeguarding focal points to establish confidential referral information within the project teams and within the communities.

- Ensure that only female staff work with women's groups, and male staff with men groups.

\section{WASH}

- Urgent WASH measures should be taken to provide an immediate solution to the inability to use the toilet at night (consider pots or other hygienic solutions instead of the existing plastic bottle solution), as well as measures to ensure clean water.

- Construct and install sufficient safe and sex-disaggregated WASH facilities and consult women and girls on water point, latrine, and bathing locations to ensure safety and privacy. At a minimum, ensure locks and lighting (either by distributing lanterns or by providing lighting around the entire area, not just the toilets).

- Ensure sufficient and regular soap distribution across communities.

- Ensure gender-sensitive dignity kits and hygiene items are provided in all project locations and, following more in-depth consultation with women and girls on menstrual hygiene management, ensure they are provided in sufficient quantity and regularly. If they are reusable, ensure access to soap and safe water for hygiene maintenance.

- Consult women and girls, and men and boys, on ways to reduce and redistribute the care work of women and girls around WASH.

- Conduct sensitization sessions by women staff for women and girls on menstrual hygiene management beliefs, and the cleaning and disposal of menstrual items.

\section{EFSVL}

- To meet the huge need, consider cash assistance across locations if possible and conduct a risk analysis to consider all possible risks to such distributions (safety around where to keep the money, safety for female heads of households, increased risks of IPV, etc.).

- Ensure programmes tackle women's care load versus decision making around EFSVL, so that care work is reduced and redistributed among spouses and decision making is shared. The few role- 
sharing examples should be used as positive models across communities and sensitization around this included in any EFSVL project.

- Consider educational and vocational skills training and formal education for young men and women, with a specific youth programme.

- Consider the creation of women's savings groups, but with similar support to men and with sensitization around role change at the household level to minimize IPV.

- Build on current women's groups and provide capacity building and training on empowerment.

- Consider further business and financial literacy programmes.

\section{Protection and GBV}

- Respond promptly to any protection risk or violation of the rights of women and children by establishing efficient referral systems with locally available service providers and facilitate inter/intraorganizational referral of cases.

- Establish positive and constructive relations with local communities, IDPs, and other relevant stakeholders to ensure the protection of vulnerable groups.

- Coordinate with CARE and IRC around the case management of GBV survivors.

- Assist GBV survivors with psychosocial first aid services.

- Contribute to the organization and implementation of awareness campaigns on GBV.

- Assist in the training of communities, partners, and other stakeholders to further capacitate and strengthen their understanding of international protection principles and practice.

- Conduct a quarterly protection, gender, and accountability joint monitoring mission to the areas where the humanitarian programme is running WASH and EFSVL projects.

- In coordination with protection actors, find solutions and community protection methods to the risks from drug use, youth conflicts, and theft.

- Provide support with GBV sensitization, specifically around IPV and early marriage sensitization.

- Community-based protection programming is needed across locations.

- Community cohesion programmes are needed to reduce tensions around armed groups and tribal conflicts in the IDP settlements.

- Address the most common forms of violence experienced by adolescent girls at the inter-personal, family, and community levels, engaging the support of parents and caregivers and establishing community accountability for the safety of girls.

- Engage on information sharing and creating support networks for families and communities around early and/or forced marriage, including the practice of Qudbo Siro.

- Provide economic support initiatives or incentives to families to help alleviate financial strains that may lead to child labour or early marriage.

- Prepare all project teams with referral information for instances when feedback included references to GBV or other protection-related concerns that required a specialized response. 


\section{Monitoring, Evaluation, Accountability and Learning}

- Ensure gender-sensitive and anonymous feedback and complaint mechanisms are available, so that women and men feel confident when filing their complaints or feedback.

- Establish gender-specific platforms for in-person feedback, allowing male and female beneficiaries to feel comfortable discussing sensitive information in the assessments, and in implementation conducting separate and age-appropriate FGDs for collecting feedback and recommendations. 


\section{NOTES}

1 Action In Semi-Arid Lands (asalsom.org)

2 Somaliland shilling - Wikipedia. Information on the currency exchange can be found here: https://en.wikipedia.org/wiki/Somaliland shilling. However, the values mentioned are from our staff using the street value.

3 BBC. (2019). Puntland Profile. Retrieved 16 March 2021, from https://www.bbc.com/news/world-africa14114727

4 Inter agencies, UN OCHA and government droughts assessment reports December 2016.

5 Retrieved from 2-9-map478. gif $(330 \times 355)$ (osu.edu) on 8 April 2021.

6 Earth Observatory. (2020). Gati Makes Historic Landfall in Somalia. Retrieved 16 March 2021, from https://earthobservatory.nasa.gov/images/147576/gati-makes-historic-landfall-in-somalia

7 UNSOM. (2019, 9 January). UNSOM Congratulates President Said Abdullahi Deni on Election as President of Puntland. Retrieved 16 March 2021, from https://unsom.unmissions.org/unsomcongratulates-said-abdullahi-deni-election-president-puntland-0

8 OCHA. (2020). Somalia: Situation Report, 10 December 2020. Retrieved 16 March 2021, from https://reports.unocha.org/en/country/somalia

9 Thomson Reuters Foundation. (n.d.) The World's Most Dangerous Countries for Women 2018: Somalia Country Summary. Retrieved 16 March 2021, from https://poll2018.trust.org/country/?id=somalia

10 Fanning, E. (2018). Drought, Displacement and Livelihoods in Somalia/Somaliland: Time for GenderSensitive and Protection-Focused Approaches. Concern Worldwide, Danish Refugee Council, Norwegian Refugee Council, Plan International, REACH, Save Somali Women and Children, Oxfam. Retrieved 16 March 2021, from https://reliefweb.int/sites/reliefweb.int/files/resources/bn-somaliadrought-displacement-protection-gender-250618-en.pdf

11 PLHDS. (2021). Puntland Report 2020: Somali Health and Demographic Survey. Retrieved 16 March 2021, from https://somalia.unfpa.org/sites/default/files/pubpdf/puntland shds report 2020 v15 final.pdf

12 Ministry of Planning, Economic Development and International Cooperation. (2020, 29 November). Puntland Launches its First Health and Demographic Survey. Retrieved 16 March 2021, from https://pl.statistics.so/puntland-launches-its-first-health-and-demographic-survey

13 For more information, see http://www.kaalo.org/who-we-are/about-us

14 Adeso Africa, formerly known as Horn Relief, is a humanitarian and development non-profit organization. https://adesoafrica.org/

15 Both words mean 'support' in the Somali language, in this context referring to the support people get from their relatives and close networks.

16 A secret and informal type of marriage.

17 See https://moh.nomadilab.org data for 16 March 2021.

18 The biggest telecommunications company in Puntland. 


\section{ACKNOWLEDGEMENTS}

This report was written by Kasey Ochiltree (Humanitarian Gender Adviser, Oxfam America) and lulia Andreea Toma (Gender Adviser, GHT), with research conducted and support provided by Muna Hussein (Gender Officer, Somaliland); Abdirashid Yousuf (Gender and Protection Coordinator, Somaliland); and Mohamud Birik Adan (Protection Manager, KAALO - partner organization).

This research would not have been possible without funding from GFFO, without the support provided by our partner organization KAALO, and more importantly, without the involvement of community men, women, girls and boys in the Puntland locations, as well as staff from key agencies, who all generously gave their time in the survey, focus group discussions and key informant interviews on which this analysis is based.

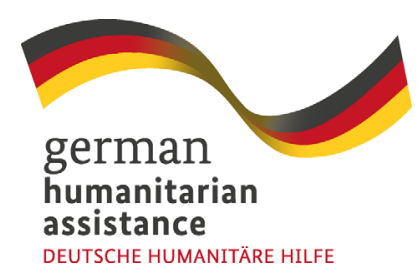




\section{Research reports}

This research report was written to share research results, to contribute to public debate and to invite feedback on development and humanitarian policy and practice. It does not necessarily reflect the policy positions of the publishing organizations. The views expressed are those of the author and not necessarily those of the publishers.

For more information, or to comment on this report, email Kasey Ochiltree (kasey.ochiltree@oxfam.org) and Iulia Andreea Toma (iulia.toma@oxfam.org).

(C) Oxfam International and KAALO April 2021

This publication is copyright but the text may be used free of charge for the purposes of advocacy, campaigning, education, and research, provided that the source is acknowledged in full. The copyright holder requests that all such use be registered with them for impact assessment purposes. For copying in any other circumstances, or for re-use in other publications, or for translation or adaptation, permission must be secured and a fee may be charged. Email policyandpractice@oxfam.org.uk.

The information in this publication is correct at the time of going to press.

Published by Oxfam GB for Oxfam International and KAALO under ISBN 978-1-78748-748-2 in April 2021. DOI: $10.21201 / 2021.7482$

Oxfam GB, Oxfam House, John Smith Drive, Cowley, Oxford, OX4 2JY, UK.

\section{OXFAM}

Oxfam is an international confederation of 21 organizations networked together in 67 countries, as part of a global movement for change, to build a future free from the injustice of poverty. Please write to any of the agencies for further information, or visit www.oxfam.org.

\section{KAALO}

KAALO Aid and Development (KAALO) was established in March 1991 just after the collapse of Somali central government. It is one of the most successful and consistent community based, non-profit, humanitarian and development NGOs in Somalia. KAALO operates in the Puntland Regions, and Somalia at large and is involved in relief aid, rehabilitation, and development programs in various sectors. http://www.kaalo.org/. 